\title{
A two-gradient approach for phase transitions in thin films
}

\author{
Bernardo Galvão-Sousa and Vincent Millot
}

\begin{abstract}
Motivated by solid-solid phase transitions in elastic thin films, we perform a $\Gamma$-convergence analysis for a singularly perturbed energy related to second order phase transitions in a domain of vanishing thickness. Under a two-wells assumption, we derive a sharp interface model with an interfacial energy depending on the asymptotic ratio between the characteristic length scale of the phase transition and the thickness of the film. In each case, the interfacial energy is determined by an explicit optimal profile problem. This asymptotic problem entails a nontrivial dependance on the thickness direction when the phase transition is created at the same rate as the thin film, while it shows a separation of scales if the thin film is created at a faster rate than the phase transition. The last regime, when the phase transition is created at a faster rate than the thin film, is more involved. Depending on growth conditions of the potential and the compatibility of the two phases, we either obtain a sharp interface model with scale separation, or a trivial situation driven by rigidity effects.
\end{abstract}

Mathematics Subject Classification (2000). 35G99, 49J40, 49J45, 49K20, 74K35, 74N99.

Keywords. Phase transitions, Thin films, Singular perturbations, Double-well potential, $\Gamma$-convergence.

\section{Introduction}

In the last few years, many mathematical efforts have been devoted to variational problems arising in the modelling of phase transitions in solids, see e.g. $[6,16-18]$. These problems often involve singularly perturbed functionals of the form

$$
\mathbf{E}_{\varepsilon}(\mathbf{u}, \mathscr{B})=\int_{\mathscr{B}} \frac{1}{\varepsilon} W(\nabla \mathbf{u})+\varepsilon\left|\nabla^{2} \mathbf{u}\right|^{2} d \mathbf{x},
$$


where $\mathbf{u}: \mathscr{B} \subset \mathbb{R}^{3} \rightarrow \mathbb{R}^{3}$ represents the displacement of an elastic body $\mathscr{B}, \varepsilon>0$ is a small parameter, and $W$ is a (nonnegative) free energy density with multiple minima corresponding to martensitic materials. Due to the multiple well structure, nucleation of phases in a given configuration may occur without increasing $\int W(\nabla \mathbf{u})$, so that the free energy may admit many (eventually constrained) minimizers. In order to select preferred configurations, the Van der Waals-Cahn-Hilliard theory adds higher order terms leading to functionals of the form (1.1). In such functionals a competition occurs between the two terms: the free energy favors gradients close to a minimum value of $W$, while $\left|\nabla^{2} \mathbf{u}\right|^{2}$ penalizes transitions from one minima to another.

The $\Gamma$-convergence method provides a suitable framework to study the asymptotic behavior of singularly perturbed energies like $\mathbf{E}_{\varepsilon}$ (see e.g. [12,19] for a more detailed overview of this subject). One of the first applications of $\Gamma$-convergence was actually obtained in $[30,31,35]$ in the context of fluid-fluid phase transitions (see e.g. [25]). Here the authors deal with energy functionals of the form $\int \frac{1}{\varepsilon} W(v)+\varepsilon|\nabla v|^{2} d x$ where the potential $W$ has a double well structure, i.e., $\{W=0\}=\{\alpha, \beta\}$. It is shown that such family of energies $\Gamma$-converges (in a suitable topology) as $\varepsilon \rightarrow 0$ to a functional which calculates the area of the interface between the two phases $\alpha$ and $\beta$, for limiting $B V$ functions $v$ with values in $\{\alpha, \beta\}$. Since then this result has been generalized in many different ways (see e.g. $[1,5,7,10,24,32]$ ), in particular in [23] for an intermediate situation where the singular perturbation $|\nabla v|^{2}$ is replaced by the higher order term $\left|\nabla^{2} v\right|^{2}$. The first $\Gamma$-convergence result for functionals acting on gradient vector fields has been obtained in [16]. Assuming that $\{W=0\}=\{A, B\}$ for some rank-one connected matrices $A$ and $B$ (and some additional constitutive conditions on $W$ ), the authors prove the $\Gamma$-convergence of $\mathbf{E}_{\varepsilon}$ as $\varepsilon \rightarrow 0$. Once again the effective functional returns the total area of the interfaces separating the phases $A$ and $B$, for limiting functions $\mathbf{u}$ satisfying $\nabla \mathbf{u} \in\{A, B\}$ a.e. and $\nabla \mathbf{u} \in B V$. Here the rank-one connection between the wells $A$ and $B$ turns out to be necessary for the existence of non-affine u's satisfying $\nabla \mathbf{u} \in\{A, B\}$, and the interfaces must be planar and oriented according to the connection, see [6]. We also mention recent developments on weakening the condition on the wells of $W$ to allow for frame indifference, i.e., assuming the zero level set of $W$ of the form $S O(3) A \cup S O(3) B$ (see $[17,18]$ ).

Another topic of increasing interest related to solid mechanics concerns thin elastic films. It is well known that thin films may have different mechanical properties from bulk materials, specifically for martensitic ones. Those properties are important for many physical applications (see [9]). In this context, the $\Gamma$-convergence point of view is again suitable to rigorously derive limiting models starting from 3D nonlinear elasticity. This has been shown in $[28,13]$ for the membrane theory, and more recently in $[21,22]$ for nonlinear plate models. In the regime of membranes, several studies have focused their attention on the impact of a higher order perturbation on the behavior of thin films. The first variational approach has been addressed in [9] where the authors add the singular perturbation $\varepsilon^{2} \int\left|\nabla^{2} \mathbf{u}\right|^{2}$ to the free energy $\int W(\nabla \mathbf{u})$ for a domain of small thickness $h$. They obtain in the limit $h \rightarrow 0$ a $2 \mathrm{D}$ energy density which 
depends on the deformation gradient of the mid-surface, and the Cosserat vector $b$ which gives an asymptotic description of the out-of-plane deformation. An important consequence of the results of [9] is that for many interesting materials the low energy states in the thin film limit are indeed different from the ones in three dimensional samples. However [9] does not treat possible correlations between the thickness $h$ and the parameter $\varepsilon$. This issue was first conducted in [34], and more intensively in [20] to keep track of the Cosserat vector. It is shown in [20] that the limiting model is determined by the asymptotic ratio $h / \varepsilon$ as $h \rightarrow 0$ and $\varepsilon \rightarrow 0$, and it depends whether $h / \varepsilon \sim 0, h / \varepsilon \sim \infty$, or $h / \varepsilon \sim 1$.

The general idea of this paper is to study a simple class of singularly perturbed functionals related to phase transitions in thin films. In this direction, some models have been recently analyzed, see [8], and also [15,26] for models without singular perturbation leading to sharp interfaces. Here we want to carry out an analysis in the spirit of [16] focusing on possible correlations between the strength of an interfacial energy and the thickness of the film. As in $[9,20,34]$ we consider a "membrane scaling", and we introduce the normalized functional $\mathbf{F}_{\varepsilon}^{h}$ defined for $\mathbf{u} \in H^{2}\left(\Omega_{h} ; \mathbb{R}^{3}\right)$ by

$$
\mathbf{F}_{\varepsilon}^{h}(\mathbf{u}):=\frac{1}{h} \int_{\Omega_{h}} W(\nabla \mathbf{u})+\varepsilon^{2}\left|\nabla^{2} \mathbf{u}\right|^{2} d \mathbf{x},
$$

where $\Omega_{h}:=\omega \times h I \subset \mathbb{R}^{3}, I:=\left(-\frac{1}{2}, \frac{1}{2}\right)$, and the mid-surface $\omega \subset \mathbb{R}^{2}$ is a bounded convex open set (here convexity is assumed for simplicity, and we refer to [16] for more general geometries). Considering configurations $\mathbf{u}$ with energy of order $\varepsilon$, that is $\mathbf{F}_{\varepsilon}^{h}(\mathbf{u}) \leqslant O(\varepsilon)$, and renormalizing by $1 / \varepsilon$ we are led to the energy $\frac{1}{h} \mathbf{E}_{\varepsilon}$ in the thin domain $\Omega_{h}$. We are interested in the variational convergence of the family $\left\{\frac{1}{h} \mathbf{E}_{\varepsilon}\left(\cdot, \Omega_{h}\right)\right\}$ as $h \rightarrow 0$ and $\varepsilon \rightarrow 0$. To this aim, we introduce the standard rescaling

$$
u(x)=\mathbf{u}(\mathbf{x}) \text { with }\left(x_{1}, x_{2}, x_{3}\right)=\left(\mathbf{x}_{1}, \mathbf{x}_{2}, \frac{\mathbf{x}_{3}}{h}\right),
$$

which yields functionals $\left\{F_{\varepsilon}^{h}\right\}$ defined for $u \in H^{2}\left(\Omega ; \mathbb{R}^{3}\right)$ by

$$
F_{\varepsilon}^{h}(u):=\int_{\Omega} \frac{1}{\varepsilon} W\left(\nabla_{h} u\right)+\varepsilon\left|\nabla_{h}^{2} u\right|^{2} d x,
$$

where $\Omega:=\Omega_{1}$, and $\nabla_{h}:=\left(\partial_{1}, \partial_{2}, \frac{1}{h} \partial_{3}\right)$ is the rescaled gradient operator.

Our main goal is to perform the $\Gamma$-convergence as $\varepsilon \rightarrow 0$ and $h \rightarrow 0$ of the family $\left\{F_{\varepsilon}^{h}\right\}$ in the simplest context where we can illustrate a difference between the behavior of thin films and bulk materials. The class of "toy models" we have in mind involves double-well potentials $W$ of the type considered in [16]. In other words, $W$ should be structurally similar to

$$
W(\xi) \approx \operatorname{dist}(\xi,\{A, B\})^{p}=\min \left\{|\xi-A|^{p},|\xi-B|^{p}\right\} .
$$

A situation where a qualitatively different behavior from [16] is expected is when $A$ and $B$ are not rank-one connected, but $A^{\prime}$ and $B^{\prime}$ are. Here we denote by $A^{\prime}$ and $B^{\prime}$ the $3 \times 2$ matrices extracted by taking the first two columns from $A$ and $B$, respectively. In this case, sequences with bounded $\mathbf{E}_{\varepsilon}$ energy, 
as in [16], converge to affine maps by the results in [6]. But, as it will be made precise below, this rigidity effect might not occur for sequences with bounded $F_{\varepsilon}^{h}$ energy since $A^{\prime}$ and $B^{\prime}$ are compatible on the mid-surface. Accordingly, we assume that $W: \mathbb{R}^{3 \times 3} \rightarrow[0, \infty)$ is continuous and satisfies the following first set of assumptions:

$\left(H_{1}\right)\{W=0\}=\{A, B\}$ where $A=\left(A^{\prime}, A_{3}\right) \in \mathbb{R}^{3 \times 2} \times \mathbb{R}^{3}$ and $B=\left(B^{\prime}, B_{3}\right) \in$ $\mathbb{R}^{3 \times 2} \times \mathbb{R}^{3}$ are distinct matrices satisfying $A^{\prime}-B^{\prime}=2 a \otimes \bar{\nu}$ for some $a \in \mathbb{R}^{3}$ and $\bar{\nu} \in \mathbb{S}^{1}$

$\left(H_{2}\right) \frac{1}{C_{1}}|\xi|^{p}-C_{1} \leqslant W(\xi) \leqslant C_{1}|\xi|^{p}+C_{1}$, for $p \geqslant 2$ and some constant $C_{1}>0$.

Under the conditions $\left(H_{1}\right)$ and $\left(H_{2}\right)$, we shall derive compactness properties for sequences with uniformly bounded energy. In our setting the limiting configurations space turns out to be

$$
\begin{gathered}
\mathscr{C}:=\left\{(u, b) \in W^{1, \infty}\left(\Omega ; \mathbb{R}^{3}\right) \times L^{\infty}\left(\Omega ; \mathbb{R}^{3}\right):\left(\nabla^{\prime} u, b\right) \in B V(\Omega ;\{A, B\}),\right. \\
\left.\partial_{3} u=\partial_{3} b=0\right\},
\end{gathered}
$$

where we write $\nabla^{\prime}:=\left(\partial_{1}, \partial_{2}\right)$. Throughout the paper we identify pairs $(u, b) \in$ $\mathscr{C}$ with functions defined on the mid-surface $\omega$, that is $u(x)=u\left(x^{\prime}\right), b(x)=$ $b\left(x^{\prime}\right)$ with $x^{\prime}:=\left(x_{1}, x_{2}\right)$. In particular, for any $(u, b) \in \mathscr{C}$, we can write

$$
\left(\nabla^{\prime} u, b\right)\left(x^{\prime}\right)=\left(1-\chi_{E}\left(x^{\prime}\right)\right) A+\chi_{E}\left(x^{\prime}\right) B \quad \text { for } \mathcal{L}^{2} \text {-a.e. } x^{\prime} \in \omega,
$$

where $E \subset \omega$ is a set of finite perimeter in $\omega$, and $\chi_{E}$ denotes its characteristic function. For $A^{\prime} \neq B^{\prime}$ the (reduced) boundary of $E$ consists of countably many planar interfaces with normal $\bar{\nu}$, while $E$ is an arbitrary set of finite perimeter in $\omega$ if $A^{\prime}=B^{\prime}$ (see Theorem 2.1, and [6]). Let us recall that, in our setting, $A$ and $B$ might not be rank-one connected, so that we shall have to construct recovery sequences substantially different from [16]. We also emphasize that for $A^{\prime}=B^{\prime}$, the arbitrary geometry of the interface is again in sharp contrast with [16], where interfaces must be made by hyperplanes.

The general compactness result is formulated in Theorem 1.1 below. As a matter of fact this theorem does not provide optimal compactness (only) in the case $\varepsilon \ll h$. Indeed, in this regime we may expect a separation of scales to hold and the film to behave like a three dimensional sample. Thus, if the wells are not compatible in the bulk, i.e., $\operatorname{rank}(A-B)>1$, it is reasonable to believe that sequences with bounded energy should converge to trivial limits. This question will be addressed in the last section with positive results for some particular cases (see Theorems 6.9 and 6.10).

Theorem 1.1. (Compactness) Assume that $\left(H_{1}\right)-\left(H_{2}\right)$ hold. Let $h_{n} \rightarrow 0^{+}$ and $\varepsilon_{n} \rightarrow 0^{+}$be arbitrary sequences, and let $\left\{u_{n}\right\} \subset H^{2}\left(\Omega ; \mathbb{R}^{3}\right)$ be such that $\sup _{n} F_{\varepsilon_{n}}^{h_{n}}\left(u_{n}\right)<\infty$. Then there exist a subsequence (not relabeled) and $(u, b) \in$ $\mathscr{C}$ such that $u_{n}-f_{\Omega} u_{n} d x \rightarrow u$ in $W^{1, p}\left(\Omega ; \mathbb{R}^{3}\right)$ and $\frac{1}{h_{n}} \partial_{3} u_{n} \rightarrow b$ in $L^{p}\left(\Omega ; \mathbb{R}^{3}\right)$.

To describe our $\Gamma$-convergence results we need some additional assumptions on the potential $W$ (these assumptions will be used only in the construction of recovery sequences). First of all we assume, without loss of generality, that $A=-B$ and $\bar{\nu}=e_{1}^{\prime}:=(1,0)$, so that 


$$
A^{\prime}=-B^{\prime}=a \otimes e_{1}^{\prime}, \quad A_{2}=B_{2}=0, \quad \text { and } \quad A_{3}=-B_{3} .
$$

Indeed, the general case can be reduced to (1.5) by considering a modified bulk energy density $W_{\text {mod }}$ defined by $W_{\bmod }(\xi):=W(\xi R+C)$ where $C=1 / 2(A+B)$ and $R=\operatorname{diag}\left(R^{\prime}, 1\right)$ with $R^{\prime} \in S O(2)$ satisfying $R^{\prime} \bar{\nu}=e_{1}^{\prime}$. This new potential $W_{\text {mod }}$ obviously satisfies hypotheses $\left(H_{1}\right)$ and $\left(H_{2}\right)$ with $(1.5)$. Our second set of assumptions requires $W$ to share some structural properties of the prototypical function defined in (1.2). More precisely, given (1.5), we assume that $\left(H_{3}\right)$ there exist constants $\varrho>0$ and $C_{2}>0$ such that

$$
\frac{1}{C_{2}} \operatorname{dist}(\xi,\{A, B\})^{p} \leqslant W(\xi) \leqslant C_{2} \operatorname{dist}(\xi,\{A, B\})^{p} \text { if } \operatorname{dist}(\xi,\{A, B\}) \leqslant \varrho ;
$$

$\left(H_{4}\right) W\left(\xi_{1}, 0, \xi_{3}\right) \leqslant W(\xi)$ for all $\xi=\left(\xi_{1}, \xi_{2}, \xi_{3}\right) \in \mathbb{R}^{3 \times 3}$;

$\left(H_{5}\right)$ if $A^{\prime}=B^{\prime}=0$, then $W\left(\xi^{\prime}, \xi_{3}\right)=V\left(\left|\xi^{\prime}\right|, \xi_{3}\right)$ for some $V:[0,+\infty) \times \mathbb{R}^{3} \rightarrow$ $[0, \infty)$.

Here $|\cdot|$ stands for the usual Euclidean norm, $\xi^{\prime}:=\left(\xi_{1}, \xi_{2}\right) \in \mathbb{R}^{3 \times 2}$ and $\left|\xi^{\prime}\right|^{2}=\left|\xi_{1}\right|^{2}+\left|\xi_{2}\right|^{2}$. Observe that in the case $A^{\prime}=B^{\prime}$ (so that (1.5) yields $\left.A^{\prime}=B^{\prime}=0\right)$, assumptions $\left(H_{4}\right)$ and $\left(H_{5}\right)$ require the function $r \mapsto V(r, z)$ to be nondecreasing for every $z \in \mathbb{R}^{3}$. Taking (1.5) into account, we also notice that these assumptions are clearly satisfied for $W(\xi)=\operatorname{dist}(\xi,\{A, B\})^{p}$ with $V(r, z):=\left(r^{2}+\operatorname{dist}^{2}\left(z,\left\{A_{3}, B_{3}\right\}\right)\right)^{p / 2}$. Let us finally mention that similar assumptions are already present in [16]. Condition $\left(H_{3}\right)$ is a standard non-degeneracy condition on $W$ near the wells, while $\left(H_{4}\right)$ allows one to construct lower dimensional optimal profiles connecting the two phases $A$ and $B$. Hypothesis $\left(H_{5}\right)$ is a more technical isotropy condition, that we assume for simplicity.

Let us now consider the family of functionals $\mathcal{F}_{\varepsilon}^{h}:\left[L^{1}\left(\Omega ; \mathbb{R}^{3}\right)\right]^{2} \rightarrow[0, \infty]$ defined by

$$
\mathcal{F}_{\varepsilon}^{h}(u, b):= \begin{cases}F_{\varepsilon}^{h}(u) & \text { if } u \in H^{2}\left(\Omega ; \mathbb{R}^{3}\right) \text { and } b=\frac{1}{h} \partial_{3} u, \\ +\infty & \text { otherwise. }\end{cases}
$$

We will prove that the behavior of $\mathcal{F}_{\varepsilon}^{h}$ depends, as expected, on the asymptotic ratio $\frac{h}{\varepsilon} \rightarrow \gamma \in[0, \infty]$ as $h$ and $\varepsilon$ tend to 0 , and that the family $\left\{\mathcal{F}_{\varepsilon}^{h}\right\}$ $\Gamma$-converges to a functional $\mathscr{F}_{\gamma}:\left[L^{1}\left(\Omega ; \mathbb{R}^{3}\right)\right]^{2} \rightarrow[0, \infty]$ given by

$$
\mathscr{F}_{\gamma}(u, b):= \begin{cases}K_{\gamma} \operatorname{Per}_{\omega}(E) & \text { if }(u, b) \in \mathscr{C}, \\ +\infty & \text { otherwise }\end{cases}
$$

where $\left(\nabla^{\prime} u, b\right)(x)=\left(1-\chi_{E}\left(x^{\prime}\right)\right) A+\chi_{E}\left(x^{\prime}\right) B$ as in (1.4), and $\operatorname{Per}_{\omega}(E):=$ $\mathcal{H}^{1}\left(\partial^{*} E \cap \omega\right)$ denotes the (measure theoretic) perimeter of $E$ in $\omega$. Here the constant $K_{\gamma}>0$ is determined by an optimal profile problem for connecting phase $A$ to phase $B$. By assumption $\left(H_{4}\right)$, we will be able to describe $K_{\gamma}$ through a lower dimensional variational problem. To simplify the notation, we introduce the $2 \mathrm{D}$ energy density $\mathcal{W}: \mathbb{R}^{3 \times 2} \rightarrow[0, \infty)$ given by

$$
\mathcal{W}\left(\zeta_{1}, \zeta_{2}\right):=W\left(\zeta_{1}, 0, \zeta_{2}\right)
$$

which is a double-well potential with zero level set $\left\{\left(A_{1}, A_{3}\right),\left(B_{1}, B_{3}\right)\right\}$. 
Our first convergence result deals with the critical regime where the thickness of the film and the strength of the interfacial energy are of the same order, that is $\gamma \in(0, \infty)$.

Theorem 1.2. (Critical Regime) Assume that $\left(H_{1}\right)-\left(H_{5}\right)$ hold with (1.5). Let $h_{n} \rightarrow 0^{+}$and $\varepsilon_{n} \rightarrow 0^{+}$be arbitrary sequences such that $h_{n} / \varepsilon_{n} \rightarrow \gamma$ for some $\gamma \in(0, \infty)$. Then the functionals $\left\{\mathcal{F}_{\varepsilon_{n}}^{h_{n}}\right\} \Gamma$-converge for the strong $L^{1}$-topology to the functional $\mathscr{F}_{\gamma}$ given by (1.6) with

$$
K_{\gamma}:=\inf \left\{\frac{1}{\gamma} \int_{\ell I \times \gamma I} \mathcal{W}(\nabla v)+\left|\nabla^{2} v\right|^{2} d y: \ell>0, v \in C^{2}\left(\ell I \times \gamma I ; \mathbb{R}^{3}\right),\right.
$$

$\nabla v(y)=\left(A_{1}, A_{3}\right)$ nearby $\left\{y_{1}=\ell / 2\right\}$ and $\nabla v(y)=\left(B_{1}, B_{3}\right)$ nearby $\left.\left\{y_{1}=-\ell / 2\right\}\right\}$.

We observe that the formula for $K_{\gamma}$ (with $\gamma \in(0, \infty)$ ) entails a highly nontrivial dependence on the vertical direction in the asymptotic problem. In fact, in the case $A_{3}=B_{3}$, one can find potentials $W$ for which a nontrivial dependance on $x_{3}$ still occurs, see [16, Section 8]. Note that in many second order phase transitions problems, optimal profiles usually have an oscillatory behavior along the limiting interface, see $[16,27]$ and references therein (see also Theorem 1.4 below).

In contrast with the critical regime, one may expect the case $\gamma=0$ (i.e., $h \ll \varepsilon)$ to lead to a simpler behavior with respect to the $x_{3}$-variable by separation of scales. Indeed, the energies formally behave like two dimensional ones, and optimal transition layers should only depend on the distance to the interface by assumptions $\left(H_{4}\right)-\left(H_{5}\right)$. We will illustrate this fact with more details in Sect. 5 (see Remark 5.9). Our results for this regime give a positive answer to our formal discussion, and they can be summarized in the following theorem.

Theorem 1.3. (Subcritical Regime) Assume that $\left(H_{1}\right)-\left(H_{5}\right)$ hold with (1.5). Let $h_{n} \rightarrow 0^{+}$and $\varepsilon_{n} \rightarrow 0^{+}$be arbitrary sequences such that $h_{n} / \varepsilon_{n} \rightarrow 0$. Then the functionals $\left\{\mathcal{F}_{\varepsilon_{n}}^{h_{n}}\right\} \Gamma$-converge for the strong $L^{1}$-topology to the functional $\mathscr{F}_{0}$ given by (1.6) with

$$
\begin{array}{r}
K_{0}:=\inf \left\{\int_{-\ell}^{\ell} \mathcal{W}(\phi(t))+\left|\phi_{1}^{\prime}(t)\right|^{2}+2\left|\phi_{2}^{\prime}(t)\right|^{2} d t: \ell>0, \phi=\left(\phi_{1}, \phi_{2}\right)\right. \\
\left.\quad \in C^{1}\left([-\ell, \ell] ; \mathbb{R}^{3 \times 2}\right), \phi(\ell)=\left(A_{1}, A_{3}\right) \text { and } \phi(-\ell)=\left(B_{1}, B_{3}\right)\right\} .
\end{array}
$$

In the supercritical case $\gamma=+\infty$ (i.e., $\varepsilon \ll h$ ), one may again expect a separation of scales to hold. In other words, we should be able to recover the limiting functional by taking first the limit $\varepsilon \rightarrow 0$, and then the thin film limit $h \rightarrow 0$. Hence, to obtain a nontrivial $\Gamma$-limit, it is natural to ask for $A$ and $B$ to be compatible in the bulk with a non vertical connection (see (1.9) below). As already mentioned, we have exhibited rigidity effects in the other cases, 
at least for some particular potentials (see Theorems 6.9 and 6.10). For this reason we assume in the supercritical regime that $A-B$ is a rank-one matrix, and that $A^{\prime} \neq B^{\prime}$. Under the structure (1.5), this assumption is equivalent to the existence of $\lambda \in \mathbb{R}$ such that $A_{3}=-B_{3}=\lambda a$. Then the wells $A$ and $B$ can be written as

$$
A=-B=a \otimes\left(e_{1}+\lambda e_{3}\right) \quad(a \neq 0) .
$$

We have obtained partial results for this regime through lower and upper bounds for the $\Gamma$-lim inf and $\Gamma$-lim sup, respectively. Fortunately, our estimates turn out to be nearly optimal in the sense that upper and lower bounds agree whenever $\lambda=0, p=2$, and $W$ is symmetric with respect to $\xi_{3}$ (which is the case for the potential (1.2) assuming (1.9)). In this latter case, it follows that the separation of scales is indeed true by [16, Theorem 1.4] (see Remark 6.8).

Theorem 1.4. (Supercritical Regime) Assume that $\left(H_{1}\right)-\left(H_{4}\right)$ and (1.9) hold for some $\lambda \in \mathbb{R}$. Let $h_{n} \rightarrow 0^{+}$and $\varepsilon_{n} \rightarrow 0^{+}$be arbitrary sequences such that $h_{n} / \varepsilon_{n} \rightarrow \infty$. Then,

$$
c \mathscr{F}_{\infty} \leqslant \Gamma\left(L^{1}\right)-\liminf _{n \rightarrow+\infty} \mathcal{F}_{\varepsilon_{n}}^{h_{n}} \quad \text { and } \quad \Gamma\left(L^{1}\right)-\limsup _{n \rightarrow+\infty} \mathcal{F}_{\varepsilon_{n}}^{h_{n}} \leqslant \mathscr{F}_{\infty},
$$

for a constant $c>0$, where the functional $\mathscr{F}_{\infty}$ is given by (1.6) with

$$
\begin{aligned}
& K_{\infty}:=\left(1+\lambda^{2}\right)^{\frac{1}{2}} \inf \left\{\int_{Q_{\lambda}^{\prime}} \ell \mathcal{W}(\nabla v)+\frac{1}{\ell}\left|\nabla^{2} v\right|^{2} d y: \ell>0, v \in C^{2}\left(\mathbb{R}^{2} ; \mathbb{R}^{3}\right),\right. \\
& \quad \nabla v(y)=\left(A_{1}, A_{3}\right) \text { nearby }\left\{y \cdot \nu_{\lambda}=\ell / 2\right\}, \nabla v(y)=\left(B_{1}, B_{3}\right) \text { nearby }\left\{y \cdot \nu_{\lambda}=-\ell / 2\right\}, \\
& \text { and } \left.v \text { is } 1 \text { - periodic in the direction orthogonal to } \nu_{\lambda}\right\},
\end{aligned}
$$

where $\nu_{\lambda}:=\frac{1}{\sqrt{1+\lambda^{2}}}(1, \lambda) \in \mathbb{S}^{1}$, and $Q_{\lambda}^{\prime}$ denotes the unit cube of $\mathbb{R}^{2}$ centered at the origin with two faces orthogonal to $\nu_{\lambda}$. Moreover, if $p=2$ in $\left(H_{1}\right), \lambda=0$ in (1.9), and $W$ satisfies $W\left(\xi^{\prime}, \xi_{3}\right)=W\left(\xi^{\prime},-\xi_{3}\right)$ for all $\xi \in \mathbb{R}^{3 \times 3}$, then the functionals $\left\{\mathcal{F}_{\varepsilon_{n}}^{h_{n}}\right\} \Gamma$-converge for the strong $L^{1}$-topology to $\mathscr{F}_{\infty}$.

The paper is organized as follows. We start in Sect. 2 with a structure result for the class $\mathscr{C}$ of limiting maps, and the compactness theorem is proved in Sect. 3. The proofs of Theorems 1.2, 1.3, and 1.4 are given in Sects. 4, 5, and 6 respectively. We complete Sect. 6 with the aforementioned rigidity results in the supercritical case.

\section{Preliminaries}

Throughout the paper, $Q$ and $Q^{\prime}$ denote the standard open unit cubes centered at the origin of $\mathbb{R}^{3}$ and $\mathbb{R}^{2}$ respectively, while $I:=\left(-\frac{1}{2}, \frac{1}{2}\right)$. For simplicity, the differential operators $\frac{\partial}{\partial x_{i}}$ and $\frac{\partial^{2}}{\partial x_{i} \partial x_{j}}$ are written $\partial_{i}$ and $\partial_{i j}^{2}$ respectively. The rescaled gradient $\nabla_{h}$ and rescaled Hessian $\nabla_{h}^{2}$ operators are given by

$$
\nabla_{h} u=\left(\partial_{1} u, \partial_{2} u, \frac{1}{h} \partial_{3} u\right) \quad \text { and } \quad \nabla_{h}^{2} u=\left(\begin{array}{lll}
\partial_{11}^{2} u & \partial_{12}^{2} u & \frac{1}{h} \partial_{13}^{2} u \\
\partial_{12}^{2} u & \partial_{22}^{2} u & \frac{1}{h} \partial_{23}^{2} u \\
\frac{1}{h} \partial_{13}^{2} u & \frac{1}{h} \partial_{23}^{2} u & \frac{1}{h^{2}} \partial_{33}^{2} u
\end{array}\right)
$$


For a Borel set $B \subset \mathbb{R}^{3}$ and an admissible map $u$ we write

$$
F_{\varepsilon}^{h}(u, B):=\int_{B} \frac{1}{\varepsilon} W\left(\nabla_{h} u\right)+\varepsilon\left|\nabla_{h}^{2} u\right|^{2} d x,
$$

In the sequel, it will be useful to consider the two reference maps, $u_{0}$ and $b_{0}$, defined for $x \in \mathbb{R}^{3}$ by

$$
u_{0}(x):=\bar{u}_{0}\left(x_{1}\right) \quad \text { and } \quad b_{0}(x):=\bar{b}_{0}\left(x_{1}\right),
$$

where $\bar{u}_{0}$ and $\bar{b}_{0}$ are given by

$$
\bar{u}_{0}(t):=|t| a \text { and } \quad \bar{b}_{0}(t):= \begin{cases}A_{3} & \text { if } t \geqslant 0 \\ B_{3} & \text { if } t<0 .\end{cases}
$$

We shall follow [4] for the standard results and notations on functions of bounded variation. We only recall that, given an open set $\mathscr{O} \subset \mathbb{R}^{N}$, a Borel set $E \subset \mathscr{O}$ is said to be of finite perimeter in $\mathscr{O}$ if its characteristic function $\chi_{E}$ belongs to $B V(\mathscr{O})$. In such a case, the perimeter of $E$ in $\mathscr{O}$, that we write $\operatorname{Per}_{\mathscr{O}}(E)$, is the total variation $\left|D \chi_{E}\right|(\mathscr{O})$, and it is equal to $\mathcal{H}^{N-1}\left(\partial^{*} E \cap \mathscr{O}\right)$ where $\partial^{*} E$ denotes the reduced boundary of $E$, and $\mathcal{H}^{N-1}$ is the $(N-1)$ dimensional Hausdorff measure.

We now state a structure result for the class $\mathscr{C}$ of limiting configurations [see (1.3)]. To this purpose, let us define

$$
\begin{gathered}
\alpha_{\min }:=\inf \left\{x_{1} \in \mathbb{R}: x=\left(x_{1}, x_{2}\right) \in \omega\right\} \quad \text { and } \\
\alpha_{\max }:=\sup \left\{x_{1} \in \mathbb{R}: x=\left(x_{1}, x_{2}\right) \in \omega\right\} .
\end{gathered}
$$

We have the following theorem as a consequence of $[6,16]$.

Theorem 2.1. Assume that (1.5) holds. Then for every pair $(u, b) \in \mathscr{C},\left(\nabla^{\prime} u, b\right)$ is of the form

$$
\left(\nabla^{\prime} u(x), b(x)\right)=\left(1-\chi_{E}\left(x^{\prime}\right)\right) A+\chi_{E}\left(x^{\prime}\right) B,
$$

where $E \subset \omega$ is a set of finite perimeter in $\omega$. Moreover, if $A^{\prime} \neq B^{\prime}$ then $u$ is of the form

$$
u(x)=c_{0}+x_{1} a-2 \psi\left(x_{1}\right) a,
$$

where $c_{0} \in \mathbb{R}^{3}, c_{0} \cdot a=0, \psi \in W^{1, \infty}\left(\left(\alpha_{\min }, \alpha_{\max }\right) ; \mathbb{R}\right)$ and $\psi^{\prime} \in B V_{\mathrm{loc}}$ $\left(\left(\alpha_{\min }, \alpha_{\max }\right) ;\{0,1\}\right)$. In particular, if $A^{\prime} \neq B^{\prime}$ then $E$ is layered perpendicularly to $e_{1}^{\prime}$, i.e.,

$$
\partial^{*} E \cap \omega=\bigcup_{i \in \mathscr{I}}\left\{\alpha_{i}\right\} \times J_{i},
$$

where $\mathscr{I} \subset \mathbb{Z}$ is made by successive integers, $\left\{\alpha_{i}\right\} \subset\left(\alpha_{\min }, \alpha_{\max }\right)$ is locally finite in $\left(\alpha_{\min }, \alpha_{\max }\right), \alpha_{i}<\alpha_{i+1}$, and the sets $J_{i}:=\left\{t \in \mathbb{R}:\left(\alpha_{i}, t\right) \in \omega\right\}$ are open bounded intervals.

Proof. Step 1. We start with the case where $A^{\prime} \neq B^{\prime}$. Given $(u, b) \in \mathscr{C}$, we can write

$$
\left(\nabla^{\prime} u, b\right)=\left(1-\chi_{F}\right) A+\chi_{F} B
$$


for some set $F \subset \Omega$ of finite perimeter in $\Omega$. Since $\partial_{3} u=0$, we have $\nabla u \in$ $B V\left(\Omega ;\left\{\left(A^{\prime}, 0\right),\left(B^{\prime}, 0\right)\right\}\right)$. Then we observe that $(1.5)$ yields $\left(A^{\prime}, 0\right)-\left(B^{\prime}, 0\right)=$ $2 a \otimes e_{1}$. Thanks to the convexity of $\omega$, we can apply [16, Theorem 3.3] to deduce that $u$ is of the form (2.4). From (2.4), (2.6), and the convexity of $\omega$, it readily follows that $\chi_{F}=\chi_{E \times I} \mathcal{L}^{3}$-a.e. in $\Omega$ for some set $E \subset \omega$ of finite perimeter in $\omega$ satisfying (2.5).

Step 2. We now consider the case $A^{\prime}=B^{\prime}$. Given $(u, b) \in \mathscr{C}$, we have $b \in$ $B V\left(\Omega ;\left\{A_{3}, B_{3}\right\}\right)$, so that $b=\left(1-\chi_{F}\right) A_{3}+\chi_{F} B_{3}$ for some set $F \subset \Omega$ of finite perimeter in $\Omega$. By standard slicing results (see[4, Section 3.11]), $b^{x^{\prime}}:=b\left(x^{\prime}, \cdot\right)$ belongs to $B V\left(I ; \mathbb{R}^{3}\right)$ for $\mathcal{L}^{2}$-a.e. $x^{\prime} \in \omega$, and $\mathcal{L}^{2}\left\lfloor\omega \otimes D b^{x^{\prime}}=\partial_{3} b\right.$. Since $\partial_{3} b=0$, we deduce that $D b^{x^{\prime}}=0$ for $\mathcal{L}^{2}$-a.e. $x^{\prime} \in \omega$. On the other hand, we can find a representative $b^{*}$ of $b$ such that $\left(b^{*}\right)^{x^{\prime}}:=b^{*}\left(x^{\prime}, \cdot\right)$ is a good representative of $b^{x^{\prime}}$ for $\mathcal{L}^{2}$-a.e. $x^{\prime} \in \omega$. Since $D b^{x^{\prime}}=0$, we conclude that $\left(b^{*}\right)^{x^{\prime}}$ is constant for $\mathcal{L}^{2}$-a.e. $x^{\prime} \in \omega$, that is $b^{*}(x)=b^{*}\left(x^{\prime}\right)$. Then it follows that $\chi_{F}=\chi_{E \times I} \mathcal{L}^{3}$-a.e. in $\Omega$ for some set $E \subset \omega$ of finite perimeter in $\omega$.

\section{Compactness}

This section is devoted to the proof of Theorem 1.1, and we assume that $\left(H_{1}\right)$ and $\left(H_{2}\right)$ hold. We consider arbitrary sequences $h_{n} \rightarrow 0^{+}, \varepsilon_{n} \rightarrow 0^{+}$as $n \rightarrow \infty$, and $\left\{u_{n}\right\}_{n \in \mathbb{N}} \subset H^{2}\left(\Omega ; \mathbb{R}^{3}\right)$ such that $\sup _{n} F_{\varepsilon_{n}}^{h_{n}}\left(u_{n}\right)<\infty$. Throughout this section we write $b_{n}:=\frac{1}{h_{n}} \partial_{3} u_{n}$.

Proof of Theorem 1.1. Step 1. We claim that there exist a subsequence $\left\{\varepsilon_{n}\right\}$ (not relabeled), a pair $(u, b) \in W^{1, \infty}\left(\Omega ; \mathbb{R}^{3}\right) \times L^{\infty}\left(\Omega ; \mathbb{R}^{3}\right)$ satisfying $\partial_{3} u=$ 0 , and $\theta \in L^{\infty}(\Omega ;[0,1])$ such that

$u_{n}-f_{\Omega} u_{n} \rightarrow u$ weakly in $W^{1, p}\left(\Omega ; \mathbb{R}^{3}\right), b_{n} \rightarrow b$ weakly in $L^{p}\left(\Omega ; \mathbb{R}^{3}\right)$ as $n \rightarrow \infty$,

and

$$
\left(\nabla^{\prime} u, b\right)(x)=(1-\theta(x)) A+\theta(x) B \quad \text { for } \mathcal{L}^{3} \text {-a.e. } x \in \Omega .
$$

Indeed, we first deduce from the growth assumption $\left(H_{2}\right)$ that

$$
\int_{\Omega}\left(\left|\nabla^{\prime} u_{n}\right|^{p}+\left|b_{n}\right|^{p}\right) d x \leqslant C\left(\int_{\Omega} W\left(\nabla^{\prime} u_{n}, b_{n}\right) d x+1\right) \leqslant C\left(\varepsilon_{n} F_{\varepsilon_{n}}^{h_{n}}\left(u_{n}\right)+1\right) \leqslant C .
$$

Therefore $\left\{b_{n}\right\}$ is uniformly bounded in $L^{p}\left(\Omega ; \mathbb{R}^{3}\right)$, and $\left\{u_{n}-f_{\Omega} u_{n}\right\}$ is uniformly bounded in $W^{1, p}\left(\Omega ; \mathbb{R}^{3}\right)$, thanks to the Poincaré-Wirtinger Inequality. Hence we may extract a subsequence such that (3.1) holds for some pair $(u, b) \in W^{1, p}\left(\Omega ; \mathbb{R}^{3}\right) \times L^{p}\left(\Omega ; \mathbb{R}^{3}\right)$. Since $\left\|\partial_{3} u_{n}\right\|_{L^{p}(\Omega)} \leqslant C h_{n}$, we deduce that $\partial_{3} u \equiv 0$.

Next we observe that the sequence $\left\{\left(\nabla^{\prime} u_{n}, b_{n}\right)\right\}$ also generates a Young measure $\left\{\nu_{x}\right\}_{x \in \Omega}$. From the fundamental theorem on Young measures (see e.g. [33, Theorem 6.11]), we derive 


$$
\int_{\Omega} \int_{\mathbb{R}^{3 \times 3}} W(\xi) d \nu_{x}(\xi) d x \leqslant \lim _{n \rightarrow+\infty} \int_{\Omega} W\left(\nabla^{\prime} u_{n}, b_{n}\right) d x=0,
$$

so that $\operatorname{supp} \nu_{x} \subset\{A, B\}$ for $\mathcal{L}^{3}$-a.e. $x \in \Omega$. Hence there exists $\theta \in L^{1}(\Omega ;[0,1])$ such that

$$
\nu_{x}=(1-\theta(x)) \delta_{\xi=A}+\theta(x) \delta_{\xi=B} \quad \text { for } \mathcal{L}^{3} \text {-a.e. } x \in \Omega .
$$

Multiplying this last equality by $\xi$ and integrating with respect to $\xi$ yields (3.2), which completes the proof of the claim.

Step 2. We claim that $(u, b) \in \mathscr{C}$. We shall distinguish two distinct cases.

Case a). We first assume that $A^{\prime} \neq B^{\prime}$. For $M>0$ and $\xi^{\prime} \in \mathbb{R}^{3 \times 2}$, we define

$$
\begin{gathered}
\varphi\left(\xi^{\prime}\right):=\inf \left\{\int_{0}^{1} \min \left(\sqrt{W_{0}(g(s))}, M\right)\left|g^{\prime}(s)\right| d s: g \in W^{1, \infty}\left([0,1] ; \mathbb{R}^{3 \times 2}\right),\right. \\
\left.g(0)=A^{\prime} \text { and } g(1)=\xi^{\prime}\right\},
\end{gathered}
$$

where $W_{0}\left(\xi^{\prime}\right):=\min \left\{W\left(\xi^{\prime}, z\right): z \in \mathbb{R}^{3}\right\}$ is a continuous function of $\xi^{\prime}$. One may easily check that $\varphi$ is Lipchitz continuous, $\varphi\left(\xi^{\prime}\right)=0$ if and only if $\xi^{\prime}=A^{\prime}$, and that

$$
\left|\nabla \varphi\left(\xi^{\prime}\right)\right| \leqslant \min \left\{\sqrt{W_{0}\left(\xi^{\prime}\right)}, M\right\} \quad \text { for } \mathcal{L}^{3 \times 2} \text {-a.e. } \xi^{\prime} \in \mathbb{R}^{3 \times 2} .
$$

We claim that $\left\{\varphi\left(\nabla^{\prime} u_{n}\right)\right\}$ is uniformly bounded in $W^{1,1}(\Omega ; \mathbb{R})$. Indeed, estimate first

$$
\int_{\Omega}\left|\nabla\left(\varphi\left(\nabla^{\prime} u_{n}\right)\right)\right| d x \leqslant \int_{\Omega} \sqrt{W_{0}\left(\nabla^{\prime} u_{n}(x)\right)}\left|\nabla\left(\nabla^{\prime} u_{n}\right)\right| d x \leqslant \frac{1}{2} F_{\varepsilon_{n}}^{h_{n}}\left(u_{n}\right) \leqslant C,
$$

and by (3.3),

$$
\int_{\Omega}\left|\varphi\left(\nabla^{\prime} u_{n}(x)\right)\right| d x \leqslant M \int_{\Omega}\left|\nabla^{\prime} u_{n}(x)\right| d x+\varphi(0) \mathcal{L}^{3}(\Omega) .
$$

Hence, up to a further subsequence (not relabeled),

$$
\varphi\left(\nabla^{\prime} u_{n}\right) \rightarrow H \quad \text { in } L^{1}(\Omega) \text { as } n \rightarrow \infty,
$$

for some $H \in B V(\Omega)$. On the other hand, the Young measure $\left\{\mu_{x}\right\}_{x \in \Omega}$ generated by $\left\{\varphi\left(\nabla^{\prime} u_{n}\right)\right\}$ is given by

$$
\mu_{x}=(1-\theta(x)) \delta_{t=\varphi\left(A^{\prime}\right)}+\theta(x) \delta_{t=\varphi\left(B^{\prime}\right)} .
$$

Then the strong convergence in (3.4) yields $\mu_{x}=\delta_{t=H(x)}$, so that

$$
\delta_{t=H(x)}=(1-\theta(x)) \delta_{t=\varphi\left(A^{\prime}\right)}+\theta(x) \delta_{t=\varphi\left(B^{\prime}\right)} .
$$

As a consequence $\theta(x) \in\{0,1\}$ for $\mathcal{L}^{3}$-a.e. $x \in \Omega$, and

$$
H(x)=(1-\theta(x)) \varphi\left(A^{\prime}\right)+\theta(x) \varphi\left(B^{\prime}\right)=\theta(x) \varphi\left(B^{\prime}\right) \quad \text { for } \mathcal{L}^{3} \text {-a.e. } x \in \Omega .
$$

Since $\varphi\left(B^{\prime}\right) \neq 0$, it yields $\theta \in B V(\Omega ;\{0,1\})$, and we may now write $\theta=\chi_{F}$ for some set $F \subset \Omega$ of finite perimeter in $\Omega$. In view of $(3.2)$, we obtain $\left(\nabla^{\prime} u, b\right)=$ $\left(1-\chi_{F}\right) A+\chi_{F} B \mathcal{L}^{3}$-a.e. in $\Omega$, and thus $\left(\nabla^{\prime} u, b\right)$ belongs to $B V(\Omega ;\{A, B\})$. Since $\partial_{3} u=0$, we have $\nabla u \in B V\left(\Omega ;\left\{\left(A^{\prime}, 0\right),\left(B^{\prime}, 0\right)\right\}\right)$ and it follows from 
[16, Theorem 3.3] that $F=E \times I$ for some set $E \subset \omega$ of finite perimeter in $\omega$, which in turn implies that $\partial_{3} b=0$, and thus $(u, b) \in \mathscr{C}$.

Case $b$ ). Let us now assume that $A^{\prime}=B^{\prime}$ and $A_{3} \neq B_{3}$. For $M>0$ and $z \in \mathbb{R}^{3}$, we define

$$
\begin{gathered}
\psi(z):=\inf \left\{\int_{0}^{1} \min \left(\sqrt{W_{1}(g(s))}, M\right)\left|g^{\prime}(s)\right| d s: g \in W^{1, \infty}\left([0,1] ; \mathbb{R}^{3}\right),\right. \\
\left.g(0)=A_{3} \text { and } g(1)=z\right\}
\end{gathered}
$$

where $W_{1}(z):=\min \left\{W\left(\xi^{\prime}, z\right): \xi^{\prime} \in \mathbb{R}^{3 \times 2}\right\}$ is a continuous function of $z$. As previously $\psi$ is Lipschitz continuous, and $\psi(z)=0$ if and only if $z=A_{3}$. Arguing as in Case a), we obtain that $\left\{\psi\left(b_{n}\right)\right\}$ is uniformly bounded in $W^{1,1}(\Omega ; \mathbb{R})$, and

$$
\frac{1}{h_{n}} \int_{\Omega}\left|\partial_{3}\left(\psi\left(b_{n}\right)\right)\right| d x \leqslant \frac{1}{h_{n}} \int_{\Omega} \sqrt{W_{1}\left(b_{n}\right)}\left|\partial_{3} b_{n}\right| d x \leqslant \frac{1}{2} F_{\varepsilon_{n}}^{h_{n}}\left(u_{n}\right) \leqslant C .
$$

Therefore, up to a subsequence,

$$
\psi\left(b_{n}\right) \rightarrow G \quad \text { in } L^{1}(\Omega) \text { as } n \rightarrow \infty,
$$

for some $G \in B V(\Omega)$ satisfying $\partial_{3} G=0$. Arguing again as in Case a), we deduce from (3.5) that

$$
\delta_{t=G(x)}=(1-\theta(x)) \delta_{t=\psi\left(A_{3}\right)}+\theta(x) \delta_{t=\psi\left(B_{3}\right)},
$$

which yields $\theta \in B V(\Omega ;\{0,1\})$. Since $\partial_{3} G=0$ we can argue as in the proof of Theorem 2.1, Step 2, to deduce that $\theta(x)=\chi_{E}\left(x^{\prime}\right)$ for some set $E \subset \omega$ of finite perimeter in $\omega$. In view of $(3.2)$, we conclude that $\left(\nabla^{\prime} u, b\right) \in B V(\Omega ;\{A, B\})$ and $\partial_{3} b=0$, and thus $(u, b) \in \mathscr{C}$.

Step 3. In view of the previous steps, we know that $\left(\nabla u_{n}, b_{n}\right) \rightarrow(\nabla u, b)$ weakly in $L^{p}(\Omega)$, and that $\left\{\left(\nabla^{\prime} u_{n}, b_{n}\right)\right\}_{n \in \mathbb{N}}$ generates the Young measure $\left\{\nu_{x}\right\}_{x \in \Omega}$ given by

$$
\nu_{x}(\xi)=\left(1-\chi_{E}\left(x^{\prime}\right)\right) \delta_{\xi=A}+\chi_{E}\left(x^{\prime}\right) \delta_{\xi=B}=\delta_{\xi=\left(\nabla^{\prime} u, b\right)} .
$$

By standard results on Young measures (see e.g. [33, Proposition 6.12]), it follows that $\left(\nabla^{\prime} u_{n}, b_{n}\right) \rightarrow\left(\nabla^{\prime} u, b\right)$ strongly in $L^{p}\left(\Omega ; \mathbb{R}^{3 \times 3}\right)$, and the proof of Theorem 1.1 is complete.

\section{4. $\Gamma$-convergence in the critical regime}

This section is devoted to the proof of Theorem 1.2. The $\Gamma$-liminf and $\Gamma$-limsup inequalities are derived in Theorems 4.6 and 4.11 respectively, while Corollary 4.10 shows that the lower and upper inequalities actually coincide. In proving lower inequalities, we partially adopt the approach of [16], once adapted to the dimension reduction setting. Throughout this section the parameter $\gamma \in(0, \infty)$ is given. 


\subsection{The $\Gamma$ - lim inf inequality}

We introduce the constant

$$
\begin{array}{r}
K_{\gamma}^{\star}:=\inf \left\{\liminf _{n \rightarrow \infty} F_{\varepsilon_{n}}^{h_{n}}\left(u_{n}, Q\right): h_{n} \rightarrow 0^{+} \text {and } \varepsilon_{n} \rightarrow 0^{+} \text {with } h_{n} / \varepsilon_{n} \rightarrow \gamma,\right. \\
\left.\left\{u_{n}\right\} \subset H^{2}\left(Q ; \mathbb{R}^{3}\right),\left(u_{n}, \frac{1}{h_{n}} \partial_{3} u_{n}\right) \rightarrow\left(u_{0}, b_{0}\right) \text { in }\left[L^{1}\left(Q ; \mathbb{R}^{3}\right)\right]^{2}\right\},
\end{array}
$$

where the functions $u_{0}$ and $b_{0}$ are given by (2.1). The constant $K_{\gamma}^{\star}$ turns out to be finite, as one may check by considering an admissible sequence $\left\{u_{n}\right\}$ made of suitable regularizations of $u_{0}$ and $b_{0}$ (see also the proof of Theorem 4.11). In this subsection we shall prove that under assumptions $\left(H_{1}\right)-\left(H_{2}\right)$ and $\left(H_{5}\right)$, the lower $\Gamma$-limit evaluated at any $(u, b) \in \mathscr{C}$ is bounded from below by $K_{\gamma}^{\star}$ times the length of the jump set of $\left(\nabla^{\prime} u, b\right)$. We first prove this statement in the case of an elementary jump set.

Proposition 4.1. Assume that $\left(H_{1}\right),\left(H_{2}\right)$ and $(1.5)$ hold. Let $h_{n} \rightarrow 0^{+}$and $\varepsilon_{n} \rightarrow 0^{+}$be arbitrary sequences such that $h_{n} / \varepsilon_{n} \rightarrow \gamma$. Let $\rho>0$ and $\alpha \in \mathbb{R}$, let $J \subset \mathbb{R}$ be a bounded open interval, and consider the cylinder $U:=(\alpha-\rho, \alpha+\rho)$ $\times J \times I$. Let $(u, b) \in W^{1, \infty}\left(U ; \mathbb{R}^{3}\right) \times L^{\infty}\left(U ; \mathbb{R}^{3}\right)$ satisfying $\partial_{3} u=\partial_{3} b=0$, and

$$
\left(\nabla^{\prime} u, b\right)=\chi_{\left\{x_{1}<\alpha\right\}} B+\chi_{\left\{x_{1} \geqslant \alpha\right\}} A \quad \text { or } \quad\left(\nabla^{\prime} u, b\right)=\chi_{\left\{x_{1} \leqslant \alpha\right\}} A+\chi_{\left\{x_{1}>\alpha\right\}} B .
$$

Then for any sequence $\left\{u_{n}\right\} \subset H^{2}\left(U ; \mathbb{R}^{3}\right)$ satisfying $\left(u_{n}, \frac{1}{h_{n}} \partial_{3} u_{n}\right) \rightarrow(u, b)$ in $\left[L^{1}\left(U ; \mathbb{R}^{3}\right)\right]^{2}$, we have

$$
\liminf _{n \rightarrow \infty} F_{\varepsilon_{n}}^{h_{n}}\left(u_{n}, U\right) \geqslant K_{\gamma}^{\star} \mathcal{H}^{1}(J) .
$$

The proof of Proposition 4.1 relies on scaling properties and the translation invariance of the energy functional $F_{\varepsilon}^{h}$. To determine the corresponding properties in the limit we introduce the following set function. For an open bounded set $J \subset \mathbb{R}$ and $\rho>0$, we write $J_{\rho}:=\rho I \times J \times I$, and we define

$$
\begin{array}{r}
\mathcal{E}_{\gamma}(J, \rho):=\inf \left\{\liminf _{n \rightarrow \infty} F_{\varepsilon_{n}}^{h_{n}}\left(u_{n}, J_{\rho}\right): h_{n} \rightarrow 0^{+} \text {and } \varepsilon_{n} \rightarrow 0^{+} \text {with } h_{n} / \varepsilon_{n} \rightarrow \gamma,\right. \\
\left.\left\{u_{n}\right\} \subset H^{2}\left(J_{\rho} ; \mathbb{R}^{3}\right),\left(u_{n}, \frac{1}{h_{n}} \partial_{3} u_{n}\right) \rightarrow\left(u_{0}, b_{0}\right) \text { in }\left[L^{1}\left(J_{\rho} ; \mathbb{R}^{3}\right)\right]^{2}\right\} .
\end{array}
$$

Noticing that $K_{\gamma}^{\star}=\mathcal{E}_{\gamma}(I, 1)$ we now state the following lemma.

Lemma 4.2. Assume that $\left(H_{1}\right),\left(H_{2}\right)$ and (1.5) hold. Then

(i) $\mathcal{E}_{\gamma}(t+J, \rho)=\mathcal{E}_{\gamma}(J, \rho)$ for all $t \in \mathbb{R}$;

(ii) if $J_{1} \subset J_{2}$ and $\rho_{1} \leqslant \rho_{2}$, then $\mathcal{E}_{\gamma}\left(J_{1}, \rho_{1}\right) \leqslant \mathcal{E}_{\gamma}\left(J_{2}, \rho_{2}\right)$;

(iii) if $J_{1} \cap J_{2}=\emptyset$, then $\mathcal{E}_{\gamma}\left(J_{1} \cup J_{2}, \rho\right) \geqslant \mathcal{E}_{\gamma}\left(J_{1}, \rho\right)+\mathcal{E}_{\gamma}\left(J_{2}, \rho\right)$;

(iv) if $\alpha>0$, then $\mathcal{E}_{\gamma}(\alpha J, \alpha \rho)=\alpha \mathcal{E}_{\gamma}(J, \rho)$;

(v) if $0<\alpha<1$, then $\mathcal{E}_{\gamma}(\alpha J, \rho) \geqslant \alpha \mathcal{E}_{\gamma}(J, \rho)$;

(vi) if $J$ is an interval then $\mathcal{E}_{\gamma}(J, \rho)=\mathcal{H}^{1}(J) \mathcal{E}_{\gamma}(I, \rho)$;

(vii) if $J$ is an interval then $\mathcal{E}_{\gamma}(J, \rho)=\mathcal{E}_{\gamma}(J, \delta)$ for all $\delta>0$. 
Proof. We first observe that (i) follows from the translation invariance of the functional $F_{\varepsilon}^{h}$. Then (ii) is due to the fact that any admissible sequence for $\mathcal{E}_{\gamma}\left(J_{2}, \rho_{2}\right)$ yields an admissible sequence for $\mathcal{E}_{\gamma}\left(J_{1}, \rho_{1}\right)$ whenever $J_{1} \subset J_{2}$ and $\rho_{1} \leqslant \rho_{2}$. The proof of claim (iii) follows a similar argument.

Proof of (iv). Let $h_{n} \rightarrow 0^{+}$and $\varepsilon_{n} \rightarrow 0^{+}$be such that $h_{n} / \varepsilon_{n} \rightarrow \gamma$, and let $\left\{u_{n}\right\}$ be an admissible sequence for $\mathcal{E}_{\gamma}(\alpha J, \alpha \rho)$. We define for $x \in J_{\rho}, v_{n}(x):=$ $\frac{1}{\alpha} u_{n}\left(\alpha x^{\prime}, x_{3}\right), \tilde{h}_{n}:=h_{n} / \alpha$ and $\tilde{\varepsilon}_{n}:=\varepsilon_{n} / \alpha$. By homogeneity of $u_{0}$ and $b_{0}$, we infer that $v_{n}(x) \rightarrow \frac{1}{\alpha} u_{0}\left(\alpha x^{\prime}, x_{3}\right)=u_{0}(x)$ and $\frac{1}{\tilde{h}_{n}} \partial_{3} v_{n} \rightarrow b_{0}$ in $L^{1}\left(J_{\rho} ; \mathbb{R}^{3}\right)$ as $n \rightarrow \infty$. In particular, $\left\{v_{n}\right\}$ with $\left\{\left(\tilde{h}_{n}, \tilde{\varepsilon}_{n}\right)\right\}$ is admissible for $\mathcal{E}_{\gamma}(J, \rho)$. Changing variables then yields $F_{\tilde{\varepsilon}_{n}}^{\tilde{h}_{n}}\left(v_{n}, J_{\rho}\right)=\frac{1}{\alpha} F_{\varepsilon_{n}}^{h_{n}}\left(u_{n},(\alpha J)_{\alpha \rho}\right)$. Letting $n \rightarrow \infty$ we deduce that $\liminf \inf _{n} F_{\varepsilon_{n}}^{h_{n}}\left(u_{n},(\alpha J)_{\alpha \rho}\right) \geqslant \alpha \mathcal{E}_{\gamma}(J, \rho)$. In view of the arbitrariness of $\left\{\left(h_{n}, \varepsilon_{n}\right)\right\}$ and $\left\{u_{n}\right\}$, we conclude that $\mathcal{E}_{\gamma}(\alpha J, \alpha \rho) \geqslant \alpha \mathcal{E}_{\gamma}(J, \rho)$. The reverse inequality follows from the arbitrariness of $\alpha>0$.

Proof of (v). If $0<\alpha<1$ then $(\alpha J)_{\alpha \rho} \subset(\alpha J)_{\rho}$, and we derive from (ii) and (iv) that $\mathcal{E}_{\gamma}(\alpha J, \rho) \geqslant \mathcal{E}_{\gamma}(\alpha J, \alpha \rho)=\alpha \mathcal{E}_{\gamma}(J, \rho)$.

Proof of (vi). Write $J=Z \cup\left(\bigcup_{k=1}^{N} J_{k}\right)$ for some finite set $Z$ and some family $\left\{J_{k}\right\}_{k=1}^{N}$ of mutually disjoint open intervals of the form $J_{k}=a_{k}+\alpha_{k} I$ with $0<\alpha_{k}<1$. In particular, $\mathcal{H}^{1}(J)=\sum_{k=1}^{N} \alpha_{k}$. We infer from (i), (iii) and (v) that $\mathcal{E}_{\gamma}(J, \rho) \geqslant\left(\sum_{k} \alpha_{k}\right) \mathcal{E}_{\gamma}(I, \rho)$, leading to $\mathcal{E}_{\gamma}(J, \rho) \geqslant \mathcal{H}^{1}(J) \mathcal{E}_{\gamma}(I, \rho)$. The reverse inequality can be obtained in the same way inverting the roles of $I$ and $J$.

Proof of (vii). Claim (vii) is a straightforward consequence of (iv) and (vi).

Remark 4.3. As a consequence of (vi) and (vii) in Lemma 4.2, we have $\mathcal{E}_{\gamma}(J, \rho)=K_{\gamma}^{\star} \mathcal{H}^{1}(J)$ for every $\rho>0$ and every bounded open interval $J \subset \mathbb{R}$.

An important consequence of Lemma 4.2 is that the energy of optimal sequences for $\mathcal{E}_{\gamma}(J, \rho)$ is concentrated near the limiting interface. We shall make use of Corollary 4.4 in the next subsection in order to compare the constants $K_{\gamma}^{\star}$ and $K_{\gamma}$.

Corollary 4.4. Assume that $\left(H_{1}\right),\left(H_{2}\right)$ and (1.5) hold. Let $0<\delta<\rho$ and let $J \subset \mathbb{R}$ be a bounded open interval. For any sequences $h_{n} \rightarrow 0^{+}, \varepsilon_{n} \rightarrow 0^{+}$ and $\left\{u_{n}\right\} \subset H^{2}\left(J_{\rho} ; \mathbb{R}^{3}\right)$ such that $h_{n} / \varepsilon_{n} \rightarrow \gamma,\left(u_{n}, \frac{1}{h_{n}} \partial_{3} u_{n}\right) \rightarrow\left(u_{0}, b_{0}\right)$ in $\left[L^{1}\left(J_{\rho} ; \mathbb{R}^{3}\right)\right]^{2}$, and $\lim _{n} F_{\varepsilon_{n}}^{h_{n}}\left(u_{n}, J_{\rho}\right)=K_{\gamma}^{\star} \mathcal{H}^{1}(J)$, we have

$$
\lim _{n \rightarrow \infty} F_{\varepsilon_{n}}^{h_{n}}\left(u_{n}, J_{\rho} \backslash J_{\delta}\right)=0 .
$$

Proof. Since $J_{\delta} \subset J_{\rho}$, we deduce from Remark 4.3 that $\lim \sup _{n} F_{\varepsilon_{n}}^{h_{n}}\left(u_{n}, J_{\delta}\right) \leqslant$ $\mathcal{E}_{\gamma}(J, \rho)<\infty$. On the other hand the sequence $\left\{u_{n}\right\}$ is admissible for $\mathcal{E}_{\gamma}(J, \delta)$. In view of Lemma 4.2 , we infer that

$$
\liminf _{n \rightarrow \infty} F_{\varepsilon_{n}}^{h_{n}}\left(u_{n}, J_{\delta}\right) \geqslant \mathcal{E}_{\gamma}(J, \delta)=\mathcal{E}_{\gamma}(J, \rho)=\lim _{n \rightarrow \infty} F_{\varepsilon_{n}}^{h_{n}}\left(u_{n}, J_{\rho}\right) .
$$

Therefore $\lim _{n} F_{\varepsilon_{n}}^{h_{n}}\left(u_{n}, J_{\delta}\right)=\lim _{n} F_{\varepsilon_{n}}^{h_{n}}\left(u_{n}, J_{\rho}\right)$ which clearly implies the announced result. 
Proof of Proposition 4.1. By the translation invariance of $F_{\varepsilon_{n}}^{h_{n}}$, we have $\liminf { }_{n} F_{\varepsilon_{n}}^{h_{n}}\left(u_{n}, U\right)=\liminf _{n} F_{\varepsilon_{n}}^{h_{n}}\left(\tau u_{n}, J_{\rho}\right)$, where $\tau u_{n}(x):=u_{n}\left(x_{1}+\right.$ $\left.\alpha, x_{2}, x_{3}\right)$. Obviously $\left(\tau u_{n}, \frac{1}{h_{n}} \partial_{3} \tau u_{n}\right) \rightarrow(\tau u, \tau b)$ in $\left[L^{1}\left(J_{\rho} ; \mathbb{R}^{3}\right)\right]^{2}$ with $\tau u(x):=$ $u\left(x_{1}+\alpha, x_{2}, x_{3}\right)$ and $\tau b(x):=b\left(x_{1}+\alpha, x_{2}, x_{3}\right)$. If the first case in (4.2) holds, then $(\tau u, \tau b)=\left(u_{0}+c_{0}, b_{0}\right)$ for some constant $c_{0} \in \mathbb{R}^{3}$. Subtracting the constant $c_{0}$, we derive from the definition of $\mathcal{E}_{\gamma}$ and Remark 4.3 that

$$
\liminf _{n \rightarrow \infty} F_{\varepsilon_{n}}^{h_{n}}\left(u_{n}, U\right)=\liminf _{n \rightarrow \infty} F_{\varepsilon_{n}}^{h_{n}}\left(\tau u_{n}-c_{0}, J_{\rho}\right) \geqslant \mathcal{E}_{\gamma}(J, \rho)=K_{\gamma}^{\star} \mathcal{H}^{1}(J) .
$$

If the alternative case in (4.2) holds, then $(-\tau u, \tau b)(-x)=\left(u_{0}+c_{0}, b_{0}\right)(x)$ for some constant $c_{0} \in \mathbb{R}^{3}$. Observe that $F_{\varepsilon_{n}}^{h_{n}}\left(\tau u_{n}, J_{\rho}\right)=F_{\varepsilon_{n}}^{h_{n}}\left(v_{n}, J_{\rho}\right)$ with $v_{n}(x)=-\tau u_{n}(-x)-c_{0}$. Then $\left(v_{n}, \frac{1}{h_{n}} \partial_{3} v_{n}\right) \rightarrow\left(u_{0}, b_{0}\right)$ in $\left[L^{1}\left(J_{\rho} ; \mathbb{R}^{3}\right)\right]^{2}$. Hence,

$$
\liminf _{n \rightarrow \infty} F_{\varepsilon_{n}}^{h_{n}}\left(u_{n}, J_{\rho}\right)=\liminf _{n \rightarrow \infty} F_{\varepsilon_{n}}^{h_{n}}\left(v_{n}, J_{\rho}\right) \geqslant \mathcal{E}_{\gamma}(J, \rho)=K_{\gamma}^{\star} \mathcal{H}^{1}(J),
$$

and the proof is complete.

Remark 4.5. Setting $K_{\infty}^{\star}$ to be the constant defined by (4.1) with $\gamma=+\infty$ (see (6.1)), and assuming that $K_{\infty}^{\star}<\infty$, one may check that Proposition 4.1 and Corollary 4.4 still hold in the case $\gamma=+\infty$ with the same proofs.

We are now ready to prove the main result of this subsection which extends Proposition 4.1 to the general case. The proof for $A^{\prime} \neq B^{\prime}$ will be a direct consequence of Proposition 4.1, while the case $A^{\prime}=B^{\prime}$ will require an additional analysis based on a blow-up argument.

Theorem 4.6. Assume that $\left(H_{1}\right)-\left(H_{2}\right),\left(H_{5}\right)$ and $(1.5)$ hold. Let $h_{n} \rightarrow 0^{+}$and $\varepsilon_{n} \rightarrow 0^{+}$be arbitrary sequences such that $h_{n} / \varepsilon_{n} \rightarrow \gamma$. Then, for any $(u, b) \in$ $\mathscr{C}$ and any sequences $\left\{u_{n}\right\} \subset H^{2}\left(\Omega ; \mathbb{R}^{3}\right)$ such that $\left(u_{n}, \frac{1}{h_{n}} \partial_{3} u_{n}\right) \rightarrow(u, b)$ in $\left[L^{1}\left(\Omega ; \mathbb{R}^{3}\right)\right]^{2}$, we have

$$
\liminf _{n \rightarrow \infty} F_{\varepsilon_{n}}^{h_{n}}\left(u_{n}\right) \geqslant K_{\gamma}^{\star} \operatorname{Per}_{\omega}(E)
$$

where $\left(\nabla^{\prime} u, b\right)(x)=\left(1-\chi_{E}\left(x^{\prime}\right)\right) A+\chi_{E}\left(x^{\prime}\right) B$.

Proof. Step 1. We first assume that $A^{\prime} \neq B^{\prime}$. Assuming that $E$ is non trivial, by Theorem 2.1 we can write $\partial^{*} E \cap \omega$ as in $(2.5)$. Then we have $\mathcal{H}^{1}\left(\partial^{*} E \cap\right.$ $\omega)=\sum_{i \in \mathscr{I}} \mathcal{H}^{1}\left(J_{i}\right)<\infty$. Consider an arbitrarily small $\delta>0$ and choose $k^{-}=k^{-}(\delta) \in \mathscr{I}$ and $k^{+}=k^{+}(\delta) \in \mathscr{I}$ such that $k^{-} \leqslant k^{+}$, and $\mathcal{H}^{1}\left(\partial^{*} E \cap \omega\right) \leqslant$ $\sum_{i=k^{-}}^{k^{+}} \mathcal{H}^{1}\left(J_{i}\right)+\delta$. For each $i=k^{-}, \ldots, k^{+}$, let $J_{i}^{\prime} \subset \subset J_{i}$ be an open interval satisfying $\mathcal{H}^{1}\left(J_{i}\right) \leqslant \mathcal{H}^{1}\left(J_{i}^{\prime}\right)+\frac{\delta}{k^{+}-k^{-}+1}$. Since $\left\{\alpha_{i}\right\} \times J_{i}^{\prime} \subset \subset \omega$ and $\alpha_{i}<\alpha_{i+1}$, we may find $\rho>0$ small in such a way that the sets $\left(\alpha_{i}-\rho, \alpha_{i}+\rho\right) \times J_{i}^{\prime}$ are still compactly contained in $\omega$, and $\alpha_{i}+\rho<\alpha_{i+1}-\rho$ for $i=k^{-}, \ldots, k^{+}$. Then we set for each $i=k^{-}, \ldots, k^{+}, U_{i}:=\left(\alpha_{i}-\rho, \alpha_{i}+\rho\right) \times J_{i}^{\prime} \times I \subset \Omega$. Observe that the $U_{i}$ 's are pairwise disjoint, and that $\left(\nabla^{\prime} u, b\right)$ is of the form (4.2) in each $U_{i}$.

Let us now fix an arbitrary sequence $\left\{u_{n}\right\} \subset H^{2}\left(\Omega ; \mathbb{R}^{3}\right)$ such that $\left(u_{n}, \frac{1}{h_{n}} \partial_{3} u_{n}\right) \rightarrow(u, b)$ in $\left[L^{1}\left(\Omega ; \mathbb{R}^{3}\right)\right]^{2}$. Using Proposition 4.1 , we estimate 


$$
\begin{aligned}
\liminf _{n \rightarrow \infty} F_{\varepsilon_{n}}^{h_{n}}\left(u_{n}\right) & \geqslant \sum_{i=k^{-}}^{k^{+}} \liminf _{n \rightarrow \infty} F_{\varepsilon_{n}}^{h_{n}}\left(u_{n}, U_{i}\right) \\
& \geqslant \sum_{i=k^{-}}^{k^{+}} K_{\gamma}^{\star} \mathcal{H}^{1}\left(J_{i}^{\prime}\right) \geqslant K_{\gamma}^{\star} \mathcal{H}^{1}\left(\partial^{*} E \cap \omega\right)-2 \delta K_{\gamma}^{\star},
\end{aligned}
$$

and the conclusion follows letting $\delta \rightarrow 0$.

Step 2. We now consider the case $A^{\prime}=B^{\prime}\left(=0\right.$ by (1.5)). Then $u_{0}=0$ and $u$ is constant. Without loss of generality we may assume that $u \equiv 0$. In the remaining of this proof, we shall identify any $b \in L^{1}\left(Q^{\prime} ; \mathbb{R}^{3}\right)$ with its extension to $Q$ given by $b(x)=b\left(x^{\prime}\right)$. With this convention, we introduce for $b \in L^{1}\left(Q^{\prime} ; \mathbb{R}^{3}\right)$,

$$
\begin{array}{r}
\mathcal{G}_{\gamma}(b):=\inf \left\{\liminf _{n \rightarrow \infty} F_{\varepsilon_{n}}^{h_{n}}\left(u_{n}, Q\right): h_{n} \rightarrow 0 \text { and } \varepsilon_{n} \rightarrow 0 \text { with } h_{n} / \varepsilon_{n} \rightarrow \gamma,\right. \\
\left.\left\{u_{n}\right\} \subset H^{2}\left(Q ; \mathbb{R}^{3}\right),\left(u_{n}, \frac{1}{h_{n}} \partial_{3} u_{n}\right) \rightarrow(0, b) \operatorname{in}\left[L^{1}\left(Q ; \mathbb{R}^{3}\right)\right]^{2}\right\} .
\end{array}
$$

Notice that $K_{\gamma}^{\star}=\mathcal{G}_{\gamma}\left(b_{0}\right)$. We shall require the sequential $L^{1}$-lower semicontinuity of the functional $\mathcal{G}_{\gamma}$ stated below. The proof of Lemma 4.7 only involves a standard diagonalization argument, and we shall omit it.

Lemma 4.7. $\mathcal{G}_{\gamma}$ is sequentially lower semicontinuous for the strong $L^{1}$-topology.

Let $\left\{u_{n}\right\} \subset H^{2}\left(\Omega ; \mathbb{R}^{3}\right)$ be an arbitrary sequence satisfying $\left(u_{n}, \frac{1}{h_{n}} \partial_{3} u_{n}\right) \rightarrow$ $(0, b)$ in $\left[L^{1}\left(\Omega ; \mathbb{R}^{3}\right)\right]^{2}$. Without loss of generality, we may assume that $\liminf _{n} F_{\varepsilon_{n}}^{h_{n}}\left(u_{n}\right)=\lim _{n} F_{\varepsilon_{n}}^{h_{n}}\left(u_{n}\right)<\infty$. By Theorem 2.1 we have $b(x)=$ $b\left(x^{\prime}\right)=\left(1-\chi_{E}\left(x^{\prime}\right)\right) A_{3}+\chi_{E}\left(x^{\prime}\right) B_{3}$ for a set $E \subset \omega$ of finite perimeter in $\omega$.

Using Fubini's theorem, we define a finite nonnegative Radon measure $\mu_{n}$ on $\omega$ by setting

$$
\mu_{n}:=\left(\int_{-\frac{1}{2}}^{\frac{1}{2}} \frac{1}{\varepsilon_{n}} W\left(\nabla_{h_{n}} u_{n}\right)+\varepsilon_{n}\left|\nabla_{h_{n}}^{2} u_{n}\right|^{2} d x_{3}\right) \mathcal{L}^{2}\lfloor\omega,
$$

and $\mu_{n}(\omega)=F_{\varepsilon_{n}}^{h_{n}}\left(u_{n}\right)$. In particular $\sup _{n} \mu_{n}(\omega)<\infty$, and thus there is a subsequence (not relabeled) such that $\mu_{n} \rightarrow \mu$ weakly* in the sense of measures for some finite nonnegative Radon measure $\mu$ on $\omega$. By lower semicontinuity we have $\mu(\omega) \leqslant \lim _{n} \mu_{n}(\omega)=\lim _{n} F_{\varepsilon_{n}}^{h_{n}}\left(u_{n}\right)$. It then suffices to prove that $\mu(\omega) \geqslant K_{\gamma}^{\star} \mathcal{H}^{1}\left(\partial^{*} E \cap \omega\right)$. By the Radon-Nikodým Theorem, we can decompose $\mu$ as $\mu=\mu_{0}+\mu_{s}$, where $\mu_{0}$ and $\mu_{s}$ are mutually singular nonnegative Radon measures on $\omega$, and $\mu_{0} \ll \mathcal{H}^{1}\left\lfloor\partial^{*} E \cap \omega\right.$. It is enough to show that $\mu_{0}(\omega) \geqslant K_{\gamma}^{\star} \mathcal{H}^{1}\left(\partial^{*} E \cap \omega\right)$ which can be obtained by proving that

$$
\frac{d \mu_{0}}{d \mathcal{H}^{1}\left\lfloor\partial^{*} E \cap \omega\right.}\left(x_{0}^{\prime}\right) \geqslant K_{\gamma}^{\star} \quad \text { for } \mathcal{H}^{1} \text {-a.e. } x_{0}^{\prime} \in \partial^{*} E \cap \omega \text {. }
$$

For $\nu \in \mathbb{S}^{1}$ and $\delta>0$, we denote by $Q_{\nu}^{\prime} \subset \mathbb{R}^{2}$ the unit cube centered at the origin with two sides orthogonal to $\nu$, and $Q_{\nu}^{\prime}\left(x_{0}^{\prime}, \delta\right):=x_{0}^{\prime}+\delta Q_{\nu}^{\prime}$. By a generalization of the Besicovitch Differentiation Theorem (see [2, Proposition 
2.2]), there exists a Borel set $Z \subset \omega$ such that $\mathcal{H}^{1}(Z)=0$, the Radon-Nikodým derivative of $\mu_{0}$ with respect to $\mathcal{H}^{1} \partial^{*} E \cap \omega$ exists and is finite at every $x_{0}^{\prime} \in$ $\left(\partial^{*} E \cap \omega\right) \backslash Z$, and

$$
\begin{aligned}
& \frac{d \mu_{0}}{d \mathcal{H}^{1} \partial^{*} E \cap \omega}\left(x_{0}^{\prime}\right)=\lim _{\delta \rightarrow 0^{+}} \frac{\mu_{0}\left(Q_{\nu}^{\prime}\left(x_{0}^{\prime}, \delta\right)\right)}{\mathcal{H}^{1}\left(\partial^{*}\left\lfloor E \cap Q_{\nu}^{\prime}\left(x_{0}^{\prime}, \delta\right)\right)\right.} \quad \text { for every } x_{0}^{\prime} \in\left(\partial^{*} E \cap \omega\right) \backslash Z \\
& \quad \text { and all } \nu \in \mathbb{S}^{1} \text {. }
\end{aligned}
$$

For $x_{0}^{\prime} \in \partial^{*} E \cap \omega$, let us denote by $\nu_{0} \in \mathbb{S}^{1}$ the generalized outer normal to $E$ at $x_{0}^{\prime}$. By Theorem 3.59 and Remark 2.82 in [4], we have

$$
\lim _{\delta \rightarrow 0^{+}} \frac{\mathcal{H}^{1}\left(\partial^{*} E \cap Q_{\nu_{0}}^{\prime}\left(x_{0}^{\prime}, \delta\right)\right)}{\delta}=1 \quad \text { for } \mathcal{H}^{1} \text {-a.e. } x_{0}^{\prime} \in \partial^{*} E \cap \omega .
$$

Moreover, it is well known (see e.g. [4, Example 3.68]) that

$$
\lim _{\delta \rightarrow 0^{+}} \frac{1}{\delta^{2}} \int_{Q_{\nu_{0}}^{\prime}\left(x_{0}^{\prime}, \delta\right)}\left|b\left(x^{\prime}\right)-\bar{b}_{x_{0}}\left(x^{\prime}\right)\right| d x^{\prime}=0 \quad \text { for } \mathcal{H}^{1} \text {-a.e. } x_{0}^{\prime} \in \partial^{*} E \cap \omega
$$

where $\bar{b}_{x_{0}}\left(x^{\prime}\right):=\chi_{\left\{\left(x^{\prime}-x_{0}^{\prime}\right) \cdot \nu_{0}>0\right\}}\left(x^{\prime}\right) A_{3}+\chi_{\left\{\left(x^{\prime}-x_{0}^{\prime}\right) \cdot \nu_{0}<0\right\}}\left(x^{\prime}\right) B_{3}$.

Let us now fix a point $x_{0}^{\prime} \in\left(\partial^{*} E \cap \omega\right) \backslash Z$ satisfying (4.3)-(4.4). We choose a sequence $\delta_{k} \rightarrow 0^{+}$such that $Q_{\nu_{0}}^{\prime}\left(x_{0}^{\prime}, \delta_{k}\right) \subset \omega$ and $\mu\left(\partial Q_{\nu_{0}}^{\prime}\left(x_{0}^{\prime}, \delta_{k}\right)\right)=0$ for all $k \in \mathbb{N}$. Then

$$
\begin{aligned}
\frac{d \mu_{0}}{d \mathcal{H}^{1}\left\lfloor\partial^{*} E \cap \omega\right.}\left(x_{0}^{\prime}\right) & =\lim _{k \rightarrow \infty} \frac{1}{\delta_{k}} \mu\left(Q_{\nu_{0}}^{\prime}\left(x_{0}^{\prime}, \delta_{k}\right)\right)=\lim _{k \rightarrow \infty} \lim _{n \rightarrow \infty} \frac{1}{\delta_{k}} \mu_{n}\left(Q_{\nu_{0}}^{\prime}\left(x_{0}^{\prime}, \delta_{k}\right)\right) \\
& =\lim _{k \rightarrow \infty} \lim _{n \rightarrow \infty} \frac{1}{\delta_{k}} F_{\varepsilon_{n}}^{h_{n}}\left(u_{n}, Q_{\nu_{0}}^{\prime}\left(x_{0}^{\prime}, \delta_{k}\right) \times I\right),
\end{aligned}
$$

where we have used Fubini's theorem in the last equality. Let $R \in S O(2)$ be such that $R e_{1}^{\prime}=\nu_{0}$, and define for $x \in Q, v_{n, k}(x):=\frac{1}{\delta_{k}} u_{n}\left(x_{0}^{\prime}+\delta_{k} R x^{\prime}, x_{3}\right)$. Observe that $\bar{b}_{x_{0}}\left(x_{0}^{\prime}+R x^{\prime}\right)=b_{0}\left(x^{\prime}\right)$. We also have $h_{n, k}:=\frac{h_{n}}{\delta_{k}} \rightarrow 0, \varepsilon_{n, k}:=$ $\frac{\varepsilon_{n}}{\delta_{k}} \rightarrow 0, h_{n, k} / \varepsilon_{n, k} \rightarrow \gamma$, and $\left(v_{n, k}, \frac{1}{h_{n, k}} \partial_{3} v_{n, k}\right) \rightarrow\left(0, b_{k}\right)$ in $\left[L^{1}\left(Q ; \mathbb{R}^{3}\right)\right]^{2}$ where $b_{k}\left(x^{\prime}\right):=b\left(x_{0}^{\prime}+\delta_{k} R^{\prime} x^{\prime}\right)$. Changing variables, we derive from assumption $\left(H_{5}\right)$ that

$$
F_{\varepsilon_{n}}^{h_{n}}\left(u_{n}, Q_{\nu_{0}}^{\prime}\left(x_{0}^{\prime}, \delta_{k}\right) \times I\right)=\delta_{k} F_{\varepsilon_{n, k}}^{h_{n, k}}\left(v_{n, k}, Q\right) .
$$

Then it follows from the definition of $\mathcal{G}_{\gamma}$ that

$$
\frac{d \mu_{0}}{d \mathcal{H}^{1}\left\lfloor\partial^{*} E \cap \omega\right.}\left(x_{0}^{\prime}\right)=\lim _{k \rightarrow \infty} \lim _{n \rightarrow \infty} F_{\varepsilon_{n, k}}^{h_{n, k}}\left(v_{n, k}, Q\right) \geqslant \liminf _{k \rightarrow \infty} \mathcal{G}_{\gamma}\left(b_{k}\right) .
$$

On the other hand, by (4.4) we have $b_{k} \rightarrow b_{0}$ in $L^{1}\left(Q^{\prime} ; \mathbb{R}^{3}\right)$. In view of Lemma 4.7, we deduce that

$$
\frac{d \mu_{0}}{d \mathcal{H}^{1}\left\lfloor\partial^{*} E \cap \omega\right.}\left(x_{0}^{\prime}\right) \geqslant \liminf _{k \rightarrow \infty} \mathcal{G}_{\gamma}\left(b_{k}\right) \geqslant \mathcal{G}_{\gamma}\left(b_{0}\right)=K_{\gamma}^{\star},
$$

which completes the proof. 


\subsection{Lower bound on $K_{\gamma}^{\star}$}

In order to compare the constant $K_{\gamma}^{\star}$ with $K_{\gamma}$, we prove in this subsection that under the additional assumptions $\left(H_{3}\right)-\left(H_{4}\right)$, sequences realizing $K_{\gamma}^{\star}$ can be prescribed near the two sides $\left\{x_{1}= \pm \frac{1}{2}\right\}$, and chosen to be independent of the $x_{2}$-variable. This is the object of Proposition 4.8 below. First we state some useful facts on potentials $W$ satisfying assumptions $\left(H_{1}\right)-\left(H_{3}\right)$ that we shall use throughout the paper. The proof of Lemma 4.8 is elementary and we omit it.

Lemma 4.8. Assume that $\left(H_{1}\right)$ holds. Then $W$ satisfies $\left(H_{2}\right)-\left(H_{3}\right)$ if and only if there exists a constant $C_{*} \geqslant 1$ such that for every $\xi \in \mathbb{R}^{3 \times 3}$,

$$
\frac{1}{C_{*}} \min \left(|\xi-A|^{p},|\xi-B|^{p}\right) \leqslant W(\xi) \leqslant C_{*} \min \left(|\xi-A|^{p},|\xi-B|^{p}\right) .
$$

In particular, if $\left(H_{1}\right)-\left(H_{3}\right)$ hold, then

$$
W(\xi) \leqslant C_{*}^{2} 2^{p-1}\left(W(\bar{\xi})+|\xi-\bar{\xi}|^{p}\right) \quad \forall \xi, \bar{\xi} \in \mathbb{R}^{3 \times 3} .
$$

We now state the pinning condition described above. It parallels [16, Proposition 6.2] in the context of dimension reduction.

Proposition 4.9. Assume that $\left(H_{1}\right)-\left(H_{4}\right)$ and (1.5) hold. Then there exist sequences $\varepsilon_{n} \rightarrow 0,\left\{c_{n}\right\} \subset \mathbb{R}^{3},\left\{g_{n}\right\} \subset C^{2}\left(Q ; \mathbb{R}^{3}\right)$ such that $g_{n}$ is independent of $x_{2}\left(i . e ., g_{n}(x)=: \hat{g}_{n}\left(x_{1}, x_{3}\right)\right), c_{n} \rightarrow 0, g_{n} \rightarrow u_{0}$ in $W^{1, p}\left(Q ; \mathbb{R}^{3}\right), \frac{1}{\gamma \varepsilon_{n}} \partial_{3} g_{n} \rightarrow b_{0}$ in $L^{p}\left(Q ; \mathbb{R}^{3}\right)$,

$$
\begin{aligned}
& g_{n}=u_{0}+\gamma \varepsilon_{n} x_{3} b_{0} \text { in } Q \cap\left\{x_{1}>1 / 4\right\}, \\
& \quad g_{n}=u_{0}+\gamma \varepsilon_{n} x_{3} b_{0}+c_{n} \text { in } Q \cap\left\{x_{1}<-1 / 4\right\},
\end{aligned}
$$

and $\lim _{n} F_{\varepsilon_{n}}^{\gamma \varepsilon_{n}}\left(g_{n}, Q\right)=K_{\gamma}^{\star}$.

Proof. Step 1. Let us consider sequences $h_{n} \rightarrow 0, \tilde{\varepsilon}_{n} \rightarrow 0$ and $\left\{\tilde{u}_{n}\right\} \subset$ $H^{2}\left(Q ; \mathbb{R}^{3}\right)$ such that $h_{n} / \tilde{\varepsilon}_{n} \rightarrow \gamma,\left(\tilde{u}_{n}, \frac{1}{h_{n}} \partial_{3} \tilde{u}_{n}\right) \rightarrow\left(u_{0}, b_{0}\right)$ in $\left[L^{1}\left(Q ; \mathbb{R}^{3}\right)\right]^{2}$, and $\lim _{n} F_{\tilde{\varepsilon}_{n}}^{h_{n}}\left(\tilde{u}_{n}, Q\right)=K_{\gamma}^{\star}$. Applying standard regularization techniques if necessary, we may assume that $\tilde{u}_{n} \in C^{2}\left(Q ; \mathbb{R}^{3}\right)$. By Theorem 1.1 , we have $\tilde{u}_{n} \rightarrow u_{0}$ in $W^{1, p}\left(Q ; \mathbb{R}^{3}\right)$, and $\frac{1}{h_{n}} \partial_{3} \tilde{u}_{n} \rightarrow b_{0}$ in $L^{p}\left(Q ; \mathbb{R}^{3}\right)$. Setting $\varepsilon_{n}:=h_{n} / \gamma$, we claim that $\lim _{n} F_{\varepsilon_{n}}^{h_{n}}\left(\tilde{u}_{n}, Q\right)=K_{\gamma}^{\star}$. Indeed, it suffices to notice that

$$
\left|F_{\varepsilon_{n}}^{h_{n}}\left(\tilde{u}_{n}, Q\right)-F_{\tilde{\varepsilon}_{n}}^{h_{n}}\left(\tilde{u}_{n}, Q\right)\right| \leqslant\left(\left|1-\frac{\gamma \tilde{\varepsilon}_{n}}{h_{n}}\right|+\left|1-\frac{h_{n}}{\gamma \tilde{\varepsilon}_{n}}\right|\right) F_{\tilde{\varepsilon}_{n}}^{h_{n}}\left(\tilde{u}_{n}, Q\right) \underset{n \rightarrow \infty}{\longrightarrow} 0 .
$$

Extracting a further subsequence if necessary, we can find an exceptional set $Z \subset I$ of vanishing $\mathcal{H}^{1}$-measure such that for every $x_{2} \in I \backslash Z$, the slices $\tilde{u}_{n}\left(\cdot, x_{2}, \cdot\right)$ and $\frac{1}{h_{n}} \partial_{3} \tilde{u}_{n}\left(\cdot, x_{2}, \cdot\right)$ converge to $u_{0}$ in $W^{1, p}\left(I \times\left\{x_{2}\right\} \times I ; \mathbb{R}^{3}\right)$ and $b_{0}$ in $L^{p}\left(I \times\left\{x_{2}\right\} \times I ; \mathbb{R}^{3}\right)$ respectively. We select a level $s_{n} \in I \backslash Z$ satisfying

$$
\int_{I \times\left\{s_{n}\right\} \times I} \frac{1}{\varepsilon_{n}} W\left(\nabla_{h_{n}} \tilde{u}_{n}\right)+\varepsilon_{n}\left|\nabla_{h_{n}}^{2} \tilde{u}_{n}\right|^{2} d \mathcal{H}^{2} \leqslant F_{\varepsilon_{n}}^{h_{n}}\left(\tilde{u}_{n}, Q\right) .
$$


From now on we write

$$
u_{n}(x):=\tilde{u}_{n}\left(x_{1}, s_{n}, x_{3}\right) \text { and } \hat{u}_{n}\left(x_{1}, x_{3}\right):=\tilde{u}_{n}\left(x_{1}, s_{n}, x_{3}\right) .
$$

By our choice of $s_{n}$, we have $u_{n} \rightarrow u_{0}$ in $W^{1, p}\left(Q ; \mathbb{R}^{3}\right)$, and $\frac{1}{h_{n}} \partial_{3} u_{n} \rightarrow b_{0}$ in $L^{p}\left(Q ; \mathbb{R}^{3}\right)$. Since $\partial_{2} u_{n} \equiv 0$, assumption $\left(H_{4}\right)$ together with $(4.7)$ yields $\limsup _{n \rightarrow \infty} F_{\varepsilon_{n}}^{h_{n}}\left(u_{n}, Q\right) \leqslant \limsup _{n \rightarrow \infty} \int_{I \times\left\{s_{n}\right\} \times I} \frac{1}{\varepsilon_{n}} W\left(\nabla_{h_{n}} \tilde{u}_{n}\right)+\varepsilon_{n}\left|\nabla_{h_{n}}^{2} \tilde{u}_{n}\right|^{2} d \mathcal{H}^{2} \leqslant K_{\gamma}^{\star}$.

On the other hand, $\liminf { }_{n} F_{\varepsilon_{n}}^{h_{n}}\left(u_{n}, Q\right) \geqslant K_{\gamma}^{\star}$ by definition of $K_{\gamma}^{\star}$. Hence $\lim _{n} F_{\varepsilon_{n}}^{h_{n}}\left(u_{n}, Q\right)=K_{\gamma}^{\star}$.

Step 2 (first matching). We start partitioning $\left(\frac{1}{12}, \frac{1}{6}\right) \times Q^{\prime}$ into $M_{n}:=\left[\frac{1}{\varepsilon_{n}}\right]$ layers of width $\frac{1}{12 M_{n}}([\cdot]$ denotes the integer part). By Corollary 4.4, the energy concentrates near the interface $\left\{x_{1}=0\right\}$. Therefore we can find a layer $L_{n}:=$ $\left(\theta_{n}-\frac{1}{12 M_{n}}, \theta_{n}\right) \times Q^{\prime} \subset\left(\frac{1}{12}, \frac{1}{6}\right) \times Q^{\prime}$ for which

$$
\begin{aligned}
& M_{n}\left(\int_{L_{n}}\left|u_{n}-u_{0}\right|^{p}+\left|\frac{1}{h_{n}} \partial_{3} u_{n}-b_{0}\right|^{p}+\left|\nabla u_{n}-\nabla u_{0}\right|^{p} d x+F_{\varepsilon_{n}}^{h_{n}}\left(u_{n}, L_{n}\right)\right) \\
& \quad \leqslant \int_{\left(\frac{1}{12}, \frac{1}{6}\right) \times Q^{\prime}}\left|u_{n}-u_{0}\right|^{p}+\left|\frac{1}{h_{n}} \partial_{3} u_{n}-b_{0}\right|^{p}+\left|\nabla u_{n}-\nabla u_{0}\right|^{p} d x+F_{\varepsilon_{n}}^{h_{n}}\left(u_{n},\left(\frac{1}{12}, \frac{1}{6}\right) \times Q^{\prime}\right) \\
& \quad=: \alpha_{n} \rightarrow 0 .
\end{aligned}
$$

Then select a level $t_{n} \in\left(\theta_{n}-\frac{1}{12 M_{n}}, \theta_{n}\right)$ satisfying

$$
\begin{gathered}
\int_{\left\{t_{n}\right\} \times Q^{\prime}}\left|u_{n}-u_{0}\right|^{p}+\left|\frac{1}{h_{n}} \partial_{3} u_{n}-b_{0}\right|^{p}+\left|\nabla u_{n}-\nabla u_{0}\right|^{p} d \mathcal{H}^{2} \\
+\int_{\left\{t_{n}\right\} \times Q^{\prime}} \frac{1}{\varepsilon_{n}} W\left(\nabla_{h_{n}} u_{n}\right)+\varepsilon_{n}\left|\nabla_{h_{n}}^{2} u_{n}\right|^{2} d \mathcal{H}^{2} \leqslant 12 \alpha_{n} .
\end{gathered}
$$

Let $\varphi_{n} \in C^{\infty}(\mathbb{R})$ be a cut-off function satisfying

$$
\left\{\begin{array}{l}
0 \leqslant \varphi_{n} \leqslant 1 \\
\varphi_{n}(t)=1 \text { for } t \leqslant \theta_{n}-\frac{1}{12 M_{n}} \\
\varphi_{n}(t)=0 \text { for } t \geqslant \theta_{n}, \\
\varepsilon_{n}\left|\varphi_{n}^{\prime}\right|+\varepsilon_{n}^{2}\left|\varphi_{n}^{\prime \prime}\right| \leqslant C,
\end{array}\right.
$$

for a constant $C$ independent of $n$. For $x \in L_{n}$, we set

$$
v_{n}(x):=\left(1-\varphi_{n}\left(x_{1}\right)\right)\left(u_{0}(x)+h_{n} x_{3} b_{0}(x)+\bar{u}_{n}\left(x_{3}\right)\right)+\varphi_{n}\left(x_{1}\right) u_{n}(x),
$$

with

$$
\bar{u}_{n}\left(x_{3}\right):=\hat{u}_{n}\left(t_{n}, x_{3}\right)-\bar{u}_{0}\left(t_{n}\right)-x_{3} \int_{I} \partial_{3} \hat{u}_{n}\left(t_{n}, s\right) d s .
$$

We claim that

$$
\begin{aligned}
& \int_{L_{n}}\left|v_{n}-u_{0}\right|^{p} d x \rightarrow 0 \\
& \frac{1}{\varepsilon_{n}} \int_{L_{n}}\left|\frac{1}{h_{n}} \partial_{3} v_{n}-b_{0}\right|^{p} d x \rightarrow 0
\end{aligned}
$$




$$
\begin{aligned}
& \frac{1}{\varepsilon_{n}} \int_{L_{n}}\left|\nabla^{\prime} v_{n}-\nabla^{\prime} u_{0}\right|^{p} d x \rightarrow 0, \\
& \frac{1}{\varepsilon_{n}} \int_{L_{n}} W\left(\nabla_{h_{n}} v_{n}\right) d x \rightarrow 0, \\
& \varepsilon_{n} \int_{L_{n}}\left|\nabla_{h_{n}}^{2} v_{n}\right|^{2} d x \rightarrow 0 .
\end{aligned}
$$

Applying Jensen's inequality, we derive from (4.9) that

$$
\int_{I}\left|\bar{u}_{n}\right|^{p} d x_{3} \leqslant C \int_{\left\{t_{n}\right\} \times Q^{\prime}}\left|u_{n}-u_{0}\right|^{p}+\left|\partial_{3} u_{n}\right|^{p} d \mathcal{H}^{2} \leqslant C \alpha_{n} \rightarrow 0 .
$$

Then (4.11) easily follows from (4.8), (4.9), and (4.16). To prove (4.12), we first estimate for $x_{3} \in I$,

$$
\left|\bar{u}_{n}^{\prime}\left(x_{3}\right)\right| \leqslant \int_{I}\left|\partial_{33}^{2} \hat{u}_{n}\left(t_{n}, s\right)\right| d s \leqslant\left(\int_{\left\{t_{n}\right\} \times Q^{\prime}}\left|\partial_{33}^{2} u_{n}\right|^{2} d \mathcal{H}^{2}\right)^{1 / 2} \leqslant C \alpha_{n}^{1 / 2} \varepsilon_{n}^{3 / 2},
$$

where we have used Poincaré's inequality, Hölder's inequality, and (4.9). We may now infer that

$$
\begin{aligned}
& \frac{1}{\varepsilon_{n}} \int_{L_{n}}\left|\frac{1}{h_{n}} \partial_{3} v_{n}-b_{0}\right|^{p} d x \leqslant C\left(\frac{1}{\varepsilon_{n}} \int_{L_{n}}\left|\frac{1}{h_{n}} \partial_{3} u_{n}-b_{0}\right|^{p} d x+\frac{1}{\varepsilon_{n}^{p}} \int_{I}\left|\bar{u}_{n}^{\prime}\right|^{p} d x_{3}\right) \\
& \quad \leqslant C \alpha_{n} \rightarrow 0
\end{aligned}
$$

thanks to (4.8) and (4.17). Observing that

$$
u_{n}-u_{0}-h_{n} x_{3} b_{0}-\bar{u}_{n}=x_{3} \int_{\left\{t_{n}\right\} \times Q^{\prime}}\left(\partial_{3} u_{n}-h_{n} b_{0}\right) d \mathcal{H}^{2} \quad \text { on }\left\{t_{n}\right\} \times Q^{\prime},
$$

we can apply Poincaré's inequality to obtain

$$
\begin{aligned}
& \int_{L_{n}}\left|u_{n}-u_{0}-h_{n} x_{3} b_{0}-\bar{u}_{n}\right|^{p} d x \leqslant \frac{C}{M_{n}} \int_{\left\{t_{n}\right\} \times Q^{\prime}}\left|\partial_{3} u_{n}-h_{n} b_{0}\right|^{p} d \mathcal{H}^{2} \\
& \quad+C\left(\frac{1}{M_{n}}\right)^{p} \int_{L_{n}}\left|\partial_{1} u_{n}-\partial_{1} u_{0}\right|^{p} d x \leqslant C \alpha_{n} \varepsilon_{n}^{p+1} .
\end{aligned}
$$

Then, using (4.8)-(4.10), and (4.18) we derive

$$
\begin{aligned}
& \frac{1}{\varepsilon_{n}} \int_{L_{n}}\left|\nabla^{\prime} v_{n}-\nabla^{\prime} u_{0}\right|^{p} d x \leqslant \frac{C}{\varepsilon_{n}} \int_{L_{n}}\left(\frac{1}{\varepsilon_{n}^{p}}\left|u_{n}-u_{0}-h_{n} x_{3} b_{0}-\bar{u}_{n}\right|^{p}+\left|\nabla^{\prime} u_{n}-\nabla^{\prime} u_{0}\right|^{p}\right) d x \\
& \quad \leqslant C \alpha_{n} \rightarrow 0,
\end{aligned}
$$

and (4.13) is proved. In view of (4.5), estimate (4.14) follows from (4.12) and (4.13), i.e.,

$$
\begin{aligned}
\frac{1}{\varepsilon_{n}} \int_{L_{n}} W\left(\nabla_{h_{n}} v_{n}\right) d x & \leqslant \frac{C_{\star}}{\varepsilon_{n}} \int_{L_{n}} \min \left(\left|\nabla_{h_{n}} v_{n}-A\right|^{p},\left|\nabla_{h_{n}} v_{n}-B\right|^{p}\right) d x \\
& \leqslant \frac{C_{\star}}{\varepsilon_{n}} \int_{L_{n}}\left|\nabla_{h_{n}} v_{n}-\left(\nabla^{\prime} u_{0}, b_{0}\right)\right|^{p} d x \rightarrow 0
\end{aligned}
$$


We prove (4.15) in separate parts. In view of (4.10) we have

$$
\begin{aligned}
& \varepsilon_{n} \int_{L_{n}}\left|\left(\nabla^{\prime}\right)^{2} v_{n}\right|^{2} d x \leqslant C\left(\varepsilon_{n} \int_{L_{n}}\left|\left(\nabla^{\prime}\right)^{2} u_{n}\right|^{2} d x+\frac{1}{\varepsilon_{n}} \int_{L_{n}}\left|\nabla^{\prime} u_{n}-\nabla^{\prime} u_{0}\right|^{2} d x\right. \\
& \left.\quad+\frac{1}{\varepsilon_{n}^{3}} \int_{L_{n}}\left|u_{n}-u_{0}-h_{n} x_{3} b_{0}-\bar{u}_{n}\right|^{2} d x\right),
\end{aligned}
$$

and we shall estimate each term separately. The first term on the right-handside of the inequality converges to 0 by (4.8). For the last two terms, we use (4.8) and (4.18) together with Hölder's inequality to obtain

$$
\frac{1}{\varepsilon_{n}} \int_{L_{n}}\left|\nabla^{\prime} u_{n}-\nabla^{\prime} u_{0}\right|^{2} d x \leqslant \frac{\left|L_{n}\right|^{\frac{p-2}{p}}}{\varepsilon_{n}}\left(\int_{L_{n}}\left|\nabla^{\prime} u_{n}-\nabla^{\prime} u_{0}\right|^{p} d x\right)^{2 / p} \leqslant C \alpha_{n}^{2 / p} \rightarrow 0
$$

and

$$
\begin{aligned}
& \frac{1}{\varepsilon_{n}^{3}} \int_{L_{n}}\left|u_{n}-u_{0}-h_{n} x_{3} b_{0}-\bar{u}_{n}\right|^{2} d x \leqslant \frac{\left|L_{n}\right|^{\frac{p-2}{p}}}{\varepsilon_{n}^{3}}\left(\int_{L_{n}}\left|u_{n}-u_{0}-h_{n} x_{3} b_{0}-\bar{u}_{n}\right|^{p} d x\right)^{2 / p} \\
& \quad \leqslant C \alpha_{n}^{2 / p} \rightarrow 0,
\end{aligned}
$$

and we conclude that $\varepsilon_{n} \int_{L_{n}}\left|\left(\nabla^{\prime}\right)^{2} v_{n}\right|^{2} d x \rightarrow 0$. Finally we estimate

$$
\begin{aligned}
& \varepsilon_{n} \int_{L_{n}}\left|\nabla^{\prime}\left(\frac{1}{h_{n}} \partial_{3} v_{n}\right)\right|^{2} d x \leqslant C\left(\varepsilon_{n} \int_{L_{n}}\left|\nabla^{\prime}\left(\frac{1}{h_{n}} \partial_{3} u_{n}\right)\right|^{2} d x\right. \\
& \left.\quad+\frac{1}{\varepsilon_{n}} \int_{L_{n}}\left|\frac{1}{h_{n}} \partial_{3} u_{n}-b_{0}\right|^{2} d x+\frac{1}{\varepsilon_{n}^{2}} \int_{I}\left|\bar{u}_{n}^{\prime}\right|^{2} d x_{3}\right) \leqslant C\left(\alpha_{n}+\alpha_{n}^{2 / p}\right) \rightarrow 0,
\end{aligned}
$$

where we have used again (4.10), Hölder's inequality, (4.8), and (4.17). Since $\bar{u}_{n}^{\prime \prime}\left(x_{3}\right)=\partial_{33}^{2} \hat{u}_{n}\left(t_{n}, x_{3}\right)$, we infer from $(4.8)-(4.10)$ that

$$
\begin{aligned}
& \varepsilon_{n} \int_{L_{n}} \frac{1}{h_{n}^{4}}\left|\partial_{33}^{2} v_{n}\right|^{2} d x \leqslant C\left(\frac{\varepsilon_{n}}{h_{n}^{4}} \int_{L_{n}}\left|\partial_{33}^{2} u_{n}\right|^{2} d x+\frac{\varepsilon_{n}^{2}}{h_{n}^{4}} \int_{\left\{t_{n}\right\} \times Q^{\prime}}\left|\partial_{33}^{2} u_{n}\right|^{2} d \mathcal{H}^{2}\right) \\
& \quad \leqslant C \varepsilon_{n} \alpha_{n} \rightarrow 0,
\end{aligned}
$$

which ends the proof of (4.15).

Step 3 (second matching). Let $\psi_{n} \in C^{\infty}(\mathbb{R})$ be such that $0 \leqslant \psi_{n} \leqslant$ $1, \psi_{n}(t)=1$ if $t \leqslant \theta_{n}, \psi_{n}(t)=0$ if $t \geqslant 1 / 4$, and $\left|\psi_{n}^{\prime}\right|+\left|\psi_{n}^{\prime \prime}\right| \leqslant C$ for a constant $C$ independent of $n$. For $x \in\left(\theta_{n}, \frac{1}{4}\right) \times Q^{\prime}$, we set

$$
w_{n}(x):=u_{0}(x)+h_{n} x_{3} b_{0}(x)+c_{n}^{+}+\psi_{n}\left(x_{1}\right)\left(\bar{u}_{n}\left(x_{3}\right)-c_{n}^{+}\right),
$$

where $c_{n}^{+}:=\int_{I} \bar{u}_{n}\left(x_{3}\right) d x_{3} \rightarrow 0$ thanks to (4.16). We claim that

$$
\begin{aligned}
& \int_{\left(\theta_{n}, \frac{1}{4}\right) \times Q^{\prime}}\left|w_{n}-u_{0}\right|^{p} d x \rightarrow 0, \\
& \frac{1}{\varepsilon_{n}} \int_{\left(\theta_{n}, \frac{1}{4}\right) \times Q^{\prime}}\left|\frac{1}{h_{n}} \partial_{3} w_{n}-b_{0}\right|^{p} d x \rightarrow 0, \\
& \frac{1}{\varepsilon_{n}} \int_{\left(\theta_{n}, \frac{1}{4}\right) \times Q^{\prime}} W\left(\nabla_{h_{n}} w_{n}\right) d x \rightarrow 0,
\end{aligned}
$$


Vol. 20 (2013)

$$
\varepsilon_{n} \int_{\left(\theta_{n}, \frac{1}{4}\right) \times Q^{\prime}}\left|\nabla_{h_{n}}^{2} w_{n}\right|^{2} d x \rightarrow 0 .
$$

First, (4.20) and (4.21) are easy consequences of (4.9) and (4.17) respectively. Next we apply Poincaré's inequality and (4.17) to derive that

$$
\begin{aligned}
& \frac{1}{\varepsilon_{n}} \int_{\left(\theta_{n}, \frac{1}{4}\right) \times Q^{\prime}}\left|\nabla^{\prime} w_{n}-\nabla^{\prime} u_{0}\right|^{p} d x \leqslant \frac{C}{\varepsilon_{n}} \int_{I}\left|\bar{u}_{n}-c_{n}^{+}\right|^{p} d x_{3} \leqslant \frac{C}{\varepsilon_{n}} \int_{I}\left|\bar{u}_{n}^{\prime}\right|^{p} d x_{3} \\
& \quad \leqslant C \varepsilon_{n}^{\frac{3 p-2}{2}} \alpha_{n}^{\frac{p}{2}} \rightarrow 0
\end{aligned}
$$

Then, to prove (4.22) we argue exactly as in (4.19) using (4.21) and (4.24). We finally obtain in a similar way that

$$
\begin{aligned}
& \varepsilon_{n} \int_{\left(\theta_{n}, \frac{1}{4}\right) \times Q^{\prime}}\left|\nabla_{h_{n}}^{2} w_{n}\right|^{2} d x \\
& \quad \leqslant C\left(\varepsilon_{n} \int_{\left(\theta_{n}, \frac{1}{4}\right) \times Q^{\prime}}\left|\bar{u}_{n}-c_{n}^{+}\right|^{2} d x+\frac{1}{\varepsilon_{n}} \int_{I}\left|\bar{u}_{n}^{\prime}\right|^{2} d x_{3}+\frac{\varepsilon_{n}}{h_{n}^{4}} \int_{\left\{t_{n}\right\} \times Q^{\prime}}\left|\partial_{33}^{2} u_{n}\right|^{2} d \mathcal{H}^{2}\right) \\
& \quad \leqslant C \alpha_{n} \rightarrow 0,
\end{aligned}
$$

and (4.23) is proved.

Step 4. To conclude the proof, we first set for $x \in Q$,

$$
g_{n}^{+}(x):= \begin{cases}u_{n}(x) & \text { for } x_{1}<\theta_{n}-\frac{1}{12 M_{n}}, \\ v_{n}(x) & \text { for } \theta_{n}-\frac{1}{12 M_{n}} \leqslant x_{1}<\theta_{n}, \\ w_{n}(x) & \text { for } \theta_{n} \leqslant x_{1}<\frac{1}{4}, \\ u_{0}(x)+h_{n} x_{3} b_{0}(x)+c_{n}^{+} & \text {for } \frac{1}{4} \leqslant x_{1} \leqslant \frac{1}{2} .\end{cases}
$$

Recalling that $h_{n}=\gamma \varepsilon_{n}$, it follows from the previous steps and Corollary 4.4 that $g_{n}^{+} \in C^{2}\left(Q ; \mathbb{R}^{3}\right), g_{n}^{+} \rightarrow u_{0}$ in $W^{1, p}\left(Q ; \mathbb{R}^{3}\right), \frac{1}{\gamma \varepsilon_{n}} \partial_{3} g_{n}^{+} \rightarrow b_{0}$ in $L^{p}\left(Q ; \mathbb{R}^{3}\right)$, and $\lim _{n} F_{\varepsilon_{n}}^{\gamma \varepsilon_{n}}\left(g_{n}^{+}, Q\right)=\lim _{n} F_{\varepsilon_{n}}^{\gamma \varepsilon_{n}}\left(u_{n}, Q\right)=K_{\gamma}^{\star}$. The sequence $\left\{g_{n}^{+}\right\}$satisfies the pinning condition $g_{n}^{+}=u_{0}+\gamma \varepsilon_{n} x_{3} b_{0}+c_{n}^{+}$in $Q \cap\left\{x_{1}>1 / 4\right\}$. Then we repeat construction to modify $g_{n}^{+}$in $\left(-\frac{1}{2}, 0\right) \times Q^{\prime}$ in order build a new field $g_{n}^{-} \in C^{2}\left(Q ; \mathbb{R}^{3}\right)$ satisfying $g_{n}^{-}=u_{0}+\gamma \varepsilon_{n} x_{3} b_{0}+c_{n}^{-}$in $Q \cap\left\{x_{1}<-1 / 4\right\}$ for some constants $c_{n}^{-} \rightarrow 0$. Now it suffices to set $g_{n}:=g_{n}^{-}-c_{n}^{+}$and $c_{n}:=c_{n}^{-}-c_{n}^{+}$. By construction $g_{n}$ does not dependent on $x_{2}$, that is $g_{n}(x)=: \hat{g}_{n}\left(x_{1}, x_{3}\right)$.

Corollary 4.10. Assume that $\left(H_{1}\right)-\left(H_{4}\right)$ and (1.5) hold. Then $K_{\gamma}^{\star} \geqslant K_{\gamma}$.

Proof. We consider the sequences $\left\{\varepsilon_{n}\right\}$ and $\left\{g_{n}\right\}$ given by Proposition 4.9. Remind that $g_{n}(x)=\hat{g}_{n}\left(x_{1}, x_{3}\right)$. We set $\ell_{n}:=1 / \varepsilon_{n}$, and for $y=\left(y_{1}, y_{2}\right) \in$ $\ell_{n} I \times \gamma I, v_{n}(y):=\frac{1}{\varepsilon_{n}} \hat{g}_{n}\left(\varepsilon_{n} y_{1}, y_{2} / \gamma\right)$. Then straightforward computations yield $\nabla v_{n}(y)=\left(\bar{u}_{0}^{\prime}\left(y_{1}\right), \bar{b}_{0}\left(y_{1}\right)\right)$ nearby $\left\{\left|y_{1}\right|=\ell_{n} / 2\right\}$, and

$$
K_{\gamma} \leqslant \frac{1}{\gamma} \int_{\ell_{n} I \times \gamma I} \mathcal{W}\left(\nabla v_{n}\right)+\left|\nabla^{2} v_{n}\right|^{2} d y=F_{\varepsilon_{n}}^{\gamma \varepsilon_{n}}\left(g_{n}, Q\right) .
$$

By construction of $\left\{g_{n}\right\}$, the conclusion follows letting $n \rightarrow \infty$. 


\subsection{The $\Gamma$ - lim sup inequality}

We conclude this section with the construction of a recovery sequence. Then Theorem 4.11 together with Corollary 4.10 and Theorem 4.6 concludes the proof of Theorem 1.2.

Theorem 4.11. Assume that $\left(H_{1}\right)-\left(H_{5}\right)$ and (1.5) hold. Let $\varepsilon_{n} \rightarrow 0$ and $h_{n} \rightarrow 0$ be arbitrary sequences such that $h_{n} / \varepsilon_{n} \rightarrow \gamma$. Then, for every $(u, b) \in \mathscr{C}$, there exists a sequence $\left\{u_{n}\right\} \subset H^{2}\left(\Omega ; \mathbb{R}^{3}\right)$ such that $u_{n} \rightarrow u$ in $W^{1, p}\left(\Omega ; \mathbb{R}^{3}\right), \frac{1}{h_{n}} \partial_{3} u_{n} \rightarrow b$ in $L^{p}\left(\Omega ; \mathbb{R}^{3}\right)$ and

$$
\lim _{n \rightarrow \infty} F_{\varepsilon_{n}}^{h_{n}}\left(u_{n}\right)=K_{\gamma} \operatorname{Per}_{\omega}(E),
$$

where $\left(\nabla^{\prime} u, b\right)(x)=\left(1-\chi_{E}\left(x^{\prime}\right)\right) A+\chi_{E}\left(x^{\prime}\right) B$.

Proof. Step 1. We first assume that $A^{\prime} \neq B^{\prime}$, so that $\partial^{*} E \cap \omega$ is of the form (2.5) by Theorem 2.1. We also assume that it is made by finitely many interfaces, i.e., $\mathscr{I}=\{1, \ldots, m\}$ in (2.5). In this case, by Theorem 2.1, we have $u(x)=\bar{u}\left(x_{1}\right)$ and $b(x)=\bar{b}\left(x_{1}\right)$ where $\bar{u} \in W^{1, \infty}\left(\left(\alpha_{\min }, \alpha_{\max }\right) ; \mathbb{R}^{3}\right),\left(\bar{u}^{\prime}, \bar{b}\right) \in$ $B V\left(\left(\alpha_{\min }, \alpha_{\max }\right) ;\left\{\left(a, A_{3}\right),\left(-a,-A_{3}\right)\right\}\right)$, and $\left(\alpha_{\min }, \alpha_{\max }\right)$ is defined by $(2.3)$. Without loss of generality we may assume that $\bar{u}^{\prime}\left(x_{1}\right)=-a$ for $x_{1}<\alpha_{1}$. Then $\bar{u}^{\prime}(t)=a$ for $\alpha_{i}<t<\alpha_{i+1}$ if $i$ is odd, $\bar{u}^{\prime}(t)=-a$ for $\alpha_{i}<t<\alpha_{i+1}$ if $i$ is even, and $\bar{u}^{\prime}(t)=a$ or $\bar{u}^{\prime}(t)=-a$ for $t>\alpha_{m}$ if $m$ is odd or even respectively.

Let us consider for each $k \in \mathbb{N}$, some $\ell_{k}>0$ and $v_{k} \in C^{2}\left(\ell_{k} I \times \gamma I ; \mathbb{R}^{3}\right)$ such that $\nabla v_{k}(y)=\left(\bar{u}_{0}^{\prime}, \bar{b}_{0}\right)\left(y_{1}\right)$ nearby $\left\{\left|y_{1}\right|=\ell_{k} / 2\right\}$, and

$$
\frac{1}{\gamma} \int_{\ell_{k} I \times \gamma I} \mathcal{W}\left(\nabla v_{k}\right)+\left|\nabla^{2} v_{k}\right|^{2} d y \leqslant K_{\gamma}+2^{-k} .
$$

Subtracting a constant to $v_{k}$ if necessary, we may assume that

$$
v_{k}(y)= \begin{cases}a y_{1}+A_{3} y_{2}+c_{k} & \text { nearby }\left\{y_{1}=\ell_{k} / 2\right\}, \\ -a y_{1}+B_{3} y_{2}-c_{k} & \text { nearby }\left\{y_{1}=-\ell_{k} / 2\right\},\end{cases}
$$

for some $c_{k} \in \mathbb{R}^{3}$.

Let $h_{n} \rightarrow 0$ be an arbitrary sequence, and without loss of generality we can choose $\varepsilon_{n}:=h_{n} / \gamma($ see $(4.6))$. We fix for each $i=1, \ldots, m$, a bounded open interval $J_{i}^{\prime} \subset \mathbb{R}$ such that

$$
J_{i} \subset \subset J_{i}^{\prime} \text { and } \mathcal{H}^{1}\left(J_{i}^{\prime} \backslash J_{i}\right) \leqslant 2^{-k},
$$

and we shall consider integers $n$ large enough in such a way that $\alpha_{i}+\ell_{k} \varepsilon_{n} / 2<$ $\alpha_{i+1}-\ell_{k} \varepsilon_{n} / 2$ for every $i=1, \ldots, m-1$. We write for each $i=1, \ldots, m$,

$$
\alpha_{i-}^{n}:=\alpha_{i}-\frac{\ell_{k} \varepsilon_{n}}{2} \quad \text { and } \quad \alpha_{i+}^{n}:=\alpha_{i}+\frac{\ell_{k} \varepsilon_{n}}{2} .
$$

Note that by convexity of $\omega$,

$$
\left(\left(\alpha_{i-}^{n}, \alpha_{i+}^{n}\right) \times \mathbb{R}\right) \cap \omega \subset\left(\alpha_{i-}^{n}, \alpha_{i+}^{n}\right) \times J_{i}^{\prime}
$$

whenever $n$ is sufficiently large. 
We define the transition layer near each interface as follows: for each $i=1, \ldots, m$, we set for $x \in\left(\alpha_{i-}^{n}, \alpha_{i+}^{n}\right) \times J_{i}^{\prime} \times I$,

$w_{n, k}^{i}(x):=(-1)^{i+1} v_{k}\left((-1)^{i+1} \frac{x_{1}-\alpha_{i}}{\varepsilon_{n}},(-1)^{i+1} \gamma x_{3}\right)+\left(1+(-1)^{i}\right)\left(a \frac{\ell_{k}}{2}+c_{k}\right)$.

Observe that (4.28) yields

$$
w_{k, n}^{i}\left(\alpha_{i-}^{n}, x_{2}, x_{3}\right)=v_{k}\left((-1)^{i} \frac{\ell_{k}}{2}, \gamma x_{3}\right),
$$

and

$$
w_{k, n}^{i}\left(\alpha_{i+}^{n}, x_{2}, x_{3}\right)=v_{k}\left((-1)^{i+1} \frac{\ell_{k}}{2}, \gamma x_{3}\right)+2\left(1+(-1)^{i}\right) c_{k} .
$$

Setting

$$
\beta_{i}^{n}:=\sum_{j=1}^{i} \bar{u}\left(\alpha_{j+}^{n}\right)-\bar{u}\left(\alpha_{j-}^{n}\right) \quad \text { and } \quad \kappa_{i}:=2 \sum_{j=1}^{i}\left(1+(-1)^{j}\right),
$$

with $\beta_{0}^{n}:=0, \kappa_{0}:=0$, we finally define for $n$ large enough and $x \in \Omega$,

$u_{n, k}(x):= \begin{cases}\bar{u}\left(x_{1}\right)+\varepsilon_{n} v_{k}\left(-\frac{\ell_{k}}{2}, \gamma x_{3}\right) & \text { for } x_{1} \leqslant \alpha_{1-}^{n}, \\ \bar{u}\left(\alpha_{i-}^{n}\right)-\beta_{i-1}^{n}+\varepsilon_{n} w_{k, n}^{i}(x)+\varepsilon_{n} \kappa_{i-1} c_{k} & \text { for } \alpha_{i-}^{n}<x_{1}<\alpha_{i+}^{n}, \\ \bar{u}\left(x_{1}\right)-\beta_{i}^{n}+\varepsilon_{n} v_{k}\left((-1)^{i+1} \frac{\ell_{k}}{2}, \gamma x_{3}\right)+\varepsilon_{n} \kappa_{i} c_{k} & \text { for } \alpha_{i+}^{n} \leqslant x_{1} \leqslant \alpha_{(i+1)-}^{n}, \\ \bar{u}\left(x_{1}\right)-\beta_{m}^{n}+\varepsilon_{n} v_{k}\left((-1)^{m+1} \frac{\ell_{k}}{2}, \gamma x_{3}\right)+\varepsilon_{n} \kappa_{m} c_{k} & \text { for } x_{1} \geqslant \alpha_{m+}^{n} .\end{cases}$

In view of $(4.32)-(4.33)$ we have $u_{n, k} \in H^{2}\left(\Omega ; \mathbb{R}^{3}\right)$. Moreover, $u_{n, k}$ does not depend on the $x_{2}$-variable, and

$$
\left(\partial_{1} u_{n, k}, \frac{1}{h_{n}} \partial_{3} u_{n, k}\right)(x)= \begin{cases}\nabla v_{k}\left((-1)^{i+1} \frac{x_{1}-\alpha_{i}}{\varepsilon_{n}},(-1)^{i+1} \gamma x_{3}\right) & \text { if } \alpha_{i-}^{n}<x_{1}<\alpha_{i+}^{n}, \\ \left(\bar{u}^{\prime}, \bar{b}\right)\left(x_{1}\right) & \text { otherwise. }\end{cases}
$$

Since $\bar{u}$ is Lipschitz continuous, we have $\left|\beta_{i}^{n}\right| \leqslant C \varepsilon_{n}$ for a constant $C$ independent of $n$. In addition, $v_{k}$ and $\nabla v_{k}$ are bounded, and we infer that $u_{n, k} \rightarrow u$ in $W^{1, p}\left(\Omega ; \mathbb{R}^{3}\right)$ and $\frac{1}{h_{n}} \partial_{3} u_{n, k} \rightarrow b$ in $L^{p}\left(\Omega ; \mathbb{R}^{3}\right)$ as $n \rightarrow \infty$. Using (4.31), (4.35), and changing variables, we estimate

$$
\begin{aligned}
F_{\varepsilon_{n}}^{h_{n}}\left(u_{n, k}\right) & \leqslant \sum_{i=1}^{m} F_{\varepsilon_{n}}^{h_{n}}\left(\varepsilon_{n} w_{n, k}^{i},\left(\alpha_{i-}^{n}, \alpha_{i+}^{n}\right) \times J_{i}^{\prime} \times I\right) \\
& \leqslant \sum_{i=1}^{m} \frac{\mathcal{H}^{1}\left(J_{i}^{\prime}\right)}{\gamma} \int_{\ell_{k} I \times \gamma I} \mathcal{W}\left(\nabla v_{k}\right)+\left|\nabla^{2} v_{k}\right|^{2} d y \\
& \leqslant K_{\gamma} \operatorname{Per}_{\omega}(E)+C_{0} 2^{-k},
\end{aligned}
$$

for a constant $C_{0}$ which only depends on $m$ and $\operatorname{Per}_{\omega}(E)$. 
For each $k \in \mathbb{N}$, we can now find $N_{k} \in \mathbb{N}$ such that

$$
\begin{gathered}
\left\|u_{n, k}-u\right\|_{W^{1, p}(\Omega)} \leqslant 2^{-k}, \quad\left\|\frac{1}{h_{n}} \partial_{3} u_{n, k}-b\right\|_{L^{p}(\Omega)} \leqslant 2^{-k}, \\
F_{\varepsilon_{n}}^{h_{n}}\left(u_{n, k}\right) \leqslant K_{\gamma} \operatorname{Per}_{\omega}(E)+C_{0} 2^{-k}
\end{gathered}
$$

for every $n \geqslant N_{k}$. Moreover we can assume that the resulting sequence $\left\{N_{k}\right\}$ satisfies $N_{k}<N_{k+1}$ for every $k \in \mathbb{N}$. Then for every $n \geqslant N_{0}$, there exists a unique $k_{n}$ such that $N_{k_{n}} \leqslant n<N_{k_{n}+1}$, and $k_{n} \rightarrow+\infty$ as $n \rightarrow+\infty$. We define $u_{n}:=u_{n, k_{n}}$ and it follows that $u_{n} \rightarrow u$ in $W^{1, p}\left(\Omega ; \mathbb{R}^{3}\right), \frac{1}{h_{n}} \partial_{3} u_{n} \rightarrow b$ in $L^{p}\left(\Omega ; \mathbb{R}^{3}\right)$,

$$
\limsup _{n \rightarrow \infty} F_{\varepsilon_{n}}^{h_{n}}\left(u_{n}\right) \leqslant K_{\gamma} \operatorname{Per}_{\omega}(E) .
$$

Finally (4.26) holds by (4.36), Theorem 4.6, and Corollary 4.10.

Step 2. We now consider the case where $A^{\prime} \neq B^{\prime}$ and $\partial^{*} E \cap \omega$ is made by infinitely many interfaces, i.e., $\partial^{*} E \cap \omega$ is as in (2.5) with $\mathscr{I} \subset \mathbb{Z}$ infinite. We may assume for simplicity that $\mathscr{I}=\mathbb{N}$. The general case can be recovered from the discussion below with the obvious modifications.

By Theorem 2.1, we have $\lim _{k} \sum_{i=0}^{k} \mathcal{H}^{1}\left(J_{i}\right)=\sum_{i \in \mathbb{N}} \mathcal{H}^{1}\left(J_{i}\right)=\operatorname{Per}_{\omega}(E)$, and $\alpha_{k}$ converges to $\alpha_{\max }$. For $k \in \mathbb{N}$ large enough, we define some $u_{k} \in$ $W^{1, \infty}\left(\Omega ; \mathbb{R}^{3}\right)$ in the following way: we set $u_{k}(x):=u(x)$ for $x \in \Omega \cap\left\{x_{1}<\right.$ $\left.\alpha_{k+1}\right\}$, and we extend $u_{k}$ to be affine in the remaining of $\Omega$ in such a way that $u_{k}$ and $\nabla u_{k}$ are continuous across the interface $\left\{x_{1}=\alpha_{k+1}\right\}$. Similarly we define for $x \in \Omega \cap\left\{x_{1}<\alpha_{k+1}\right\}, b_{k}(x):=b(x)$, and we extend $b_{k}$ by a suitable constant in the remaining of $\Omega$ so that it remains continuous across $\left\{x_{1}=\alpha_{k+1}\right\}$. Then one may check that $\left(u_{k}, b_{k}\right) \in \mathscr{C}$, and that $\left(\nabla^{\prime} u_{k}, b_{k}\right)=$ $\left(1-\chi_{E_{k}}\left(x^{\prime}\right)\right) A+\chi_{E_{k}}\left(x^{\prime}\right) B$ with $\partial^{*} E_{k} \cap \omega=\bigcup_{i=0}^{k}\left\{\alpha_{i}\right\} \times J_{i}$. Moreover, using the fact that $\alpha_{k} \rightarrow \alpha_{\max }$, we derive that $u_{k} \rightarrow u$ in $W^{1, p}\left(\Omega ; \mathbb{R}^{3}\right)$ and $b_{k} \rightarrow b$ in $L^{p}\left(\Omega ; \mathbb{R}^{3}\right)$.

Let $h_{n} \rightarrow 0$ and $\varepsilon_{n} \rightarrow 0$ be arbitrary sequences such that $h_{n} / \varepsilon_{n} \rightarrow \gamma$. Since $\partial^{*} E_{k} \cap \omega$ is made by finitely many interfaces, by Step 1 , we can find $\left\{u_{n, k}\right\} \subset H^{2}\left(\Omega ; \mathbb{R}^{3}\right)$ such that $u_{n, k} \rightarrow u_{k} \operatorname{in} W^{1, p}\left(\Omega \mathbb{R}^{3}\right), \frac{1}{h_{n}} \partial_{3} u_{n, k} \rightarrow b_{k}$ in $L^{p}\left(\Omega ; \mathbb{R}^{3}\right)$, and $\lim _{n} F_{\varepsilon_{n}}^{h_{n}}\left(u_{n, k}\right)=K_{\gamma} \sum_{i=0}^{k} \mathcal{H}^{1}\left(J_{i}\right)$. Then the conclusion follows for a suitable diagonal sequence $u_{n}:=u_{n, k_{n}}$ as already pursued in Step 1.

Step 3. We finally treat the case $A^{\prime}=B^{\prime}[=0$ by (1.5)]. Without loss of generality we may assume that $u=0$. According to Theorem 2.1, we have $b(x)=\left(1-\chi_{E}\left(x^{\prime}\right)\right) A_{3}+\chi_{E}\left(x^{\prime}\right) B_{3}$ where $E \subset \omega$ is a set of finite perimeter in $\omega$. By Lemma 4.3 in [3], we can find a sequence $\left\{E_{k}\right\}$ of bounded open sets in $\mathbb{R}^{2}$ with smooth boundary such that $\chi_{E_{k}} \rightarrow \chi_{E}$ in $L^{1}(\omega)$, and $\lim _{k} \mathcal{H}^{1}\left(\partial E_{k} \cap \bar{\omega}\right)=$ $\operatorname{Per}_{\omega}(E)$. We define for $x \in \Omega, b_{k}(x):=\left(1-\chi_{E_{k}}\left(x^{\prime}\right)\right) A_{3}+\chi_{E_{k}}\left(x^{\prime}\right) B_{3}$, so that $b_{k} \rightarrow b$ in $L^{p}\left(\Omega ; \mathbb{R}^{3}\right)$. Since $\mathcal{M}^{k}:=\partial E_{k}$ is a smooth submanifold of $\mathbb{R}^{2}$, for every $k \in \mathbb{N}$ we can find $\delta_{k}>0$ such that the nearest point projection onto $\mathcal{M}^{k}$ is well defined and smooth in the tubular $\delta_{k}$-neighborhood

$$
U_{k}:=\left\{x^{\prime} \in \mathbb{R}^{2}: \operatorname{dist}\left(x^{\prime}, \mathcal{M}^{k}\right)<\delta_{k}\right\}
$$


We define the signed distance to $\mathcal{M}^{k}$ as the function $d_{k}: \mathbb{R}^{2} \rightarrow[0,+\infty)$ given by

$$
d_{k}\left(x^{\prime}\right):= \begin{cases}-\operatorname{dist}\left(x^{\prime}, \mathcal{M}^{k}\right) & \text { if } x \in E_{k} \\ \operatorname{dist}\left(x^{\prime}, \mathcal{M}^{k}\right) & \text { otherwise. }\end{cases}
$$

Then $d_{k}$ is smooth in $U_{k}$, the level sets $\left\{d_{k}=t\right\}=: \mathcal{M}_{t}^{k}$ are smooth for all $t \in\left(-\delta_{k}, \delta_{k}\right)$, and the function $t \in\left(-\delta_{k}, \delta_{k}\right) \mapsto \mathcal{H}^{1}\left(\mathcal{M}_{t}^{k} \cap \bar{\omega}\right)$ is upper semicontinuous (see e.g. [4, Proposition 1.62]). In particular,

$$
\limsup _{t \rightarrow 0} \mathcal{H}^{1}\left(\mathcal{M}_{t}^{k} \cap \bar{\omega}\right) \leqslant \mathcal{H}^{1}\left(\mathcal{M}^{k} \cap \bar{\omega}\right) .
$$

Next we consider for each $k \in \mathbb{N}$, some $\ell_{k}>0$ and $v_{k} \in C^{2}\left(\ell_{k} I \times \gamma I ; \mathbb{R}^{3}\right)$ satisfying $\nabla v_{k}(y)=\left(0, \bar{b}_{0}\left(y_{1}\right)\right)$ nearby $\left\{\left|y_{1}\right|=\ell_{k} / 2\right\}$, and (4.27).

Let $h_{n} \rightarrow 0$ be an arbitrary sequence. Here again we can choose $\varepsilon_{n}:=h_{n} / \gamma$. For each $k \in \mathbb{N}$ and $n \in \mathbb{N}$ such that $\varepsilon_{n} \ell_{k}<\delta_{k}$, we define for $x \in \Omega$,

$$
u_{n, k}(x):= \begin{cases}\varepsilon_{n} v_{k}\left(\frac{d_{k}\left(x^{\prime}\right)}{\varepsilon_{n}}, \gamma x_{3}\right) & \text { if }\left|d_{k}\left(x^{\prime}\right)\right|<\frac{\ell_{k} \varepsilon_{n}}{2}, \\ \varepsilon_{n} v_{k}\left(\frac{\ell_{k}}{2}, \gamma x_{3}\right) & \text { if } d_{k}\left(x^{\prime}\right) \geqslant \frac{\ell_{k} \varepsilon_{n}}{2}, \\ \varepsilon_{n} v_{k}\left(-\frac{\ell_{k}}{2}, \gamma x_{3}\right) & \text { if } d_{k}\left(x^{\prime}\right) \leqslant-\frac{\ell_{k} \varepsilon_{n}}{2} .\end{cases}
$$

Then $u_{n, k} \in H^{2}\left(\Omega ; \mathbb{R}^{3}\right)$, and

$\nabla_{h_{n}} u_{n}(x)= \begin{cases}\left(\partial_{1} v_{k}\left(\frac{d_{k}\left(x^{\prime}\right)}{\varepsilon_{n}}, \gamma x_{3}\right) \otimes \nabla d_{k}\left(x^{\prime}\right), \partial_{2} v_{k}\left(\frac{d_{k}\left(x^{\prime}\right)}{\varepsilon_{n}}, \gamma x_{3}\right)\right) & \text { if }\left|d_{k}\left(x^{\prime}\right)\right|<\frac{\ell_{k} \varepsilon_{n}}{2}, \\ \left(0, b_{k}(x)\right) & \text { otherwise. }\end{cases}$

From the boundedness of $v_{k}$ and $\nabla v_{k}$ together with the smoothness of $d_{k}$ in $U_{k}$, we infer that $u_{n, k} \rightarrow 0$ in $W^{1, p}\left(\Omega ; \mathbb{R}^{3}\right)$ and $\frac{1}{h_{n}} \partial_{3} u_{n, k} \rightarrow b_{k}$ in $L^{p}\left(\Omega ; \mathbb{R}^{3}\right)$ as $n \rightarrow \infty$. Now it remains to estimate $F_{\varepsilon_{n}}^{h_{n}}\left(u_{n, k}\right)$. First of all, (4.39) yields $F_{\varepsilon_{n}}^{h_{n}}\left(u_{n, k}, \Omega \backslash\left\{\left|d_{k}\left(x^{\prime}\right)\right|<\ell_{k} \varepsilon_{n} / 2\right\}\right)=0$. Using the fact that $\left|\nabla d_{k}\right|=1 \mathcal{L}^{2}$-a.e. in $\mathbb{R}^{2}$, we infer from $\left(H_{5}\right)$ that for $x \in \Omega \cap\left\{\left|d_{k}\left(x^{\prime}\right)\right|<\ell_{k} \varepsilon_{n} / 2\right\}$,

$$
\begin{aligned}
& W\left(\nabla_{h_{n}} u_{n, k}(x)\right)=V\left(\left|\partial_{1} v_{k}\left(d_{k}\left(x^{\prime}\right) / \varepsilon_{n}, \gamma x_{3}\right)\right|, \partial_{2} v_{k}\left(d_{k}\left(x^{\prime}\right) / \varepsilon_{n}, \gamma x_{3}\right)\right) \\
& \quad=\mathcal{W}\left(\nabla v_{k}\left(d_{k}\left(x^{\prime}\right) / \varepsilon_{n}, \gamma x_{3}\right)\right) .
\end{aligned}
$$

Next we compute for $x \in \Omega \cap\left\{\left|d_{k}\left(x^{\prime}\right)\right|<\ell_{k} \varepsilon_{n} / 2\right\}$,

$$
\begin{aligned}
\left|\nabla_{h_{n}}^{2} u_{n, k}(x)\right|^{2}= & \frac{1}{\varepsilon_{n}^{2}}\left|\nabla^{2} v_{k}\left(d_{k}\left(x^{\prime}\right) / \varepsilon_{n}, \gamma x_{3}\right)\right|^{2}+\left|\partial_{1} v_{k}\left(d_{k}\left(x^{\prime}\right) / \varepsilon_{n}, \gamma x_{3}\right)\right|^{2}\left|\nabla^{2} d_{k}\left(x^{\prime}\right)\right|^{2} \\
& +\frac{2}{\varepsilon_{n}}\left(\partial_{1} v_{k}\left(d_{k}\left(x^{\prime}\right) / \varepsilon_{n}, \gamma x_{3}\right) \cdot \partial_{1}^{2} v_{k}\left(d_{k}\left(x^{\prime}\right) / \varepsilon_{n}, \gamma x_{3}\right)\right) \\
& \times\left(\nabla^{2} d_{k}\left(x^{\prime}\right) \cdot\left(\nabla d_{k}\left(x^{\prime}\right) \otimes \nabla d_{k}\left(x^{\prime}\right)\right)\right),
\end{aligned}
$$

which yields

$$
\left|\nabla_{h_{n}}^{2} u_{n, k}(x)\right|^{2} \leqslant \frac{1+\varepsilon_{n}}{\varepsilon_{n}^{2}}\left|\nabla^{2} v_{k}\left(d_{k}\left(x^{\prime}\right) / \varepsilon_{n}, \gamma x_{3}\right)\right|^{2}+\frac{C_{k}}{\varepsilon_{n}}\left|\partial_{1} v_{k}\left(d_{k}\left(x^{\prime}\right) / \varepsilon_{n}, \gamma x_{3}\right)\right|^{2}
$$


for $x \in \Omega \cap\left\{\left|d_{k}\left(x^{\prime}\right)\right|<\ell_{k} \varepsilon_{n} / 2\right\}$ and some constant $C_{k}$ independent of $n$. Therefore,

$$
F_{\varepsilon_{n}}^{h_{n}}\left(u_{n, k}\right)=F_{\varepsilon_{n}}^{h_{n}}\left(u_{n, k}, \Omega \cap\left\{\left|d_{k}\left(x^{\prime}\right)\right|<\ell_{k} \varepsilon_{n} / 2\right\}\right) \leqslant I_{n}^{k}+I I_{n}^{k},
$$

with

$I_{n}^{k}:=\frac{1}{\varepsilon_{n}} \int_{\Omega \cap\left\{\left|d_{k}\right|<\ell_{k} \varepsilon_{n} / 2\right\}} \mathcal{W}\left(\nabla v_{k}\left(d_{k}\left(x^{\prime}\right) / \varepsilon_{n}, \gamma x_{3}\right)\right)+\left|\nabla^{2} v_{k}\left(d_{k}\left(x^{\prime}\right) / \varepsilon_{n}, \gamma x_{3}\right)\right|^{2} d x$, and

$I I_{n}^{k}:=\int_{\Omega \cap\left\{\left|d_{k}\right|<\ell_{k} \varepsilon_{n} / 2\right\}}\left|\nabla^{2} v_{k}\left(d_{k}\left(x^{\prime}\right) / \varepsilon_{n}, \gamma x_{3}\right)\right|^{2}+C_{k}\left|\partial_{1} v_{k}\left(d_{k}\left(x^{\prime}\right) / \varepsilon_{n}, \gamma x_{3}\right)\right|^{2} d x$.

Using Fubini's theorem, the Coarea Formula, the fact that $\left|\nabla d_{k}\right|=1$, and changing variables we estimate

$$
\begin{aligned}
I_{n}^{k} & =\frac{1}{\varepsilon_{n}} \int_{I}\left(\int_{\omega \cap\left\{\left|d_{k}\right|<\ell_{k} \varepsilon_{n} / 2\right\}} \mathcal{W}\left(\nabla v_{k}\left(d_{k}\left(x^{\prime}\right) / \varepsilon_{n}, \gamma x_{3}\right)\right)+\left|\nabla^{2} v_{k}\left(d_{k}\left(x^{\prime}\right) / \varepsilon_{n}, \gamma x_{3}\right)\right|^{2} d x^{\prime}\right) d x_{3} \\
& =\frac{1}{\varepsilon_{n}} \int_{I}\left(\int_{\ell_{k} \varepsilon_{n} I}\left(\mathcal{W}\left(\nabla v_{k}\left(t / \varepsilon_{n}, \gamma x_{3}\right)\right)+\left|\nabla^{2} v_{k}\left(t / \varepsilon_{n}, \gamma x_{3}\right)\right|^{2}\right) \mathcal{H}^{1}\left(\mathcal{M}_{t}^{k} \cap \omega\right) d t\right) d x_{3} \\
& =\frac{1}{\varepsilon_{n}} \int_{\ell_{k} \varepsilon_{n} I \times I}\left(\mathcal{W}\left(\nabla v_{k}\left(t / \varepsilon_{n}, \gamma x_{3}\right)\right)+\left|\nabla^{2} v_{k}\left(t / \varepsilon_{n}, \gamma x_{3}\right)\right|^{2}\right) \mathcal{H}^{1}\left(\mathcal{M}_{t}^{k} \cap \omega\right) d t d x_{3} \\
& \leqslant \frac{1}{\gamma} \int_{\ell_{k} I \times \gamma I}\left(\mathcal{W}\left(\nabla v_{k}(y)\right)+\left|\nabla^{2} v_{k}(y)\right|^{2}\right) \mathcal{H}^{1}\left(\mathcal{M}_{\varepsilon_{n} y_{1}}^{k} \cap \bar{\omega}\right) d y .
\end{aligned}
$$

Then Fatou's lemma, (4.38), and (4.27) yield

$$
\limsup _{n \rightarrow \infty} I_{n}^{k} \leqslant\left(K_{\gamma}+2^{-k}\right) \mathcal{H}^{1}\left(\mathcal{M}^{k} \cap \bar{\omega}\right) \text {. }
$$

Arguing in the same way we infer that

$$
\lim _{n \rightarrow \infty} I I_{n}^{k}=\lim _{n \rightarrow+\infty} \frac{\varepsilon_{n}}{\gamma} \int_{\ell_{k} I \times \gamma I}\left(\left|\nabla^{2} v_{k}(y)\right|^{2}+C_{k}\left|\partial_{1} v_{k}(y)\right|^{2}\right) \mathcal{H}^{1}\left(\mathcal{M}_{\varepsilon_{n} y_{1}} \cap \bar{\omega}\right) d y=0
$$

Gathering (4.40)-(4.42), we derive

$$
\limsup _{k \rightarrow \infty} \limsup _{n \rightarrow \infty} F_{\varepsilon_{n}}^{h_{n}}\left(u_{n, k}\right) \leqslant K_{\gamma} \operatorname{Per}_{\omega}(E)
$$

Since $\lim _{k} \lim _{n}\left\|u_{n, k}\right\|_{W^{1, p}(\Omega)}=0$, and $\lim _{k} \lim _{n}\left\|\frac{1}{h_{n}} \partial_{3} u_{n, k}-b\right\|_{L^{p}(\Omega)}=$ $\lim _{k}\left\|b_{k}-b\right\|_{L^{p}(\Omega)}=0$, the conclusion follows for a suitable diagonal sequence $u_{n}:=u_{n, k_{n}}$ as in Step 1 .

\section{5. $\Gamma$-convergence in the subcritical regime}

This section is devoted to the proof of Theorem 1.3. The $\Gamma$-liminf inequality is obtained through a slicing argument, and by establishing a lower asymptotic inequality for a reduced 2D functional (see Proposition 5.1) much in the spirit of Sect. 4.1. The $\Gamma$-lim inf and $\Gamma$-lim sup inequalities are stated in Theorem 5.3 and Theorem 5.7 respectively, and Corollary 5.6 shows that lower and upper inequalities agree. 


\subsection{The $\Gamma$ - lim inf inequality}

For a bounded open set $A \subset \mathbb{R}^{2}$ and $\varepsilon>0$, we introduce the localized functional $F_{\varepsilon}^{0}(\cdot, \cdot, A)$ defined for a pair $(u, b) \in H^{2}\left(A ; \mathbb{R}^{3}\right) \times H^{1}\left(A ; \mathbb{R}^{3}\right)$ by

$$
F_{\varepsilon}^{0}(u, b, A):=\int_{A} \frac{1}{\varepsilon} W\left(\nabla^{\prime} u, b\right)+\varepsilon\left(\left|\left(\nabla^{\prime}\right)^{2} u\right|^{2}+2\left|\nabla^{\prime} b\right|^{2}\right) d x^{\prime} .
$$

Then we consider the constant

$$
\begin{gathered}
K_{0}^{\star}:=\inf \left\{\liminf _{n \rightarrow \infty} F_{\varepsilon_{n}}^{0}\left(u_{n}, b_{n}, Q^{\prime}\right): \varepsilon_{n} \rightarrow 0^{+},\left\{\left(u_{n}, b_{n}\right)\right\} \subset H^{2}\left(Q^{\prime} ; \mathbb{R}^{3}\right) \times H^{1}\left(Q^{\prime} ; \mathbb{R}^{3}\right),\right. \\
\left.\left(u_{n}, b_{n}\right) \rightarrow\left(u_{0}, b_{0}\right) \text { in }\left[L^{1}\left(Q^{\prime} ; \mathbb{R}^{3}\right)\right]^{2}\right\} .
\end{gathered}
$$

Here again the constant $K_{0}^{\star}$ is finite, as one may check by considering an admissible sequence $\left\{\left(u_{n}, b_{n}\right)\right\}$ made of suitable (standard) regularizations of $u_{0}$ and $b_{0}$. As in the previous section, we first provide a lower bound in terms of $K_{0}^{\star}$ for the lower $\Gamma$-limit of the family $\left\{F_{\varepsilon}^{0}\right\}$ in case of an elementary jump set.

Proposition 5.1. Assume that assumptions $\left(H_{1}\right),\left(H_{2}\right)$ and (1.5) hold. Let $\varepsilon_{n} \rightarrow 0^{+}$be an arbitrary sequence. Let $\rho>0$ and $\alpha \in \mathbb{R}$, let $J \subset \mathbb{R}$ be a bounded open interval, and consider the cylinder $U^{\prime}:=(\alpha-\rho, \alpha+\rho) \times J$. Let $(u, b) \in W^{1, \infty}\left(U^{\prime} ; \mathbb{R}^{3}\right) \times L^{\infty}\left(U^{\prime} ; \mathbb{R}^{3}\right)$ satisfying (4.2). Then for any sequence $\left\{\left(u_{n}, b_{n}\right)\right\} \subset H^{2}\left(U^{\prime} ; \mathbb{R}^{3}\right) \times H^{1}\left(U^{\prime} ; \mathbb{R}^{3}\right)$ such that $\left(u_{n}, b_{n}\right) \rightarrow(u, b)$ in $\left[L^{1}\left(U^{\prime} ; \mathbb{R}^{3}\right)\right]^{2}$, we have

$$
\liminf _{n \rightarrow \infty} F_{\varepsilon_{n}}^{0}\left(u_{n}, b_{n}, U^{\prime}\right) \geqslant K_{0}^{\star} \mathcal{H}^{1}(J) .
$$

Proof. Here the proof closely follows the one of Proposition 4.1. The arguments are essentially the same with the obvious modifications once we consider

$$
\begin{gathered}
\mathcal{E}_{0}(J, \rho):=\inf \left\{\liminf _{n \rightarrow \infty} F_{\varepsilon_{n}}^{0}\left(u_{n}, b_{n}, J_{\rho}^{\prime}\right): \varepsilon_{n} \rightarrow 0^{+},\left\{\left(u_{n}, b_{n}\right)\right\} \subset H^{2}\left(J_{\rho}^{\prime} ; \mathbb{R}^{3}\right) \times H^{1}\left(J_{\rho}^{\prime} ; \mathbb{R}^{3}\right),\right. \\
\left.\left(u_{n}, b_{n}\right) \rightarrow\left(u_{0}, b_{0}\right) \text { in }\left[L^{1}\left(J_{\rho}^{\prime} ; \mathbb{R}^{3}\right)\right]^{2}\right\}
\end{gathered}
$$

in place of $\mathcal{E}_{\gamma}(J, \rho)$ with $J \subset \mathbb{R}$ a bounded open set, $\rho>0$, and $J_{\rho}^{\prime}:=\rho I \times J$. Then one proves the analogue of Lemma 4.2 , in particular that $\mathcal{E}_{0}(J, \rho)=$ $K_{0}^{\star} \mathcal{H}^{1}(J)$. We omit any further details.

Remark 5.2. As in Corollary 4.4, the energy of optimal sequences for $\mathcal{E}_{0}(J, \rho)$ is concentrated near the limiting interface, i.e., given $0<\delta<\rho$, for any sequences $\varepsilon_{n} \rightarrow 0^{+}$and $\left\{\left(u_{n}, b_{n}\right)\right\} \subset H^{2}\left(J_{\rho}^{\prime} ; \mathbb{R}^{3}\right) \times H^{1}\left(J_{\rho}^{\prime} ; \mathbb{R}^{3}\right)$ such that $\left(u_{n}, b_{n}\right) \rightarrow\left(u_{0}, b_{0}\right)$ in $\left[L^{1}\left(J_{\rho}^{\prime} ; \mathbb{R}^{3}\right)\right]^{2}$ and $\lim _{n} F_{\varepsilon_{n}}^{0}\left(u_{n}, b_{n}, J_{\rho}^{\prime}\right)=\mathcal{E}_{0}(J, \rho)$, we have $\lim _{n} F_{\varepsilon_{n}}^{0}\left(u_{n}, b_{n}, J_{\rho}^{\prime} \backslash J_{\delta}^{\prime}\right)=0$.

We now prove the lower inequality for the $\Gamma$ - liminf of $\left\{F_{\varepsilon}^{h}\right\}$ essentially as in Theorem 4.6 together with a slicing argument involving the functionals $\left\{F_{\varepsilon}^{0}\right\}$.

Theorem 5.3. Assume that assumptions $\left(H_{1}\right)-\left(H_{2}\right),\left(H_{5}\right)$ and (1.5) hold. Let $h_{n} \rightarrow 0^{+}$and $\varepsilon_{n} \rightarrow 0^{+}$be arbitrary sequences such that $h_{n} / \varepsilon_{n} \rightarrow 0$. Then, for any $(u, b) \in \mathscr{C}$ and any sequence $\left\{u_{n}\right\} \subset H^{2}\left(\Omega ; \mathbb{R}^{3}\right)$ such that $\left(u_{n}, \frac{1}{h_{n}} \partial_{3} u_{n}\right) \rightarrow$ $(u, b)$ in $\left[L^{1}\left(\Omega ; \mathbb{R}^{3}\right)\right]^{2}$, we have

$$
\liminf _{n \rightarrow \infty} F_{\varepsilon_{n}}^{h_{n}}\left(u_{n}\right) \geqslant K_{0}^{\star} \operatorname{Per}_{\omega}(E),
$$


where $\left(\nabla^{\prime} u, b\right)(x)=\left(1-\chi_{E}\left(x^{\prime}\right)\right) A+\chi_{E}\left(x^{\prime}\right) B$.

Proof. Step 1. First we may assume that $\liminf _{n} F_{\varepsilon_{n}}^{h_{n}}\left(u_{n}\right)=\lim _{n} F_{\varepsilon_{n}}^{h_{n}}\left(u_{n}\right)<$ $\infty$. We set $b_{n}:=\frac{1}{h_{n}} \partial_{3} u_{n} \in H^{1}\left(\Omega ; \mathbb{R}^{3}\right)$. It is well known that for $\mathcal{L}^{1}$-a.e. $x_{3} \in I$ the slices $u_{n}^{x_{3}}\left(x^{\prime}\right):=u_{n}\left(x^{\prime}, x_{3}\right)$ and $b_{n}^{x_{3}}\left(x^{\prime}\right):=b_{n}\left(x^{\prime}, x_{3}\right)$ belong to $H^{2}\left(\omega ; \mathbb{R}^{3}\right)$ and $H^{1}\left(\omega ; \mathbb{R}^{3}\right)$ respectively, and horizontal weak derivatives coincide $\mathcal{L}^{3}$-a.e. in $\Omega$ (see e.g. [4, p. 204]). Moreover, up to a subsequence, $\left(u_{n}^{x_{3}}, b_{n}^{x_{3}}\right) \rightarrow(u, b)$ in $\left[L^{1}\left(\omega ; \mathbb{R}^{3}\right)\right]^{2}$ for $\mathcal{L}^{1}$-a.e. $x_{3} \in I$. Hence, using Fubini's theorem we can estimate

$$
\begin{aligned}
F_{\varepsilon_{n}}^{h_{n}}\left(u_{n}\right) & =\int_{I}\left(\int_{\omega \times\left\{x_{3}\right\}} \frac{1}{\varepsilon_{n}} W\left(\nabla_{h_{n}} u_{n}\right)+\varepsilon_{n}\left|\nabla_{h_{n}}^{2} u_{n}\right|^{2} d \mathcal{H}^{2}\right) d x_{3} \\
& \geqslant \int_{I} F_{\varepsilon_{n}}^{0}\left(u_{n}^{x_{3}}, b_{n}^{x_{3}}, \omega\right) d x_{3},
\end{aligned}
$$

and then infer from Fatou's lemma that

$$
\lim _{n \rightarrow \infty} F_{\varepsilon_{n}}^{h_{n}}\left(u_{n}\right) \geqslant \int \liminf _{I} F_{\varepsilon_{n}}^{0}\left(u_{n}^{x_{3}}, b_{n}^{x_{3}}, \omega\right) d x_{3} .
$$

Now it remains to prove that for $\mathcal{L}^{1}$-a.e. $x_{3} \in I$,

$$
\liminf _{n \rightarrow \infty} F_{\varepsilon_{n}}^{0}\left(u_{n}^{x_{3}}, b_{n}^{x_{3}}, \omega\right) \geqslant K_{0}^{\star} \operatorname{Per}_{\omega}(E) .
$$

The next steps are devoted to the proof of (5.3).

Step 2. First assume that $A^{\prime} \neq B^{\prime}$. We obtain estimate (5.3) by applying Proposition 5.1 together with the covering argument used in the proof of Theorem 4.6, Step 1. Further details are left to the reader.

Step 3. We now consider the case $A^{\prime}=B^{\prime}(=0$ by (1.5)), and we may assume that $u \equiv 0$. Then consider an arbitrary sequence $\left\{\left(u_{n}, b_{n}\right)\right\} \subset$ $H^{2}\left(\omega ; \mathbb{R}^{3}\right) \times H^{1}\left(\omega ; \mathbb{R}^{3}\right)$ satisfying $\left(u_{n}, b_{n}\right) \rightarrow(0, b)$ in $\left[L^{1}\left(\omega ; \mathbb{R}^{3}\right)\right]^{2}$. We may also assume that $\liminf { }_{n} F_{\varepsilon_{n}}^{0}\left(u_{n}, b_{n}\right)=\lim _{n} F_{\varepsilon_{n}}^{0}\left(u_{n}, b_{n}\right)<\infty$. By Theorem 2.1 we have $b\left(x^{\prime}\right)=\left(1-\chi_{E}\left(x^{\prime}\right)\right) A_{3}+\chi_{E}\left(x^{\prime}\right) B_{3}$ for a set $E \subset \omega$ of finite perimeter in $\omega$. We prove the announced result following the blow-up argument in the proof of Theorem 4.6, Step 3. We introduce the finite nonnegative Radon measure $\mu_{n}$ on $\omega$ given by

$$
\mu_{n}:=\left(\frac{1}{\varepsilon_{n}} W\left(\nabla^{\prime} u_{n}, b_{n}\right)+\varepsilon_{n}\left(\left|\left(\nabla^{\prime}\right)^{2} u_{n}\right|^{2}+2\left|\nabla^{\prime} b_{n}\right|^{2}\right)\right) \mathscr{L}^{2}\lfloor\omega .
$$

Then $\mu_{n}(\omega)=F_{\varepsilon_{n}}^{0}\left(u_{n}, b_{n}\right), \sup _{n} \mu_{n}(\omega)<\infty$, and there is a subsequence (not relabeled) such that $\mu_{n} \rightarrow \mu$ weakly* in the sense of measures for some finite nonnegative Radon measure $\mu$ on $\omega$. By lower semicontinuity we have $\mu(\omega) \leqslant \lim _{n} F_{\varepsilon_{n}}^{0}\left(u_{n}, b_{n}\right)$, and we have to prove that $\mu(\omega) \geqslant K_{0}^{\star} \mathcal{H}^{1}\left(\partial^{*} E \cap \omega\right)$. This estimate can be achieved as in the proof of Theorem 4.6, Step 3, with minor modifications.

Remark 5.4. Let $\varepsilon_{n} \rightarrow 0^{+}$be an arbitrary sequence. By the arguments above, for any $(u, b) \in \mathscr{C}$ and any sequence $\left\{\left(u_{n}, b_{n}\right)\right\} \subset H^{2}\left(\omega ; \mathbb{R}^{3}\right) \times H^{1}\left(\omega ; \mathbb{R}^{3}\right)$ satisfying $\left(u_{n}, b_{n}\right) \rightarrow(u, b)$ in $\left[L^{1}\left(\omega ; \mathbb{R}^{3}\right)\right]^{2}$, we have $\liminf _{n} F_{\varepsilon_{n}}^{0}\left(u_{n}, b_{n}, \omega\right) \geqslant$ $K_{0}^{\star} \operatorname{Per}_{\omega}(E)$ where $\left(\nabla^{\prime} u, b\right)=\left(1-\chi_{E}\right) A+\chi_{E} B$. 


\subsection{Lower bound on $K_{0}^{\star}$}

As in Proposition 4.8, we now prove that sequences realizing $K_{0}^{\star}$ can be prescribed near the two sides $\left\{x_{1}= \pm \frac{1}{2}\right\}$, and chosen to be independent of the $x_{2}$-variable.

Proposition 5.5. Assume that $\left(H_{1}\right)-\left(H_{4}\right)$ and (1.5) hold. Then there exist sequences $\varepsilon_{n} \rightarrow 0^{+},\left\{c_{n}\right\} \subset \mathbb{R}^{3}$, and $\left\{\left(g_{n}, d_{n}\right)\right\} \subset C^{2}\left(Q^{\prime} ; \mathbb{R}^{3}\right) \times C^{1}\left(Q^{\prime} ; \mathbb{R}^{3}\right)$ such that $\left(g_{n}, d_{n}\right)$ is independent of $x_{2}\left(\right.$ i.e., $g_{n}\left(x^{\prime}\right)=: \bar{g}_{n}\left(x_{1}\right)$ and $d_{n}\left(x^{\prime}\right)=$ : $\left.\bar{d}_{n}\left(x_{1}\right)\right), c_{n} \rightarrow 0, g_{n} \rightarrow u_{0}$ in $W^{1, p}\left(Q^{\prime} ; \mathbb{R}^{3}\right), d_{n} \rightarrow b_{0}$ in $L^{p}\left(Q^{\prime} ; \mathbb{R}^{3}\right)$,

$$
\begin{aligned}
& \left(g_{n}, d_{n}\right)=\left(u_{0}, b_{0}\right) \text { in } Q^{\prime} \cap\left\{x_{1}>1 / 4\right\}, \\
& \left(g_{n}, d_{n}\right)=\left(u_{0}+c_{n}, b_{0}\right) \text { in } Q^{\prime} \cap\left\{x_{1}<-1 / 4\right\},
\end{aligned}
$$

and $\lim _{n} F_{\varepsilon_{n}}^{0}\left(g_{n}, d_{n}, Q^{\prime}\right)=K_{0}^{\star}$.

Proof. Step 1. Consider sequences $\varepsilon_{n} \rightarrow 0^{+}$and $\left\{\left(u_{n}, b_{n}\right)\right\} \subset H^{2}\left(Q^{\prime} ; \mathbb{R}^{3}\right) \times$ $H^{1}\left(Q^{\prime} ; \mathbb{R}^{3}\right)$ such that $\left(u_{n}, b_{n}\right) \rightarrow\left(u_{0}, b_{0}\right)$ in $\left[L^{1}\left(Q^{\prime} ; \mathbb{R}^{3}\right)\right]^{2}$, and $\lim _{n} F_{\varepsilon_{n}}^{0}$ $\left(u_{n}, b_{n}, Q^{\prime}\right)=K_{0}^{\star}$. Arguing as in the proof of Proposition 4.9, we may assume that $\left(u_{n}, b_{n}\right) \in C^{2}\left(Q^{\prime} ; \mathbb{R}^{3}\right) \times C^{1}\left(Q^{\prime} ; \mathbb{R}^{3}\right)$, and that $\left(u_{n}, b_{n}\right)$ is independent of $x_{2}$, i.e., $\left(u_{n}, b_{n}\right)(x)=:\left(\bar{u}_{n}\left(x_{1}\right), \bar{b}_{n}\left(x_{1}\right)\right)$. Moreover, the arguments used in the proof of Theorem 1.1 (with minor modifications) yield $u_{n} \rightarrow u_{0}$ in $W^{1, p}\left(Q^{\prime} ; \mathbb{R}^{3}\right)$, and $b_{n} \rightarrow b_{0}$ in $L^{p}\left(Q^{\prime} ; \mathbb{R}^{3}\right)$.

Step 2. Here again we consider a partition of $\left(\frac{1}{12}, \frac{1}{6}\right)$ into $M_{n}:=\left[\frac{1}{\varepsilon_{n}}\right]$ intervals of length $\frac{1}{12 M_{n}}$. By Remark 5.2, the energy concentrates near the interface $\left\{x_{1}=0\right\}$, and we can find a suitable interval $I_{n}:=\left(\theta_{n}-\frac{1}{12 M_{n}}, \theta_{n}\right) \subset$ $\left(\frac{1}{12}, \frac{1}{6}\right)$ for which

$$
\begin{aligned}
& M_{n}\left(\int_{I_{n}}\left|\bar{u}_{n}-\bar{u}_{0}\right|^{p}+\left|\bar{b}_{n}-\bar{b}_{0}\right|^{p}+\left|\bar{u}_{n}^{\prime}-\bar{u}_{0}^{\prime}\right|^{p} d x_{1}+F_{\varepsilon_{n}}^{0}\left(u_{n}, b_{n}, I_{n} \times I\right)\right) \\
& \quad \leqslant \int_{\left(\frac{1}{12}, \frac{1}{6}\right)}\left|\bar{u}_{n}-\bar{u}_{0}\right|^{p}+\left|\bar{b}_{n}-\bar{b}_{0}\right|^{p}+\left|\bar{u}_{n}^{\prime}-\bar{u}_{0}^{\prime}\right|^{p} d x_{1}+F_{\varepsilon_{n}}^{0}\left(u_{n}, b_{n},\left(\frac{1}{12}, \frac{1}{6}\right) \times I\right)=: \alpha_{n} \rightarrow 0 .
\end{aligned}
$$

We select a level $t_{n} \in\left(\theta_{n}-\frac{1}{12 M_{n}}, \theta_{n}\right)$ satisfying

$$
\begin{aligned}
& \left|\bar{u}_{n}\left(t_{n}\right)-\bar{u}_{0}\left(t_{n}\right)\right|^{p}+\left|\bar{b}_{n}\left(t_{n}\right)-\bar{b}_{0}\left(t_{n}\right)\right|^{p}+\left|\bar{u}_{n}^{\prime}\left(t_{n}\right)-\bar{u}_{0}^{\prime}\left(t_{n}\right)\right|^{p} \\
& \quad+\frac{1}{\varepsilon_{n}} W\left(\bar{u}_{n}^{\prime}\left(t_{n}\right), 0, \bar{b}_{n}\left(t_{n}\right)\right)+\varepsilon_{n}\left|\bar{u}_{n}^{\prime \prime}\left(t_{n}\right)\right|^{2}+2 \varepsilon_{n}\left|\bar{b}_{n}^{\prime}\left(t_{n}\right)\right|^{2} \leqslant 12 \alpha_{n} .
\end{aligned}
$$

Let $\varphi_{n} \in C^{\infty}(\mathbb{R})$ be a cut-off function as in (4.10). For $x_{1} \in I_{n}$ we set

$$
v_{n}\left(x_{1}\right):=\left(1-\varphi_{n}\left(x_{1}\right)\right)\left(\bar{u}_{0}\left(x_{1}\right)+c_{n}^{+}\right)+\varphi_{n}\left(x_{1}\right) \bar{u}_{n}\left(x_{1}\right),
$$

with $c_{n}^{+}:=\bar{u}_{n}\left(t_{n}\right)-\bar{u}_{0}\left(t_{n}\right) \rightarrow 0$, and

$$
\zeta_{n}\left(x_{1}\right):=\left(1-\varphi_{n}\left(x_{1}\right)\right) \bar{b}_{0}\left(x_{1}\right)+\varphi_{n}\left(x_{1}\right) \bar{b}_{n}\left(x_{1}\right),
$$

We claim that

$$
\int_{I_{n}}\left|v_{n}-\bar{u}_{0}\right|^{p} d x_{1} \rightarrow 0
$$




$$
\begin{aligned}
& \frac{1}{\varepsilon_{n}} \int_{I_{n}}\left|\zeta_{n}-\bar{b}_{0}\right|^{p} d x_{1} \rightarrow 0, \\
& \frac{1}{\varepsilon_{n}} \int_{I_{n}}\left|v_{n}^{\prime}-\bar{u}_{0}^{\prime}\right|^{p} d x_{1} \rightarrow 0, \\
& \frac{1}{\varepsilon_{n}} \int_{I_{n}} W\left(v_{n}^{\prime}, 0, \zeta_{n}\right) d x_{1} \rightarrow 0, \\
& \varepsilon_{n} \int_{I_{n}}\left|v_{n}^{\prime \prime}\right|^{2}+2\left|\zeta_{n}^{\prime}\right|^{2} d x_{1} \rightarrow 0 .
\end{aligned}
$$

Estimates (5.6) and (5.7) come straightforward from (5.4). We apply Poincaré's inequality to obtain

$$
\int_{I_{n}}\left|\bar{u}_{n}-\bar{u}_{0}-c_{n}^{+}\right|^{p} d x_{1} \leqslant C\left(\frac{1}{M_{n}}\right)^{p} \int_{I_{n}}\left|\bar{u}_{n}^{\prime}-\bar{u}_{0}^{\prime}\right|^{p} d x_{1} \leqslant C \alpha_{n} \varepsilon_{n}^{p+1},
$$

and using (5.4), (4.10), and (5.11), we derive

$\frac{1}{\varepsilon_{n}} \int_{I_{n}}\left|v_{n}^{\prime}-\bar{u}_{0}^{\prime}\right|^{p} d x_{1} \leqslant \frac{C}{\varepsilon_{n}} \int_{I_{n}}\left(\frac{1}{\varepsilon_{n}^{p}}\left|\bar{u}_{n}-\bar{u}_{0}-c_{n}^{+}\right|^{p}+\left|\bar{u}_{n}^{\prime}-\bar{u}_{0}^{\prime}\right|^{p}\right) d x_{1} \leqslant C \alpha_{n} \rightarrow 0$,

so that (5.8) is proved. Now (5.9) follows from (5.7) and (5.8) exactly as (4.19). Finally we obtain (5.10) arguing as in the proof of Proposition 4.9 with minor modifications. We omit further details.

Step 3. We conclude as in the proof of Proposition 4.9, Step 4. We first define a sequence $\left(g_{n}^{+}, d_{n}^{+}\right)$by setting for $x^{\prime} \in Q^{\prime}$,

$$
\left(g_{n}^{+}, d_{n}^{+}\right)\left(x^{\prime}\right):= \begin{cases}\left(\bar{u}_{n}\left(x_{1}\right), \bar{b}_{n}\left(x_{1}\right)\right) & \text { for } x_{1}<\theta_{n}-\frac{1}{12 M_{n}} \\ \left(v_{n}\left(x_{1}\right), \zeta_{n}\left(x_{1}\right)\right) & \text { for } \theta_{n}-\frac{1}{12 M_{n}} \leqslant x_{1}<\theta_{n} \\ \left(\bar{u}_{0}\left(x_{1}\right)+c_{n}^{+}, \bar{b}_{0}\left(x_{1}\right)\right) & \text { for } \theta_{n} \leqslant x_{1} \leqslant \frac{1}{2}\end{cases}
$$

Then we repeat the procedure above to modify $g_{n}^{+}$in $\left(-\frac{1}{2}, 0\right) \times I$. Again, we omit further details.

Corollary 5.6. Assume that $\left(H_{1}\right)-\left(H_{4}\right)$ and (1.5) hold. Then $K_{0}^{\star} \geqslant K_{0}$.

Proof. Consider the sequences $\left\{\varepsilon_{n}\right\}$ and $\left\{\left(g_{n}, d_{n}\right)\right\}$ given by Proposition 5.5. We set $\ell_{n}:=\varepsilon_{n} / 2$, and for $t \in\left[-\ell_{n}, \ell_{n}\right], \phi_{n}(t):=\left(\phi_{1, n}, \phi_{2, n}\right)(t):=$ $\left(\bar{g}_{n}^{\prime}\left(t / \varepsilon_{n}\right), \bar{d}_{n}\left(t / \varepsilon_{n}\right)\right)$. Then straightforward computations yield $\phi_{n}=\left(\bar{u}_{0}^{\prime}, \bar{b}_{0}\right)$ nearby $\left\{|t|=\ell_{n}\right\}$, and

$$
\int_{-\ell_{n}}^{\ell_{n}} \mathcal{W}\left(\phi_{1, n}(t), \phi_{2, n}(t)\right)+\left|\phi_{1, n}^{\prime}(t)\right|^{2}+2\left|\phi_{2, n}^{\prime}(t)\right|^{2} d t=F_{\varepsilon_{n}}^{0}\left(g_{n}, d_{n}, Q^{\prime}\right) .
$$

By definition of $K_{0}$ and the construction of $\left\{\left(g_{n}, d_{n}\right)\right\}$ we have $K_{0} \leqslant$ $F_{\varepsilon_{n}}^{0}\left(g_{n}, d_{n}, Q^{\prime}\right) \rightarrow K_{0}^{\star}$ as $n \rightarrow \infty$, and the proof is complete.

\subsection{The $\Gamma$ - lim sup inequality}

We now complete the proof of Theorem 1.3 with the construction of recovery sequences. 
Theorem 5.7. Assume that $\left(H_{1}\right)-\left(H_{5}\right)$ and (1.5) hold. Let $\varepsilon_{n} \rightarrow 0^{+}$and $h_{n} \rightarrow 0^{+}$be arbitrary sequences such that $h_{n} / \varepsilon_{n} \rightarrow 0$. Then, for every $(u, b) \in \mathscr{C}$, there exists a sequence $\left\{u_{n}\right\} \subset H^{2}\left(\Omega ; \mathbb{R}^{3}\right)$ such that $u_{n} \rightarrow u$ in $W^{1, p}\left(\Omega ; \mathbb{R}^{3}\right), \frac{1}{h_{n}} \partial_{3} u_{n} \rightarrow b$ in $L^{p}\left(\Omega ; \mathbb{R}^{3}\right)$, and

$$
\lim _{n \rightarrow \infty} F_{\varepsilon_{n}}^{h_{n}}\left(u_{n}\right)=K_{0} \operatorname{Per}_{\omega}(E)
$$

where $\left(\nabla^{\prime} u, b\right)(x)=\left(1-\chi_{E}\left(x^{\prime}\right)\right) A+\chi_{E}\left(x^{\prime}\right) B$.

Proof. The proof parallels the one of Theorem 4.11, and we shall refer to it for the notation.

Step 1. We first assume that $A^{\prime} \neq B^{\prime}$, and that $\partial^{*} E \cap \omega$ is made by finitely many interfaces. We also assume that the pair $(u, b)$ is given by $(u, b)(x)=$ $(\bar{u}, \bar{b})\left(x_{1}\right)$ as in the proof of Theorem 4.11, Step 1 .

For $k \in \mathbb{N}$ arbitrary, we choose some $\ell_{k}>0$ and $\left(\phi_{1, k}, \phi_{2, k}\right): \mathbb{R} \rightarrow \mathbb{R}^{3 \times 2}$ of class $C^{1}$ such that $\left(\phi_{1, k}, \phi_{2, k}\right)=\left(\bar{u}_{0}^{\prime}, \bar{b}_{0}\right)$ in $\left\{|t| \geqslant \ell_{k} / 2\right\}$, and

$$
\int_{-\ell_{k} / 2}^{\ell_{k} / 2} \mathcal{W}\left(\phi_{1, k}(t), \phi_{2, k}(t)\right)+\left|\phi_{1, k}^{\prime}(t)\right|^{2}+2\left|\phi_{2, k}^{\prime}(t)\right|^{2} d t \leqslant K_{0}+2^{-k} .
$$

Without loss of generality, we may assume that $\phi_{2, k}^{\prime}$ is Lipschitz continuous. In the remaining of this step we shall drop the subscript $k$ for simplicity. For each $i=1, \ldots, m$ we fix some bounded open interval $J_{i}^{\prime} \subset \mathbb{R}$ satisfying (4.29), and we consider for $n$ large enough the coefficients $\left\{\alpha_{i \pm}^{n}\right\}$ as in (4.30), and such that (4.31) holds.

Let $h_{n} \rightarrow 0^{+}$and $\varepsilon_{n} \rightarrow 0^{+}$be arbitrary sequences such that $h_{n} / \varepsilon_{n} \rightarrow 0$, and define

$$
\Phi(t):=\int_{-\ell / 2}^{t} \phi_{1}(s) d s-c \quad \text { with } c:=\frac{1}{2} \int_{-\ell / 2}^{\ell / 2} \phi_{1}(s) d s .
$$

We set for $i=1, \ldots, m$ and $x \in\left(\alpha_{i-}^{n}, \alpha_{i+}^{n}\right) \times \mathbb{R} \times I$,

$$
w_{n}^{i}(x):=(-1)^{i+1} \Phi\left((-1)^{i+1} \frac{x_{1}-\alpha_{i}}{\varepsilon_{n}}\right)+\frac{h_{n}}{\varepsilon_{n}} x_{3} \phi_{2}\left((-1)^{i+1} \frac{x_{1}-\alpha_{i}}{\varepsilon_{n}}\right)+\left(1+(-1)^{i}\right) c .
$$

Then we have

$$
w_{n}^{i}\left(\alpha_{i-}^{n}, x_{2}, x_{3}\right)= \begin{cases}\Phi(-\ell / 2)+\frac{h_{n}}{\varepsilon_{n}} x_{3} \phi_{2}(-\ell / 2) & \text { if } i \text { is odd, } \\ \Phi(\ell / 2)+\frac{h_{n}}{\varepsilon_{n}} x_{3} \phi_{2}(\ell / 2) & \text { if } i \text { is even, }\end{cases}
$$

and

$$
w_{n}^{i}\left(\alpha_{i+}^{n}, x_{2}, x_{3}\right)= \begin{cases}\Phi(\ell / 2)+\frac{h_{n}}{\varepsilon_{n}} x_{3} \phi_{2}(\ell / 2) & \text { if } i \text { is odd } \\ \Phi(-\ell / 2)+\frac{h_{n}}{\varepsilon_{n}} x_{3} \phi_{2}(-\ell / 2)+4 c & \text { if } i \text { is even. }\end{cases}
$$


Setting $\beta_{i}^{n}$ and $\kappa_{i}$ as in (4.34), we define for $x \in \Omega$,

$$
:= \begin{cases}\bar{u}\left(x_{1}\right)+\varepsilon_{n} \Phi\left(\frac{-\ell}{2}\right)+h_{n} x_{3} \phi_{2}\left(\frac{-\ell}{2}\right) & \text { for } x_{1} \leqslant \alpha_{1-}^{n}, \\ \bar{u}\left(\alpha_{i-}^{n}\right)-\beta_{i-1}^{n}+\varepsilon_{n} w_{n}^{i}(x)+\varepsilon_{n} \kappa_{i-1} c & \text { for } \alpha_{i-}^{n}<x_{1}<\alpha_{i+}^{n}, \\ \bar{u}\left(x_{1}\right)-\beta_{i}^{n}+\varepsilon_{n} \Phi\left(\frac{(-1)^{i+1} \ell}{2}\right)+h_{n} x_{3} \phi_{2}\left(\frac{(-1)^{i+1} \ell}{2}\right)+\varepsilon_{n} \kappa_{i} c & \text { for } \alpha_{i+}^{n} \leqslant x_{1} \leqslant \alpha_{(i+1)-}^{n}, \\ \bar{u}\left(x_{1}\right)-\beta_{m}^{n}+\varepsilon_{n} \Phi\left(\frac{(-1)^{m+1} \ell}{2}\right)+h_{n} x_{3} \phi_{2}\left(\frac{(-1)^{m+1} \ell}{2}\right)+\varepsilon_{n} \kappa_{m} c & \text { for } x_{1} \geqslant \alpha_{m+}^{n} .\end{cases}
$$

In view of $(5.15)-(5.16)$, and since $\phi_{2}^{\prime}( \pm \ell / 2)=0$, we have $u_{n} \in H^{2}\left(\Omega ; \mathbb{R}^{3}\right)$. Moreover $u_{n}$ does not depend on $x_{2}$,

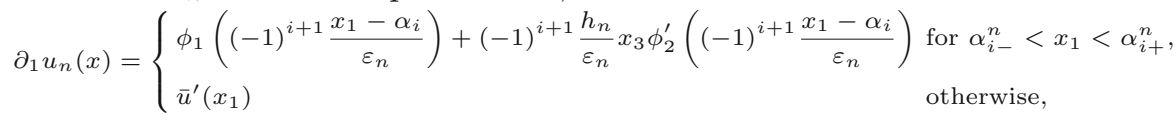

and

$$
\frac{1}{h_{n}} \partial_{3} u_{n}(x)= \begin{cases}\phi_{2}\left((-1)^{i+1} \frac{x_{1}-\alpha_{i}}{\varepsilon_{n}}\right) & \text { for } \alpha_{i-}^{n}<x_{1}<\alpha_{i+}^{n}, i=1, \ldots, m, \\ \bar{b}\left(x_{1}\right) & \text { otherwise. }\end{cases}
$$

Arguing as in the proof of Theorem 4.11, Step 1, we derive that $u_{n} \rightarrow u$ in $W^{1, p}\left(\Omega ; \mathbb{R}^{3}\right)$, and $\frac{1}{h_{n}} \partial_{3} u_{n} \rightarrow b$ in $L^{p}\left(\Omega ; \mathbb{R}^{3}\right)$. Then we estimate

$$
F_{\varepsilon_{n}}^{h_{n}}\left(u_{n}\right) \leqslant \sum_{i=1}^{m} F_{\varepsilon_{n}}^{h_{n}}\left(\varepsilon_{n} w_{n}^{i},\left(\alpha_{i-}^{n}, \alpha_{i+}^{n}\right) \times J_{i}^{\prime} \times I\right) .
$$

Changing variables and using Fubini's theorem, we obtain

$$
\begin{aligned}
F_{\varepsilon_{n}}^{h_{n}} & \left(\varepsilon_{n} w_{n}^{i},\left(\alpha_{i-}^{n}, \alpha_{i+}^{n}\right) \times J_{i}^{\prime} \times I\right) \\
= & \mathcal{H}^{1}\left(J_{i}^{\prime}\right) \int_{-\ell / 2}^{\ell / 2}\left(\int_{I} \mathcal{W}\left(\phi_{1}(t)+(-1)^{i+1} \frac{h_{n}}{\varepsilon_{n}} x_{3} \phi_{2}^{\prime}(t), \phi_{2}(t)\right) d x_{3}+\left|\phi_{1}^{\prime}(t)\right|^{2}\right. \\
& \left.+2\left|\phi_{2}^{\prime}(t)\right|^{2}\right) d t \\
& +\frac{\mathcal{H}^{1}\left(J_{i}^{\prime}\right) h_{n}^{2}}{12 \varepsilon_{n}^{2}} \int_{-\ell / 2}^{\ell / 2}\left|\phi_{2}^{\prime \prime}(t)\right|^{2} d t .
\end{aligned}
$$

Since $\mathcal{W}$ is continuous and $h_{n} / \varepsilon_{n} \rightarrow 0$, we infer that

$$
\begin{aligned}
& \lim _{n \rightarrow \infty} F_{\varepsilon_{n}}^{h_{n}}\left(\varepsilon_{n} w_{n}^{i},\left(\alpha_{i-}^{n}, \alpha_{i+}^{n}\right) \times J_{i}^{\prime} \times I\right)=\mathcal{H}^{1}\left(J_{i}^{\prime}\right) \int_{-\ell / 2}^{\ell / 2} \mathcal{W}\left(\phi_{1}(t), \phi_{2}(t)\right) \\
& \quad+\left|\phi_{1}^{\prime}(t)\right|^{2}+2\left|\phi_{2}^{\prime}(t)\right|^{2} d t,
\end{aligned}
$$

which leads to

$$
\limsup _{n \rightarrow \infty} F_{\varepsilon_{n}}^{h_{n}}\left(u_{n}\right) \leqslant K_{0} \operatorname{Per}_{\omega}(E)+C_{0} 2^{-k},
$$

for a constant $C_{0}$ which only depends on $m$ and $\operatorname{Per}_{\omega}(E)$. Then the conclusion follows for a suitable diagonal sequence as already pursued in the proof of Theorem 4.11.

Step 2. In the case where $A^{\prime} \neq B^{\prime}$ and $\partial^{*} E \cap \omega$ is made by infinitely many interfaces, the proof follows from the previous step through a diagonalization argument as in the proof of Theorem 4.11. 
Step 3. We now consider the case $A^{\prime}=B^{\prime}(=0)$, and we proceed as in the proof of Theorem 4.11 (we refer to it for the notation). We may assume that $u=0$, and $b(x)=\left(1-\chi_{E}\left(x^{\prime}\right)\right) A_{3}+\chi_{E}\left(x^{\prime}\right) B_{3}$ where $E \subset \omega$ has finite perimeter in $\omega$. We consider a sequence $\left\{E_{k}\right\}$ of smooth bounded subset of $\mathbb{R}^{2}$ such that $\chi_{E_{k}} \rightarrow \chi_{E}$ in $L^{1}(\omega)$, and $\lim _{k} \mathcal{H}^{1}\left(\partial E_{k} \cap \bar{\omega}\right)=\operatorname{Per}_{\omega}(E)$. We define $b_{k}:=\left(1-\chi_{E_{k}}\right) A_{3}+\chi_{E_{k}} B_{3}$, and the signed distance $d_{k}$ to $\mathcal{M}^{k}:=\partial E_{k}$ as in (4.37). Here again we shall drop the subscript $k$ for simplicity.

For $k \in \mathbb{N}$ arbitrary, we choose $\ell>0$ and $\left(\phi_{1}, \phi_{2}\right): \mathbb{R} \rightarrow \mathbb{R}^{3 \times 2}$ of class $C^{1}$ satisfying $\left(\phi_{1}, \phi_{2}\right)(t)=\left(0, \bar{b}_{0}(t)\right)$ nearby $\{|t|=\ell / 2\}$ and (5.13). We may also assume $\phi_{2}^{\prime}$ to be Lipschitz continuous. Defining $\Phi$ as in (5.14), we set for $x \in \Omega$,

$$
u_{n}(x)= \begin{cases}\varepsilon_{n} \Phi\left(\frac{d\left(x^{\prime}\right)}{\varepsilon_{n}}\right)+h_{n} x_{3} \phi_{2}\left(\frac{d\left(x^{\prime}\right)}{\varepsilon_{n}}\right) & \text { if }\left|d\left(x^{\prime}\right)\right|<\frac{\ell \varepsilon_{n}}{2}, \\ \varepsilon_{n} \Phi\left(\frac{\ell}{2}\right)+h_{n} x_{3} \phi_{2}\left(\frac{\ell}{2}\right) & \text { if } d\left(x^{\prime}\right) \geqslant \frac{\ell \varepsilon_{n}}{2}, \\ \varepsilon_{n} \Phi\left(-\frac{\ell}{2}\right)+h_{n} x_{3} \phi_{2}\left(-\frac{\ell}{2}\right) & \text { if } d\left(x^{\prime}\right) \leqslant-\frac{\ell \varepsilon_{n}}{2} .\end{cases}
$$

Then $u_{n} \in H^{2}\left(\Omega ; \mathbb{R}^{3}\right)$, and we compute

$$
\nabla^{\prime} u_{n}(x)=\left\{\begin{array}{c}
\left(\phi_{1}\left(\frac{d\left(x^{\prime}\right)}{\varepsilon_{n}}\right)+\frac{h_{n}}{\varepsilon_{n}} x_{3} \phi_{2}^{\prime}\left(\frac{d\left(x^{\prime}\right)}{\varepsilon_{n}}\right)\right) \otimes \nabla d\left(x^{\prime}\right) \\
\text { if }\left|d\left(x^{\prime}\right)\right|<\frac{\ell \varepsilon_{n}}{2}, \\
\text { otherwise, }
\end{array}\right.
$$

and

$$
\frac{1}{h_{n}} \partial_{3} u_{n}(x)= \begin{cases}\phi_{2}\left(\frac{d\left(x^{\prime}\right)}{\varepsilon_{n}}\right) & \text { if }\left|d\left(x^{\prime}\right)\right|<\frac{\ell \varepsilon_{n}}{2} \\ b(x) & \text { otherwise. }\end{cases}
$$

Since $|\nabla d|=1 \mathcal{L}^{2}$-a.e. in $\mathbb{R}^{2}$, in the set $\left\{|d|<\ell \varepsilon_{n} / 2\right\}$ we have

$$
\begin{aligned}
& \left|\nabla_{h_{n}}^{2} u_{n}\right|^{2}=\frac{1}{\varepsilon_{n}^{2}}\left|\phi_{1}^{\prime}\left(d / \varepsilon_{n}\right)+\frac{h_{n}}{\varepsilon_{n}} x_{3} \phi_{2}^{\prime \prime}\left(d / \varepsilon_{n}\right)\right|^{2}+2\left|\phi_{2}^{\prime}\left(d / \varepsilon_{n}\right)\right|^{2} \\
& \quad+\frac{1}{\varepsilon_{n}}\left(\phi_{1}^{\prime}\left(d / \varepsilon_{n}\right)+\frac{h_{n}}{\varepsilon_{n}} x_{3} \phi_{2}^{\prime \prime}\left(d / \varepsilon_{n}\right)\right) \cdot\left(\phi_{1}\left(d / \varepsilon_{n}\right)+\frac{h_{n}}{\varepsilon_{n}} x_{3} \phi_{2}^{\prime}\left(d / \varepsilon_{n}\right)\right)\left(\nabla^{2} d \cdot(\nabla d \otimes \nabla d)\right) \\
& \quad+\left|\phi_{1}\left(d / \varepsilon_{n}\right)+\frac{h_{n}}{\varepsilon_{n}} x_{3} \phi_{2}^{\prime}\left(d / \varepsilon_{n}\right)\right|^{2}\left|\nabla^{2} d\right|^{2} .
\end{aligned}
$$

As in the proof of Theorem 4.11, Step 3 , we derive that $u_{n} \rightarrow 0$ in $W^{1, p}\left(\Omega ; \mathbb{R}^{3}\right)$, and $\frac{1}{h_{n}} \partial_{3} u_{n} \rightarrow b_{k}$ in $L^{p}\left(\Omega ; \mathbb{R}^{3}\right)$. Then, using the fact that $|\nabla d|=1$ and assumption $\left(H_{5}\right)$, we estimate

$$
F_{\varepsilon_{n}}^{h_{n}}\left(u_{n}\right)=F_{\varepsilon_{n}}^{h_{n}}\left(u_{n}, \Omega \cap\left\{\left|d\left(x^{\prime}\right)\right|<\ell \varepsilon_{n} / 2\right\}\right) \leqslant I_{n}+C\left(\varepsilon_{n}+h_{n}^{2} / \varepsilon_{n}^{2}\right),
$$

with

$$
\begin{aligned}
I_{n}:= & \frac{1}{\varepsilon_{n}} \int_{\Omega \cap\left\{|d|<\ell \varepsilon_{n} / 2\right\}} \mathcal{W}\left(\phi_{1}\left(d\left(x^{\prime}\right) / \varepsilon_{n}\right)+\frac{h_{n}}{\varepsilon_{n}} x_{3} \phi_{2}^{\prime}\left(d\left(x^{\prime}\right) / \varepsilon_{n}\right), \phi_{2}\left(d\left(x^{\prime}\right) / \varepsilon_{n}\right)\right) d x \\
& +\frac{1}{\varepsilon_{n}} \int_{\Omega \cap\left\{|d|<\ell \varepsilon_{n} / 2\right\}}\left|\phi_{1}^{\prime}\left(d\left(x^{\prime}\right) / \varepsilon_{n}\right)\right|^{2}+2\left|\phi_{2}^{\prime}\left(d\left(x^{\prime}\right) / \varepsilon_{n}\right)\right|^{2} d x,
\end{aligned}
$$


and a constant $C$ independent of $n$. Using the Coarea Formula, we derive as in the proof of Theorem 4.11, Step 3, that

$$
I_{n}=\int_{-\ell / 2}^{\ell / 2}\left(\int_{I} \mathcal{W}\left(\phi_{1}(t)+\frac{h_{n}}{\varepsilon_{n}} x_{3} \phi_{2}^{\prime}(t), \phi_{2}(t)\right) d x_{3}+\left|\phi_{1}^{\prime}(t)\right|^{2}+2\left|\phi_{2}^{\prime}(t)\right|^{2}\right) \mathcal{H}^{1}\left(\mathcal{M}_{\varepsilon_{n} t}^{k} \cap \omega\right) d t
$$

Since $\mathcal{W}$ is continuous and $h_{n} / \varepsilon_{n} \rightarrow 0$, we infer from Fatou's lemma, (4.38), (5.13) and (5.17) that

$$
\limsup _{n \rightarrow \infty} F_{\varepsilon_{n}}^{h_{n}}\left(u_{n}\right) \leqslant\left(K_{0}+2^{-k}\right) \mathcal{H}^{1}\left(\mathcal{M}^{k} \cap \bar{\omega}\right) .
$$

Then the conclusion follows for a suitable diagonal sequence as already pursued in the proof of Theorem 4.11, Step 3.

Remark 5.8. Given $\varepsilon_{n} \rightarrow 0^{+}$, a slight modification of the above arguments yields that for every $(u, b) \in \mathscr{C}$, there is a sequence $\left\{\left(u_{n}, b_{n}\right)\right\} \subset$ $H^{2}\left(\omega ; \mathbb{R}^{3}\right) \times H^{1}\left(\omega ; \mathbb{R}^{3}\right)$ such that $\left(u_{n}, b_{n}\right) \rightarrow(u, b)$ in $W^{1, p}\left(\omega ; \mathbb{R}^{3}\right) \times L^{p}\left(\omega ; \mathbb{R}^{3}\right)$ and $\lim _{n} F_{\varepsilon_{n}}^{0}\left(u_{n}, b_{n}, \omega\right)=K_{0} \operatorname{Per}_{\omega}(E)$ where $\left(\nabla^{\prime} u, b\right)=\left(1-\chi_{E}\right) A+\chi_{E} B$, and $F_{\varepsilon_{n}}^{0}$ is defined by $(5.1)$.

Remark 5.9. Let us consider an arbitrary sequence $h_{n} \rightarrow 0^{+}$and $\varepsilon>0$ fixed. It is well known (see [9]) that the functionals $\left\{\mathscr{F}_{\varepsilon}^{h_{n}}\right\} \Gamma$-converge for the strong $L^{1}$-topology to

$\mathscr{F}_{\varepsilon}^{0}(u, b):= \begin{cases}F_{\varepsilon}^{0}(u, b, \omega) & \text { if }(u, b) \in H^{2}\left(\Omega: \mathbb{R}^{3}\right) \times H^{1}\left(\Omega ; \mathbb{R}^{3}\right) \quad \text { and } \quad \partial_{3} u=\partial_{3} b=0, \\ +\infty & \text { otherwise }\end{cases}$

where $F_{\varepsilon_{n}}^{0}$ is defined by $(5.1)$, and we have identified functions $(u, b)$ satisfying $\partial_{3} u=\partial_{3} b=0$ with functions defined on the mid-surface $\omega$. Let us now consider an arbitrary sequence $\varepsilon_{n} \rightarrow 0^{+}$. By Remarks 5.4 and 5.8 , the functionals $\left\{\mathscr{F}_{\varepsilon_{n}}^{0}\right\}$ in turn $\Gamma$-converge for the strong $L^{1}$-topology to $\mathscr{F}_{0}$ (compactness follows as in Theorem 1.1 with minor modifications).

\section{6. $\Gamma$-convergence in the supercritical regime}

This section is essentially devoted to the proof of Theorem 1.4. The $\Gamma$-liminf inequality is a direct consequence of the results in Sect. 4.1 once we have proved that under assumption $(1.9), K_{\infty}^{\star}<+\infty$. In contrast with the lower inequality, the estimate for the $\Gamma$-limsup requires a more sophisticated construction based on an homogenization procedure. The $\Gamma$-lim inf and $\Gamma$-limsup inequalities are stated in Theorems 6.2 and 6.7 respectively, and the conclusion follows from Lemma 6.1 . For $p=2, \lambda=0$, and under the symmetry assumption on $W$, we obtain the $\Gamma$-convergence of the functionals through Corollary 6.6. In a last subsection, we consider the situation where the wells $A$ and $B$ are not compatible, and we illustrate some rigidity phenomena in Theorems 6.9 and 6.10 . 


\subsection{The $\Gamma$-lim inf inequality}

We define the constant $K_{\infty}^{\star}$ as in (4.1) with $\gamma=+\infty$, i.e.,

$$
\begin{gathered}
K_{\infty}^{\star}:=\inf \left\{\liminf _{n \rightarrow \infty} F_{\varepsilon_{n}}^{h_{n}}\left(u_{n}, Q\right): h_{n} \rightarrow 0^{+} \text {and } \varepsilon_{n} \rightarrow 0^{+} \text {with } h_{n} / \varepsilon_{n} \rightarrow \infty,\right. \\
\left.\left\{u_{n}\right\} \subset H^{2}\left(Q ; \mathbb{R}^{3}\right),\left(u_{n}, \frac{1}{h_{n}} \partial_{3} u_{n}\right) \rightarrow\left(u_{0}, b_{0}\right) \text { in }\left[L^{1}\left(Q ; \mathbb{R}^{3}\right)\right]^{2}\right\} .
\end{gathered}
$$

We start by proving that if (1.9) holds, then $K_{\infty}^{\star}$ is finite and strictly positive.

Lemma 6.1. Assume that $\left(H_{1}\right)-\left(H_{3}\right)$ and (1.9) hold for some $\lambda \in \mathbb{R}$. Then $0<K_{\infty}^{\star}<\infty$.

Proof. Let us consider arbitrary sequences $\varepsilon_{n} \rightarrow 0^{+}, h_{n} \rightarrow 0^{+}$such that $h_{n} / \varepsilon_{n} \rightarrow \infty$. Observe that under assumption (1.9), we have $A=-B=$ $(a, 0, \lambda a)$ so that $A-B$ is rank-1 connected. By the results in [16], there exists a sequence $\left\{w_{n}\right\} \subset H^{2}\left((-1,1) ; \mathbb{R}^{3}\right)$ such that $w_{n} \rightarrow \bar{u}_{0}$ in $W^{1, p}\left((-1,1) ; \mathbb{R}^{3}\right)$, and

$$
\sup _{n \in \mathbb{N}} \int_{-1}^{1} \frac{1}{\varepsilon_{n}} \min \left\{\left|w_{n}^{\prime}-a\right|^{p},\left|w_{n}^{\prime}+a\right|^{p}\right\}+\varepsilon_{n}\left|w_{n}^{\prime \prime}\right|^{2} d t<\infty .
$$

For $n$ large enough, we consider the sequence $\left\{u_{n}\right\} \subset H^{2}\left(Q ; \mathbb{R}^{3}\right)$ defined by $u_{n}(x):=w_{n}\left(x_{1}+\lambda h_{n} x_{3}\right)$. Then one may check that $\left(u_{n}, \frac{1}{h_{n}} \partial_{3} u_{n}\right) \rightarrow\left(u_{0}, b_{0}\right)$ in $\left[L^{1}\left(Q ; \mathbb{R}^{3}\right)\right]^{2}$. Using Lemma 4.8 , we estimate

$F_{\varepsilon_{n}}^{h_{n}}\left(u_{n}, Q\right) \leqslant C \int_{-1}^{1} \frac{\left(1+\lambda^{2}\right)^{p / 2}}{\varepsilon_{n}} \min \left\{\left|w_{n}^{\prime}-a\right|^{p},\left|w_{n}^{\prime}+a\right|^{p}\right\}+\varepsilon_{n}\left(1+\lambda^{2}\right)^{2}\left|w_{n}^{\prime \prime}\right|^{2} d t$,

which shows that $\sup _{n} F_{\varepsilon_{n}}^{h_{n}}\left(u_{n}, Q\right)<\infty$, and thus $K_{\infty}^{*}<\infty$. On the other hand, we have

$$
\begin{array}{r}
K_{\infty}^{\star} \geqslant \inf \left\{\liminf _{n \rightarrow \infty} \int_{Q} \frac{1}{\varepsilon_{n}} W\left(\nabla^{\prime} u_{n}, b_{n}\right)+\varepsilon_{n}\left|\left(\nabla^{\prime}\right)^{2} u_{n}\right|^{2}+2 \varepsilon_{n}\left|\nabla^{\prime} b_{n}\right|^{2} d x^{\prime}: \varepsilon_{n} \rightarrow 0^{+},\right. \\
\left.\left\{\left(u_{n}, b_{n}\right)\right\} \subset H^{2}\left(Q ; \mathbb{R}^{3}\right) \times H^{1}\left(Q ; \mathbb{R}^{3}\right),\left(u_{n}, b_{n}\right) \rightarrow\left(u_{0}, b_{0}\right) \text { in }\left[L^{1}\left(Q ; \mathbb{R}^{3}\right)\right]^{2}\right\} .
\end{array}
$$

In view of Lemma 4.8 and [16, Sect. 5], we easily infer that

$$
\begin{gathered}
K_{\infty}^{\star} \geqslant \inf \left\{\liminf _{n \rightarrow \infty} \int_{I} \frac{1}{C_{*} \varepsilon_{n}} \min \left\{\left|v_{n}^{\prime}-a\right|^{p},\left|v_{n}^{\prime}+a\right|^{p}\right\}+\varepsilon_{n}\left|v_{n}^{\prime \prime}\right|^{2} d t: \varepsilon_{n} \rightarrow 0^{+},\right. \\
\left.\left\{v_{n}\right\} \subset H^{2}\left(I ; \mathbb{R}^{3}\right), v_{n} \rightarrow \bar{u}_{0} \text { in } L^{1}\left(I ; \mathbb{R}^{3}\right)\right\} \\
\geqslant \inf \left\{\int_{-L}^{L} \frac{1}{C_{*}} \min \left\{|v(t)-a|^{p},|v(t)+a|^{p}\right\}+\left|v^{\prime}\right|^{2} d t: L>0, v \text { piecewise } C^{1},\right. \\
v(L)=v(-L)=a\}>0,
\end{gathered}
$$

and the proof is complete. 
Thanks to Lemma 6.1 and Remark 4.5, we can now reproduce the first step in the proof of Theorem 4.6 to obtain the following result.

Theorem 6.2. Assume that $\left(H_{1}\right)-\left(H_{3}\right)$ and (1.9) hold for some $\lambda \in \mathbb{R}$. Let $h_{n} \rightarrow 0^{+}$and $\varepsilon_{n} \rightarrow 0^{+}$be arbitrary sequences such that $h_{n} / \varepsilon_{n} \rightarrow \infty$. Then, for any $(u, b) \in \mathscr{C}$ and any sequences $\left\{u_{n}\right\} \subset H^{2}\left(\Omega ; \mathbb{R}^{3}\right)$ such that $\left(u_{n}, \frac{1}{h_{n}} \partial_{3} u_{n}\right) \rightarrow$ $(u, b)$ in $\left[L^{1}\left(\Omega ; \mathbb{R}^{3}\right)\right]^{2}$, we have

$$
\liminf _{n \rightarrow \infty} F_{\varepsilon_{n}}^{h_{n}}\left(u_{n}\right) \geqslant K_{\infty}^{\star} \operatorname{Per}_{\omega}(E),
$$

where $\left(\nabla^{\prime} u, b\right)(x)=\left(1-\chi_{E}\left(x^{\prime}\right)\right) A+\chi_{E}\left(x^{\prime}\right) B$.

\subsection{Lower bound on $K_{\infty}^{\star}$ in the case $\lambda=0$}

As a direct consequence of Lemma 4.8, we have the following elementary property in the case $\lambda=0$.

Lemma 6.3. Assume that $\left(H_{1}\right)-\left(H_{3}\right)$ and (1.9) hold with $\lambda=0$. Then there is a constant $C_{W}>0$ such that $W(\xi) \geqslant C_{W}\left|\xi_{3}\right|^{p}$ for all $\xi=\left(\xi_{1}, \xi_{2}, \xi_{3}\right) \in \mathbb{R}^{3 \times 3}$.

In parallel with Proposition 4.9, the next propositions will establish that realizing sequences for $K_{\infty}^{\star}$ can be first chosen with lateral boundary conditions, and then periodic in the vertical direction.

Proposition 6.4. Assume that $\left(H_{1}\right)-\left(H_{4}\right)$ and (1.9) hold with $\lambda=0$. Then there exist sequences $h_{n} \rightarrow 0^{+}, \varepsilon_{n} \rightarrow 0,\left\{c_{n}\right\} \subset \mathbb{R}^{3}$, and $\left\{g_{n}\right\} \subset C^{2}\left(Q ; \mathbb{R}^{3}\right)$ such that $h_{n} / \varepsilon_{n} \rightarrow \infty, g_{n}$ is independent of $x_{2}$ (i.e., $g_{n}(x)=: \hat{g}_{n}\left(x_{1}, x_{3}\right)$ ), $c_{n} \rightarrow 0, g_{n} \rightarrow u_{0}$ in $W^{1, p}\left(Q ; \mathbb{R}^{3}\right), \frac{1}{h_{n}} \partial_{3} g_{n} \rightarrow 0$ in $L^{p}\left(Q ; \mathbb{R}^{3}\right)$,

$$
g_{n}=u_{0} \quad \text { in } Q \cap\left\{x_{1}>1 / 4\right\}, \quad g_{n}=u_{0}+c_{n} \text { in } Q \cap\left\{x_{1}<-1 / 4\right\}
$$

and $\lim _{n} F_{\varepsilon_{n}}^{h_{n}}\left(g_{n}, Q\right)=K_{\infty}^{\star}$.

Proof. Step 1. Since $\lambda=0$ we have $b_{0}=0$, and in view of Lemma 6.1 , there exist sequences $h_{n} \rightarrow 0^{+}, \varepsilon_{n} \rightarrow 0^{+}$and $\left\{u_{n}\right\} \subset H^{2}\left(Q ; \mathbb{R}^{3}\right)$ such that $h_{n} / \varepsilon_{n} \rightarrow$ $\infty,\left(u_{n}, \frac{1}{h_{n}} \partial_{3} u_{n}\right) \rightarrow\left(u_{0}, 0\right)$ in $\left[L^{1}\left(Q ; \mathbb{R}^{3}\right)\right]^{2}$, and $\lim _{n} F_{\varepsilon_{n}}^{h_{n}}\left(u_{n}, Q\right)=K_{\infty}^{\star}<$ $\infty$. Arguing as in the proof of Proposition 4.9, we may assume that $u_{n} \in$ $C^{2}\left(Q ; \mathbb{R}^{3}\right)$, and that $u_{n}$ is independent of $x_{2}$, i.e., $u_{n}(x)=: \hat{u}_{n}\left(x_{1}, x_{3}\right)$. By Theorem 1.1, $u_{n} \rightarrow u_{0}$ in $W^{1, p}\left(Q ; \mathbb{R}^{3}\right)$, and $\frac{1}{h_{n}} \partial_{3} u_{n} \rightarrow 0$ in $L^{p}\left(Q ; \mathbb{R}^{3}\right)$.

Step 2 (first matching). As in the proof of Proposition 4.9 we consider a partition of $\left(\frac{1}{12}, \frac{1}{6}\right) \times Q^{\prime}$ into $M_{n}:=\left[\frac{1}{\varepsilon_{n}}\right]$ layers along the $x_{1}$-direction. By Lemma 6.1 and Remark 4.5, we can find such a layer $L_{n}:=\left(\theta_{n}-\frac{1}{12 M_{n}}, \theta_{n}\right) \times$ $Q^{\prime} \subset\left(\frac{1}{12}, \frac{1}{6}\right) \times Q^{\prime}$ such that (4.8) holds (with $b_{0}=0$ ). Then select a level $t_{n} \in\left(\theta_{n}-\frac{1}{12 M_{n}}, \theta_{n}\right)$ for which (4.9) holds. We consider a cut-off function $\varphi_{n} \in C^{\infty}(\mathbb{R})$ satisfying (4.10), and we set for $x \in L_{n}$,

$$
v_{n}(x):=\left(1-\varphi_{n}\left(x_{1}\right)\right)\left(\bar{u}_{0}\left(x_{1}\right)+\bar{u}_{n}\left(x_{3}\right)\right)+\varphi_{n}\left(x_{1}\right) u_{n}(x),
$$

with $\bar{u}_{n}\left(x_{3}\right):=\hat{u}_{n}\left(t_{n}, x_{3}\right)-\bar{u}_{0}\left(t_{n}\right)$.

We claim that estimates (4.11)-(4.15) still hold (with $\left.b_{0}=0\right)$. First note that (4.11) is an easy consequence of (4.8) and (4.9). In view of Lemma 6.3, 
we infer from (4.9) that

$$
\frac{1}{\varepsilon_{n}} \int_{I} \frac{1}{h_{n}^{p}}\left|\bar{u}_{n}^{\prime}\left(x_{3}\right)\right|^{p} d x_{3}+\varepsilon_{n} \int_{I} \frac{1}{h_{n}^{4}}\left|\bar{u}_{n}^{\prime \prime}\left(x_{3}\right)\right|^{2} d x_{3} \leqslant C \alpha_{n} .
$$

Combining (4.8) and (6.2) yields (4.12). By construction $u_{n}-u_{0}-\bar{u}_{n}=0$ on $\left\{x_{1}=t_{n}\right\} \cap Q$, and applying Poincaré's inequality we deduce from (4.8),

$$
\int_{L_{n}}\left|u_{n}-u_{0}-\bar{u}_{n}\right|^{p} d x \leqslant C\left(\frac{1}{M_{n}}\right)^{p} \int_{L_{n}}\left|\partial_{1} u_{n}-\partial_{1} u_{0}\right|^{p} d x \leqslant C \alpha_{n} \varepsilon_{n}^{p+1} .
$$

Using (4.10), we may now infer that

$$
\frac{1}{\varepsilon_{n}} \int_{L_{n}}\left|\nabla^{\prime} v_{n}-\nabla^{\prime} u_{0}\right|^{p} d x \leqslant \frac{C}{\varepsilon_{n}} \int_{L_{n}}\left|\partial_{1} u_{n}-\partial_{1} u_{0}\right|^{p}+\frac{1}{\varepsilon_{n}^{p}}\left|u_{n}-u_{0}-\bar{u}_{n}\right|^{p} d x \leqslant C \alpha_{n} \rightarrow 0 .
$$

Estimates (4.12) and (4.13) being proved, (4.14) now follows exactly as in (4.19).

Using again (4.10), we estimate

$$
\begin{aligned}
& \varepsilon_{n} \int_{L_{n}}\left|\nabla_{h_{n}}^{2} v_{n}\right|^{2} d x \leqslant C\left(\varepsilon_{n} \int_{L_{n}}\left|\nabla_{h_{n}}^{2} u_{n}\right|^{2} d x+\frac{1}{\varepsilon_{n}} \int_{L_{n}}\left|\nabla^{\prime} u_{n}-\nabla^{\prime} u_{0}\right|^{2} d x\right. \\
& \quad+\frac{1}{\varepsilon_{n}^{3}} \int_{L_{n}}\left|u_{n}-u_{0}-\bar{u}_{n}\right|^{2} d x+\frac{1}{\varepsilon_{n}} \int_{L_{n}}\left|\frac{1}{h_{n}} \partial_{3} u_{n}\right|^{2} d x+\int_{I} \frac{1}{h_{n}^{2}}\left|\bar{u}_{n}^{\prime}\left(x_{3}\right)\right|^{2} d x_{3} \\
& \left.\quad+\varepsilon_{n}^{2} \int_{I} \frac{1}{h_{n}^{4}}\left|\bar{u}_{n}^{\prime \prime}\left(x_{3}\right)\right|^{2} d x_{3}\right),
\end{aligned}
$$

Arguing as in the proof of Proposition 4.9, Step 2, (4.15) now follows from (4.8), (6.2)-(6.4) together with Hölder's inequality.

Step 3 (second matching). Let $\psi_{n} \in C^{\infty}(\mathbb{R})$ be a cut-off function such that $0 \leqslant \psi_{n} \leqslant 1, \psi_{n}(t)=1$ if $t \leqslant \theta_{n}, \psi_{n}(t)=0$ if $t \geqslant 1 / 4$, and satisfying $\left|\psi_{n}^{\prime}\right|+\left|\psi_{n}^{\prime \prime}\right| \leqslant C$ for a constant $C$ independent of $n$. For $x \in\left\{\theta_{n}<x_{1}<\frac{1}{4}\right\} \cap Q$, we set

$$
w_{n}(x):=u_{0}(x)+c_{n}^{+}+\psi_{n}\left(x_{1}\right)\left(\bar{u}_{n}\left(x_{3}\right)-c_{n}^{+}\right),
$$

where $c_{n}^{+}:=\int_{I} \bar{u}_{n} d x_{3} \rightarrow 0$, thanks to (4.9). We claim that (4.20)-(4.23) hold.

First (4.20) and (4.21) are direct consequences of (4.9) and (6.2) respectively. Next we apply (6.2) and Poincaré's inequality to derive that

$$
\frac{1}{\varepsilon_{n}} \int_{\left\{\theta_{n}<x_{1}<\frac{1}{6}\right\} \cap Q}\left|\nabla^{\prime} w_{n}-\nabla^{\prime} u_{0}\right|^{p} d x \leqslant \frac{C}{\varepsilon_{n}} \int_{I}\left|\bar{u}_{n}\left(x_{3}\right)-c_{n}^{+}\right|^{p} d x_{3} \leqslant C h_{n}^{p} \alpha_{n} \rightarrow 0 .
$$

To prove (4.22), we can argue exactly as in (4.19) using (4.21) and (6.5).

We finally obtain in much similar ways that

$\varepsilon_{n} \int_{\left(\theta_{n}, \frac{1}{4}\right) \times Q^{\prime}}\left|\nabla_{h_{n}}^{2} w_{n}\right|^{2} d x \leqslant C \varepsilon_{n} \int_{I}\left|\bar{u}_{n}-c_{n}^{+}\right|^{2}+\frac{1}{h_{n}^{2}}\left|\bar{u}_{n}^{\prime}\right|^{2}+\frac{1}{h_{n}^{4}}\left|\bar{u}_{n}^{\prime \prime}\right|^{2} d x_{3} \leqslant C \alpha_{n} \rightarrow 0$, and (4.23) is proved. 
Step 4. We conclude the proof as in Proposition 4.9, Step 4. We first define $g_{n}^{+}$as in $(4.25)$ (with $b_{0}=0$ ), and then we repeat the procedure to modify $g_{n}^{+}$in $\left(-\frac{1}{2}, 0\right) \times Q^{\prime}$. We omit further details.

We now prove that, in the case where $p=2, \lambda=0$, and $W$ is symmetric in $\xi_{3}$, optimal sequences for $K_{\infty}^{\star}$ can be modified into 1-periodic functions in the $x_{3}$-variable without increasing the energy.

Proposition 6.5. (vertical periodicity) Assume that $\left(H_{1}\right)-\left(H_{4}\right)$ and (1.9) hold with $p=2, \lambda=0$, and that $W\left(\xi^{\prime}, \xi_{3}\right)=W\left(\xi^{\prime},-\xi_{3}\right)$ for every $\left(\xi^{\prime}, \xi_{3}\right) \in \mathbb{R}^{3 \times 2} \times$ $\mathbb{R}^{3}$. Then there exist sequences $h_{n} \rightarrow 0^{+}, \varepsilon_{n} \rightarrow 0^{+}$, and $\left\{f_{n}\right\} \subset C^{2}\left(\mathbb{R}^{3} ; \mathbb{R}^{3}\right)$ such that $h_{n} / \varepsilon_{n} \rightarrow \infty, f_{n}$ is independent of $x_{2}$ (i.e., $f_{n}(x)=\hat{f}_{n}\left(x_{1}, x_{3}\right)$ ), $f_{n} \rightarrow u_{0}$ in $H^{1}\left(Q ; \mathbb{R}^{3}\right), \frac{1}{h_{n}} \partial_{3} f_{n} \rightarrow 0$ in $L^{2}\left(Q ; \mathbb{R}^{3}\right), f_{n}$ is 1-periodic in the $x_{3}$ variable, $\nabla f_{n}=\nabla u_{0}$ in $\left\{\left|x_{1}\right|>1 / 4\right\}$, and

$$
\lim _{n \rightarrow \infty} F_{\varepsilon_{n}}^{h_{n}}\left(f_{n}, Q\right)=K_{\infty}^{\star} .
$$

Proof. Step 1. We claim that it suffices to find sequences $h_{n} \rightarrow 0^{+}, \varepsilon_{n} \rightarrow 0^{+}$, and $\left\{g_{n}^{\sharp}\right\} \subset C^{2}\left(\mathbb{R}^{3} ; \mathbb{R}^{3}\right)$ such that $h_{n} / \varepsilon_{n} \rightarrow \infty, g_{n}^{\sharp}(x)=: \hat{g}_{n}^{\sharp}\left(x_{1}, x_{3}\right), \nabla g_{n}^{\sharp}=\nabla u_{0}$ in $\left\{\left|x_{1}\right|>1 / 4\right\}, g_{n}^{\sharp}$ is 2-periodic in $x_{3}, g_{n}^{\sharp} \rightarrow u_{0}$ in $H^{1}\left(Q ; \mathbb{R}^{3}\right), \frac{1}{h_{n}} \partial_{3} g_{n}^{\sharp} \rightarrow 0$ in $L^{2}\left(Q ; \mathbb{R}^{3}\right)$, and $\lim \sup _{n} F_{\varepsilon_{n}}^{h_{n}}\left(g_{n}^{\sharp}, 2 Q\right) \leqslant 4 K_{\infty}^{\star}$. Indeed, if the claim holds we set $f_{n}(x):=\frac{1}{2} g_{n}^{\sharp}(2 x)$ for $x \in \mathbb{R}^{3}$. Then $f_{n} \rightarrow u_{0}$ in $H^{1}\left(Q ; \mathbb{R}^{3}\right), \frac{1}{h_{n}} \partial_{3} f_{n} \rightarrow 0$ in $L^{2}\left(Q ; \mathbb{R}^{3}\right)$. By definition of $K_{\infty}^{\star}$, a change of variables yields

$K_{\infty}^{\star} \leqslant \liminf _{n \rightarrow \infty} F_{\frac{\varepsilon_{n}}{2}}^{h_{n}}\left(f_{n}, Q\right) \leqslant \limsup _{n \rightarrow \infty} F_{\frac{\varepsilon_{n}}{2}}^{h_{n}}\left(f_{n}, Q\right)=\limsup _{n \rightarrow \infty} \frac{1}{4} F_{\varepsilon_{n}}^{h_{n}}\left(g_{n}^{\sharp}, 2 Q\right) \leqslant K_{\infty}^{\star}$, and thus $\left\{f_{n}\right\}$ satisfies the requirements (with $\varepsilon_{n} / 2$ instead of $\varepsilon_{n}$ ).

Step 2. Let $h_{n} \rightarrow 0^{+}$and $\varepsilon_{n} \rightarrow 0^{+}$satisfying $h_{n} / \varepsilon_{n} \rightarrow \infty$. Consider an arbitrary sequence $\left\{u_{n}\right\} \subset H^{2}\left(Q ; \mathbb{R}^{3}\right)$ such that $\left(u_{n}, \frac{1}{h_{n}} \partial_{3} u_{n}\right) \rightarrow\left(u_{0}, b_{0}\right)$ in $\left[L^{1}\left(Q ; \mathbb{R}^{3}\right)\right]^{2}$, and $\lim _{n} F_{\varepsilon_{n}}^{h_{n}}\left(u_{n}, Q\right)=K_{\infty}^{\star}$. We claim that for any $0<\delta<1 / 2$, we have

$$
\limsup _{n \rightarrow \infty} F_{\varepsilon_{n}}^{h_{n}}\left(u_{n}, Q^{\prime} \times((1 / 2-\delta, 1 / 2) \cup(-1 / 2,-1 / 2+\delta))\right) \leqslant 2 \delta K_{\infty}^{\star} .
$$

This is of course equivalent to the following inequality,

$$
\liminf _{n \rightarrow \infty} F_{\varepsilon_{n}}^{h_{n}}\left(u_{n}, Q^{\prime} \times(-1 / 2+\delta, 1 / 2-\delta)\right) \geqslant(1-2 \delta) K_{\infty}^{\star},
$$

that we prove by rescaling. For $x \in Q$, we set $v_{n}(x):=u_{n}\left(x^{\prime},(1-2 \delta) x_{3}\right)$ and $\tilde{h}_{n}:=(1-2 \delta) h_{n}$. Then $\tilde{h}_{n} / \varepsilon_{n} \rightarrow \infty$ and $\left(v_{n}, \frac{1}{\tilde{h}_{n}} \partial_{3} v_{n}\right) \rightarrow\left(u_{0}, b_{0}\right)$ in $\left[L^{1}\left(Q ; \mathbb{R}^{3}\right)\right]^{2}$. Therefore,

$K_{\infty}^{\star} \leqslant \liminf _{n \rightarrow \infty} F_{\varepsilon_{n}}^{h_{n}}\left(v_{n}, Q\right)=\liminf _{n \rightarrow \infty} \frac{1}{1-2 \delta} F_{\varepsilon_{n}}^{h_{n}}\left(u_{n}, Q^{\prime} \times(-1 / 2+\delta, 1 / 2-\delta)\right)$,

and (6.6) follows.

Step 3. Consider the sequences $\left\{h_{n}\right\},\left\{\varepsilon_{n}\right\}$, and $\left\{g_{n}\right\} \subset C^{2}\left(Q ; \mathbb{R}^{3}\right)$ given by Proposition 6.4, and let us fix $m \in \mathbb{N}$ arbitrarily large. We infer from Step 2 (with $\delta=1 / m$ ) that

$$
\limsup _{n \rightarrow \infty} F_{\varepsilon_{n}}^{h_{n}}\left(g_{n}, Q \cap\left\{\frac{1}{2}-\frac{1}{m}<\left|x_{3}\right|<\frac{1}{2}\right\}\right) \leqslant \frac{2}{m} K_{\infty}^{\star} .
$$


Next we divide $Q^{\prime} \times\left(\frac{1}{2}-\frac{1}{m}, \frac{1}{2}\right)$ into $\left[\frac{h_{n}}{\varepsilon_{n}}\right]$ thin horizontal strips $R_{m, n, i}^{+}$of width $\frac{1}{m}\left[\frac{h_{n}}{\varepsilon_{n}}\right]^{-1}$, i.e.,

$$
R_{m, n, i}^{+}:=Q^{\prime} \times\left(\frac{1}{2}-\frac{i}{m}\left[\frac{h_{n}}{\varepsilon_{n}}\right]^{-1}, \frac{1}{2}-\frac{i-1}{m}\left[\frac{h_{n}}{\varepsilon_{n}}\right]^{-1}\right)
$$

for $i=1, \ldots,\left[\frac{h_{n}}{\varepsilon_{n}}\right]$. We proceed symmetrically in the set $Q^{\prime} \times\left(-\frac{1}{2},-\frac{1}{2}+\frac{1}{m}\right)$, and we denote by $R_{m, n, i}^{-}$the resulting strips. Applying Lemma 6.3, we infer from (6.7) that for $n$ large enough,

$$
\begin{aligned}
& \sum_{i=1}^{\left[\frac{h_{n}}{\varepsilon_{n}}\right]} \int_{R_{m, n, i}^{-} \cup R_{m, n, i}^{+}}\left(\frac{1}{\varepsilon_{n}} W\left(\nabla_{h_{n}} g_{n}\right)+\varepsilon_{n}\left|\nabla_{h_{n}}^{2} g_{n}\right|^{2}+\left|\nabla^{\prime} g_{n}-\nabla^{\prime} u_{0}\right|^{2}\right. \\
& \left.\quad+\frac{C_{W}}{\varepsilon_{n}}\left|\frac{1}{h_{n}} \partial_{3} g_{n}\right|^{2}+\left|g_{n}-u_{0}\right|^{2}\right) d x \leqslant \frac{4}{m} K_{\infty}^{\star} .
\end{aligned}
$$

where we also have used the fact that $\left\|g_{n}-u_{0}\right\|_{H^{1}(Q)} \rightarrow 0$. Now consider a pair of strips $\left(R_{m, n, i_{0}}^{-}, R_{m, n, i_{0}}^{+}\right)$with $i_{0}=i_{0}(m, n)$ satisfying

$$
\begin{gathered}
\int_{R_{m, n, i_{0}}^{-} \cup R_{m, n, i_{0}}^{+}}\left(\frac{1}{\varepsilon_{n}} W\left(\nabla_{h_{n}} g_{n}\right)+\varepsilon_{n}\left|\nabla_{h_{n}}^{2} g_{n}\right|^{2}+\left|\nabla^{\prime} g_{n}-\nabla^{\prime} u_{0}\right|^{2}\right. \\
\left.+\frac{C_{W}}{\varepsilon_{n}}\left|\frac{1}{h_{n}} \partial_{3} g_{n}\right|^{2}+\left|g_{n}-u_{0}\right|^{2}\right) d x \leqslant \frac{4}{m}\left[\frac{h_{n}}{\varepsilon_{n}}\right]^{-1} K_{\infty}^{\star},
\end{gathered}
$$

and we shall write for simplicity $R_{m, n}^{ \pm}:=R_{m, n, i_{0}}^{ \pm}$(respectively). Then we choose a level

$$
t_{m, n} \in\left(\frac{1}{2}-\frac{i_{0}-1 / 2}{m}\left[\frac{h_{n}}{\varepsilon_{n}}\right]^{-1}, \frac{1}{2}-\frac{i_{0}-1}{m}\left[\frac{h_{n}}{\varepsilon_{n}}\right]^{-1}\right)
$$

for which

$$
\begin{aligned}
& \int_{Q \cap\left\{\left|x_{3}\right|=t_{m, n}\right\}}\left(\frac{1}{\varepsilon_{n}} W\left(\nabla_{h_{n}} g_{n}\right)+\varepsilon_{n}\left|\nabla_{h_{n}}^{2} g_{n}\right|^{2}+\left|\nabla^{\prime} g_{n}-\nabla^{\prime} u_{0}\right|^{2}\right. \\
& \left.+\frac{C_{W}}{\varepsilon_{n}}\left|\frac{1}{h_{n}} \partial_{3} g_{n}\right|^{2}+\left|g_{n}-u_{0}\right|^{2}\right) d \mathcal{H}^{2} \leqslant 8 K_{\infty}^{\star} .
\end{aligned}
$$

Let $\varphi_{m, n}: \mathbb{R} \rightarrow[0,1]$ be a smooth cut-off function such that $\varphi_{m, n}(t)=0$ for $t>t_{m, n}, \varphi_{m, n}(t)=1$ for $t<t_{m, n}-\frac{1}{2 m}\left[\frac{h_{n}}{\varepsilon_{n}}\right]^{-1}$, and

$$
\frac{\varepsilon_{n}}{m h_{n}}\left|\varphi_{m, n}^{\prime}\right|+\frac{\varepsilon_{n}^{2}}{m^{2} h_{n}^{2}}\left|\varphi_{m, n}^{\prime \prime}\right| \leqslant C,
$$

for a constant $C$ independent of $m$ and $n$. We define for $x \in Q$,

$$
w_{m, n}(x):=\varphi_{m, n}\left(x_{3}\right) g_{n}(x)+\left(1-\varphi_{m, n}\left(x_{3}\right)\right) \hat{g}_{n}\left(x_{1}, t_{m, n}\right) .
$$

We shall prove in Step 4 below that

$$
\limsup _{m \rightarrow \infty} \limsup _{n \rightarrow \infty}\left\|w_{m, n}-u_{0}\right\|_{H^{1}(Q)}+\left\|\frac{1}{h_{n}} \partial_{3} w_{m, n}\right\|_{L^{2}(Q)}=0
$$


and

$$
\limsup _{m \rightarrow \infty} \limsup _{n \rightarrow \infty} F_{\varepsilon_{n}}^{h_{n}}\left(w_{m, n}, Q\right) \leqslant K_{\infty}^{\star} .
$$

Assuming for the moment that (6.11) and (6.12) hold, we find a diagonal sequence $n_{m} \rightarrow+\infty$ such that setting $\varepsilon_{m}:=\varepsilon_{n_{m}}, h_{m}:=h_{n_{m}}$, and $w_{m}:=$ $w_{m, n_{m}}$, we have $w_{m} \rightarrow u_{0}$ in $H^{1}\left(Q ; \mathbb{R}^{3}\right), \frac{1}{h_{m}} \partial_{3} w_{m} \rightarrow 0$ in $L^{2}\left(Q ; \mathbb{R}^{3}\right)$, and $\lim \sup _{m} F_{\varepsilon_{m}}^{h_{m}}\left(w_{m}, Q\right) \leqslant K_{\infty}^{\star}$. We now repeat this construction in the strip $R_{m, n}^{-}$, and we write $\tilde{w}_{m}$ the resulting function.

Since $\tilde{w}_{m}$ is independent of $x_{3}$ in a neighborhood of $\left\{\left|x_{3}\right|=1 / 2\right\} \cap Q$, we may first reflect $\tilde{w}_{m}$ across the hyperplane $\left\{x_{3}=1 / 2\right\}$ setting for $\frac{1}{2} \leqslant$ $x_{3} \leqslant \frac{3}{2}, \tilde{w}_{m}\left(x^{\prime}, x_{3}\right):=w_{m}\left(x^{\prime}, 1-x_{3}\right)$, and then we extend $\tilde{w}_{m}$ by periodicity to all values of $x_{3}$. The resulting function $\tilde{w}_{m}$ belongs to $C^{2}\left(Q^{\prime} \times \mathbb{R} ; \mathbb{R}^{3}\right)$. Since $\nabla \tilde{w}_{m}=\nabla u_{0}$ in $\left\{\left|x_{1}\right|>1 / 4\right\}$, we can extend linearly $\tilde{w}_{m}$ in $x_{1}$, and constantly in $x_{2}$. We finally set for $x \in \mathbb{R}^{3}, g_{m}^{\sharp}(x):=\tilde{w}_{m}\left(x^{\prime}, x_{3}-\frac{1}{2}\right)$. Since $W\left(\xi^{\prime},-\xi_{3}\right)=W\left(\xi^{\prime}, \xi_{3}\right)$ for all $\xi \in \mathbb{R}^{3 \times 3}$, we find that

$$
F_{\varepsilon_{m}}^{h_{m}}\left(g_{m}^{\sharp}, 2 Q\right)=4 F_{\varepsilon_{m}}^{h_{m}}\left(\tilde{w}_{m}, Q\right),
$$

so that the function $g_{m}^{\sharp}$ satisfies all the requirements of Step 1 .

Step 4. We now complete the proof by showing that (6.11) and (6.12) do hold. To this purpose we shall write

$$
L_{m, n}^{+}:=Q^{\prime} \times\left(\frac{1}{2}-\frac{i_{0}}{m}\left[\frac{h_{n}}{\varepsilon_{n}}\right]^{-1}, \frac{1}{2}\right) .
$$

We first estimate

$$
\begin{aligned}
F_{\varepsilon_{n}}^{h_{n}}\left(w_{m, n}, Q\right)= & F_{\varepsilon_{n}}^{h_{n}}\left(g_{n}, Q \backslash L_{m, n}^{+}\right)+F_{\varepsilon_{n}}^{h_{n}}\left(w_{m, n}, R_{m, n}^{+}\right) \\
& +\int_{L_{m, n}^{+} \backslash R_{m, n}^{+}} \frac{1}{\varepsilon_{n}} W\left(\partial_{1} \hat{g}_{n}\left(x_{1}, t_{m, n}\right), 0,0\right)+\varepsilon_{n}\left|\partial_{1}^{2} \hat{g}_{n}\left(x_{1}, t_{m, n}\right)\right|^{2} d x \\
\leqslant & F_{\varepsilon_{n}}^{h_{n}}\left(g_{n}, Q\right)+F_{\varepsilon_{n}}^{h_{n}}\left(w_{m, n}, R_{m, n}^{+}\right) \\
& +\frac{1}{m} \int_{-1 / 2}^{1 / 2} \frac{1}{\varepsilon_{n}} W\left(\partial_{1} \hat{g}_{n}\left(x_{1}, t_{m, n}\right), 0,0\right)+\varepsilon_{n}\left|\partial_{1}^{2} \hat{g}_{n}\left(x_{1}, t_{m, n}\right)\right|^{2} d x_{1} .
\end{aligned}
$$

By Lemmas 4.8 and 6.3, we have

$$
\begin{aligned}
& W\left(\partial_{1} \hat{g}_{n}\left(x_{1}, t_{m, n}\right), 0,0\right) \leqslant C\left(W\left(\nabla_{h_{n}} g_{n}\left(x^{\prime}, t_{m, n}\right)\right)+\left|\frac{1}{h_{n}} \partial_{3} g_{n}\left(x^{\prime}, t_{m, n}\right)\right|^{2}\right) \\
& \quad \leqslant C W\left(\nabla_{h_{n}} g_{n}\left(x^{\prime}, t_{m, n}\right)\right),
\end{aligned}
$$

so that (6.9) yields

$$
\begin{aligned}
& \frac{1}{m} \int_{-1 / 2}^{1 / 2} \frac{1}{\varepsilon_{n}} W\left(\partial_{1} \hat{g}_{n}\left(x_{1}, t_{m, n}\right), 0,0\right)+\varepsilon_{n}\left|\partial_{1}^{2} \hat{g}_{n}\left(x_{1}, t_{m, n}\right)\right|^{2} d x_{1} \\
& \leqslant \frac{C}{m} \int_{Q \cap\left\{x_{3}=t_{m, n}\right\}} \frac{1}{\varepsilon_{n}} W\left(\nabla_{h_{n}} g_{n}\right)+\varepsilon_{n}\left|\nabla_{h_{n}}^{2} g_{n}\right|^{2} d \mathcal{H}^{2} \leqslant \frac{C}{m} .
\end{aligned}
$$


Similarly, we infer from (6.9) that

$$
\begin{aligned}
\int_{Q}\left|w_{m, n}-u_{0}\right|^{2}+\left|\frac{1}{h_{n}} \partial_{3} w_{m, n}\right|^{2} d x \\
\leqslant \int_{Q}\left|g_{n}-u_{0}\right|^{2}+\left|\frac{1}{h_{n}} \partial_{3} g_{n}\right|^{2} d x+\int_{R_{m, n}^{+}}\left|w_{m, n}-u_{0}\right|^{2}+\left|\frac{1}{h_{n}} \partial_{3} w_{m, n}\right|^{2} d x \\
\quad+\frac{1}{m} \int_{-1 / 2}^{1 / 2}\left|\hat{g}_{n}\left(x_{1}, t_{m, n}\right)-\bar{u}_{0}\left(x_{1}\right)\right|^{2} d x_{1} \\
\leqslant \int_{Q}\left|g_{n}-u_{0}\right|^{2}+\left|\frac{1}{h_{n}} \partial_{3} g_{n}\right|^{2} d x+\int_{R_{m, n}^{+}}\left|w_{m, n}-u_{0}\right|^{2}+\left|\frac{1}{h_{n}} \partial_{3} w_{m, n}\right|^{2} d x+\frac{C}{m} .
\end{aligned}
$$

In view of (6.13)-(6.15), and Theorem 1.1, to prove (6.11) and (6.12) it suffices to show that for every $m \in \mathbb{N}$ large enough,

$$
\lim _{n \rightarrow \infty} \int_{R_{m, n}^{+}}\left|w_{m, n}-u_{0}\right|^{2}+\left|\frac{1}{h_{n}} \partial_{3} w_{m, n}\right|^{2} d x=0
$$

and

$$
\lim _{n \rightarrow \infty} F_{\varepsilon_{n}}^{h_{n}}\left(w_{m, n}, R_{m, n}^{+}\right)=0 .
$$

We start with the proof of (6.17). Writing for $x \in R_{m, n}^{+}$,

$\partial_{1} w_{m, n}(x)=\partial_{1} g_{n}(x)+\left(1-\varphi_{m, n}\left(x_{3}\right)\right)\left(\partial_{1} \hat{g}_{n}\left(x_{1}, t_{m, n}\right)-\partial_{1} g_{n}(x)\right)$,

and

$$
\begin{aligned}
& \frac{1}{h_{n}} \partial_{3} w_{m, n}(x)=\frac{1}{h_{n}} \partial_{3} g_{n}(x)-\left(1-\varphi_{m, n}\left(x_{3}\right)\right) \frac{1}{h_{n}} \partial_{3} g_{n}(x) \\
& \quad+\frac{\varphi_{m, n}^{\prime}\left(x_{3}\right)}{h_{n}}\left(g_{n}(x)-\hat{g}_{n}\left(x_{1}, t_{m, n}\right)\right),
\end{aligned}
$$

we derive from Lemmas 4.8, 6.3, and (6.10) that

$$
\begin{aligned}
& W\left(\nabla_{h_{n}} w_{m, n}(x)\right) \leqslant C\left(W\left(\nabla_{h_{n}} g(x)\right)\right. \\
& \left.\quad+\left|\partial_{1} g_{n}(x)-\partial_{1} \hat{g}_{n}\left(x_{1}, t_{m, n}\right)\right|^{2}+\frac{m^{2}}{\varepsilon_{n}^{2}}\left|g_{n}(x)-\hat{g}_{n}\left(x_{1}, t_{m, n}\right)\right|^{2}\right) .
\end{aligned}
$$

Using Poincaré's inequality and (6.8), we estimate

$$
\frac{m^{2}}{\varepsilon_{n}^{3}} \int_{R_{m, n}^{+}}\left|g_{n}(x)-\hat{g}_{n}\left(x_{1}, t_{m, n}\right)\right|^{2} d x \leqslant C \frac{1}{\varepsilon_{n}} \int_{R_{m, n}^{+}}\left|\frac{1}{h_{n}} \partial_{3} g_{n}(x)\right|^{2} d x \leqslant \frac{C}{m} \frac{\varepsilon_{n}}{h_{n}},
$$

and

$\frac{1}{\varepsilon_{n}} \int_{R_{m, n}^{+}}\left|\partial_{1} g(x)-\partial_{1} \hat{g}_{n}\left(x_{1}, t_{m, n}\right)\right|^{2} d x \leqslant C \frac{\varepsilon_{n}}{m^{2} h_{n}^{2}} \int_{R_{m, n}^{+}}\left|\partial_{13}^{2} g(x)\right|^{2} d x \leqslant \frac{C}{m^{3}} \frac{\varepsilon_{n}}{h_{n}}$. 
In view of (6.18), we have thus obtained

$$
\frac{1}{\varepsilon_{n}} \int_{R_{m, n}^{+}} W\left(\nabla_{h_{n}} w_{m, n}\right) d x \leqslant C\left(\frac{1}{\varepsilon_{n}} \int_{R_{m, n}^{+}} W\left(\nabla_{h_{n}} g_{n}\right) d x+\frac{\varepsilon_{n}}{h_{n}}\right) \leqslant C \frac{\varepsilon_{n}}{h_{n}} \underset{n \rightarrow \infty}{\longrightarrow} 0 .
$$

Then, straightforward computations using (6.10) yield

$$
\begin{aligned}
\left|\nabla_{h_{n}}^{2} w_{m, n}(x)\right|^{2} \leqslant C & \left(\left|\nabla_{h_{n}}^{2} g_{n}(x)\right|^{2}+\left|\partial_{1}^{2} \hat{g}_{n}\left(x_{1}, t_{m, n}\right)\right|^{2}+\frac{m^{2}}{\varepsilon_{n}^{2}}\left|\partial_{1} g_{n}(x)-\partial_{1} \hat{g}_{n}\left(x_{1}, t_{m, n}\right)\right|^{2}\right. \\
& \left.+\frac{m^{2}}{\varepsilon_{n}^{2}}\left|\frac{1}{h_{n}} \partial_{3} g_{n}(x)\right|^{2}+\frac{m^{4}}{\varepsilon_{n}^{4}}\left|g_{n}(x)-\hat{g}_{n}\left(x_{1}, t_{m, n}\right)\right|^{2}\right) .
\end{aligned}
$$

Combining (6.8), (6.9), (6.19), and (6.20), we deduce that

$$
\varepsilon_{n} \int_{R_{m, n}^{+}}\left|\nabla_{h_{n}}^{2} w_{m, n}\right|^{2} d x \leqslant C m \frac{\varepsilon_{n}}{h_{n}} \underset{n \rightarrow \infty}{\longrightarrow} 0,
$$

which completes the proof of (6.17).

Using (6.8), (6.9), (6.19), we finally estimate

$$
\begin{aligned}
& \int_{R_{m, n}^{+}}\left|w_{m, n}-u_{0}\right|^{2}+\left|\frac{1}{h_{n}} \partial_{3} w_{m, n}\right|^{2} d x \leqslant C\left(\int_{R_{m, n}^{+}}\left|g_{n}-u_{0}\right|^{2}+\left|\frac{1}{h_{n}} \partial_{3} g_{n}\right|^{2} d x\right. \\
& \left.\quad+\frac{m^{2}}{\varepsilon_{n}^{2}} \int_{R_{m, n}^{+}}\left|g_{n}(x)-\hat{g}_{n}\left(x_{1}, t_{m, n}\right)\right|^{2} d x+\frac{\varepsilon_{n}}{h_{n}} \int_{Q \cap\left\{x_{3}=t_{m, n}\right\}}\left|g_{n}-u_{0}\right|^{2} d \mathcal{H}^{2}\right) \\
& \leqslant C \frac{\varepsilon_{n}}{h_{n}},
\end{aligned}
$$

and (6.16) is proved.

Corollary 6.6. Assume that $\left(H_{1}\right)-\left(H_{4}\right)$ and (1.9) hold with $p=2, \lambda=0$, and that $W\left(\xi^{\prime}, \xi_{3}\right)=W\left(\xi^{\prime},-\xi_{3}\right)$ for every $\xi=\left(\xi^{\prime}, \xi_{3}\right) \in \mathbb{R}^{3 \times 3}$. Then $K_{\infty}^{\star} \geqslant K_{\infty}$.

Proof. We consider the sequences $h_{n} \rightarrow 0^{+}, \varepsilon_{n} \rightarrow 0^{+}$, and $\left\{f_{n}\right\} \subset C^{2}\left(\mathbb{R}^{3} ; \mathbb{R}^{3}\right)$ given by Proposition 6.5. We define $N_{n}:=\left[\frac{1}{h_{n}}\right], \rho_{n}:=\frac{1}{N_{n} h_{n}}$, and $\ell_{n}:=\frac{1}{\rho_{n} \varepsilon_{n}}$ ([.] still denotes the integer part). Recalling that $f_{n}(x)=\hat{f}_{n}\left(x_{1}, x_{3}\right)$, we define for $y \in \mathbb{R}^{2}$,

$$
v_{n}(y):=\rho_{n} \hat{f}_{n}\left(\frac{y_{1}}{\rho_{n}}, \frac{y_{2}}{\rho_{n} h_{n}}\right) .
$$

Then $v_{n}$ is $1 / N_{n}$-periodic in the $y_{2}$-variable, and $\nabla v_{n}(y)=\left(\bar{u}_{0}\left(y_{1}\right), 0\right)$ in $\left\{\left|y_{1}\right|>\frac{\rho_{n}}{4}\right\}$. Since $v_{n}$ is $1 / N_{n}$-periodic in $y_{2}$, and $N_{n}$ being an integer, we deduce that $v_{n}$ is also 1 -periodic in $y_{2}$. Moreover, since $\rho_{n} \rightarrow 1$, we have for $n$ large enough

$$
\nabla v_{n}(y)=\left(\bar{u}_{0}\left(y_{1}\right), 0\right) \quad \text { in } \quad\left\{\left|y_{1}\right|>1 / 3\right\}
$$

Hence,

$$
\int_{Q^{\prime}} \ell_{n} \mathcal{W}\left(\nabla v_{n}\right)+\frac{1}{\ell_{n}}\left|\nabla^{2} v_{n}\right|^{2} d y \geqslant K_{\infty}
$$

Changing variables, using (6.21) and the 1 -periodicity in $x_{3}$ of $f_{n}$, we compute $\int_{Q^{\prime}} \ell_{n} \mathcal{W}\left(\nabla v_{n}\right)+\frac{1}{\ell_{n}}\left|\nabla^{2} v_{n}\right|^{2} d y=\rho_{n} h_{n} F_{\varepsilon_{n}}^{h_{n}}\left(f_{n}, Q^{\prime} \times N_{n} I\right)=F_{\varepsilon_{n}}^{h_{n}}\left(f_{n}, Q\right) \underset{n \rightarrow \infty}{\longrightarrow} K_{\infty}^{\star}$, 
which completes the proof.

\subsection{The $\Gamma$-lim sup inequality}

The next theorem provides the announced upper bound for the $\Gamma-\limsup$ of the functionals $\left\{F_{\varepsilon}^{h}\right\}$ when $\varepsilon \ll h$, and thus completing the proof of Theorem 1.4.

Theorem 6.7. Assume that $\left(H_{1}\right)-\left(H_{4}\right)$ and (1.9) hold for some $\lambda \in \mathbb{R}$. Let $\varepsilon_{n} \rightarrow 0^{+}$and $h_{n} \rightarrow 0^{+}$be arbitrary sequences such that $h_{n} / \varepsilon_{n} \rightarrow \infty$. Then, for every $(u, b) \in \mathscr{C}$, there exists a sequence $\left\{u_{n}\right\} \subset H^{2}\left(\Omega ; \mathbb{R}^{3}\right)$ such that $u_{n} \rightarrow u$ in $W^{1, p}\left(\Omega ; \mathbb{R}^{3}\right), \frac{1}{h_{n}} \partial_{3} u_{n} \rightarrow b$ in $L^{p}\left(\Omega ; \mathbb{R}^{3}\right)$, and

$$
\limsup _{n \rightarrow \infty} F_{\varepsilon_{n}}^{h_{n}}\left(u_{n}\right) \leqslant K_{\infty} \operatorname{Per}_{\omega}(E),
$$

where $\left(\nabla^{\prime} u, b\right)(x)=\left(1-\chi_{E}\left(x^{\prime}\right)\right) A+\chi_{E}\left(x^{\prime}\right) B$.

Proof. We first introduce some useful notation. For a given a sequence $h_{n} \rightarrow$ $0^{+}$, we define

$$
\nu_{n}:=\frac{e_{1}+\lambda h_{n} e_{3}}{\sqrt{1+\lambda^{2} h_{n}^{2}}} \in \mathbb{S}^{2} \quad \text { and } \quad \nu_{n}^{\perp}:=\frac{-\lambda h_{n} e_{1}+e_{3}}{\sqrt{1+\lambda^{2} h_{n}^{2}}} \in \mathbb{S}^{2} .
$$

We recall that $Q_{\lambda}^{\prime}$ denotes the unit cube of $\mathbb{R}^{2}$ centered at the origin with two faces orthogonal to the unit vector $\nu_{\lambda}=\frac{1}{\sqrt{1+\lambda^{2}}}(1, \lambda)$.

By Theorem 2.1 and (1.9), $\partial^{*} E \cap \omega$ is of the form (2.5). We assume that $\partial^{*} E \cap \omega$ is made by finitely many interfaces, i.e., $\mathscr{I}=\{1, \ldots, m\}$ in $(2.5)$. The proof for infinitely many interfaces follows from a diagonalization argument as in the proof of Theorem 4.11. Then $u(x)=\bar{u}\left(x_{1}\right)$ for some function $\bar{u}$ that we may assume to be as in the proof of Theorem 4.11, Step 1 (we refer to it for the notation). Then (1.9) yields $b(x)=\lambda \bar{u}^{\prime}\left(x_{1}\right)$.

Let us now consider for each $k \in \mathbb{N}$, some $\ell_{k}>0$ and some function $v_{k} \in C^{2}\left(\mathbb{R}^{2} ; \mathbb{R}^{3}\right)$ 1-periodic in the direction $\nu_{\lambda}^{\perp}:=\frac{1}{\sqrt{1+\lambda^{2}}}(-\lambda, 1)$, satisfying $\nabla v_{k}(y)= \pm(a, \lambda a)$ nearby $\left\{y \cdot \nu_{\lambda}= \pm 1 / 2\right\}$ respectively, and such that

$$
\int_{Q_{\lambda}^{\prime}} \ell_{k} \mathcal{W}\left(\nabla v_{k}\right)+\frac{1}{\ell_{k}}\left|\nabla^{2} v_{k}\right|^{2} d y \leqslant \frac{K_{\infty}+2^{-k}}{\sqrt{1+\lambda^{2}}} .
$$

Without loss of generality we may assume that

$$
v_{k}(y)=\left\{\begin{array}{l}
\sqrt{1+\lambda^{2}}\left(y \cdot \nu_{\lambda}\right) a+c_{k} \quad \text { nearby }\left\{y \cdot \nu_{\lambda}=1 / 2\right\}, \\
-\sqrt{1+\lambda^{2}}\left(y \cdot \nu_{\lambda}\right) a-c_{k} \text { nearby }\left\{y \cdot \nu_{\lambda}=-1 / 2\right\},
\end{array}\right.
$$

for some constant $c_{k} \in \mathbb{R}^{3}$. From now on we drop the subscript $k$ for simplicity.

Let $\varepsilon_{n} \rightarrow 0^{+}$and $h_{n} \rightarrow 0^{+}$be arbitrary sequences such that $h_{n} / \varepsilon_{n} \rightarrow$ $+\infty$. Again we choose for each index $i=1, \ldots, m$, an bounded open interval $J_{i}^{\prime} \subset \mathbb{R}$ such that $J_{i} \subset \subset J_{i}^{\prime}$ and $\mathcal{H}^{1}\left(J_{i}^{\prime} \backslash J_{i}\right) \leqslant 2^{-k}$. We write

$$
\alpha_{i \pm}^{n}:=\frac{1}{\sqrt{1+\lambda^{2} h_{n}^{2}}}\left(\alpha_{i} \pm \frac{\ell \varepsilon_{n} \sqrt{1+\lambda^{2}}}{2}\right)
$$


and we consider integers $n$ large enough in such a way that $\alpha_{i+}^{n}<\alpha_{(i+1)-}^{n}$ for every $i$, and for which (4.31) holds. We define the transition layers as follows: for $i=1, \ldots, m$ and for $x \in \mathbb{R}^{3}$, we set

$w_{n}^{i}(x):=(-1)^{i+1} v\left((-1)^{i+1} \frac{x_{1}-\alpha_{i}}{\ell \varepsilon_{n}},(-1)^{i+1} \frac{h_{n} x_{3}}{\ell \varepsilon_{n}}\right)+\left(1+(-1)^{i}\right)\left(\frac{1}{2} \sqrt{1+\lambda^{2}} a+c\right)$.

Then (6.23) yields

$$
w_{n}^{i}(x)=\frac{1}{2} \sqrt{1+\lambda^{2}} a-(-1)^{i+1} c \quad \text { on }\left\{x \cdot \nu_{n}=\alpha_{i-}^{n}\right\},
$$

and

$$
w_{n}^{i}(x)=\left(\frac{1}{2} \sqrt{1+\lambda^{2}} a+(-1)^{i+1} c\right)+2\left(1+(-1)^{i}\right) c \quad \text { on }\left\{x \cdot \nu_{n}=\alpha_{i+}^{n}\right\} .
$$

Setting

$$
\beta_{i}^{n}:=\sum_{j=1}^{i} \bar{u}\left(\alpha_{j+}^{n} \sqrt{1+\lambda^{2} h_{n}^{2}}\right)-\bar{u}\left(\alpha_{j-}^{n} \sqrt{1+\lambda^{2} h_{n}^{2}}\right),
$$

with $\beta_{0}^{n}:=0$ and $\kappa_{i}$ as in (4.34), we define for $n$ large enough and $x \in \Omega$,

$$
\begin{aligned}
& u_{n}(x) \\
& := \begin{cases}\bar{u}\left(x_{1}+\lambda h_{n} x_{3}\right)+\ell \varepsilon_{n}\left(\frac{a}{2} \sqrt{1+\lambda^{2}}-c\right) & \text { for } x \cdot \nu_{n} \leqslant \alpha_{1-}^{n} \\
\bar{u}\left(\alpha_{i-}^{n} \sqrt{1+\lambda^{2} h_{n}^{2}}\right)-\beta_{i-1}^{n}+\ell \varepsilon_{n}\left(w_{n}^{i}(x)+\kappa_{i-1} c\right) & \text { for } \alpha_{i-}^{n}<x \cdot \nu_{n}<\alpha_{i+}^{n} \\
\bar{u}\left(x_{1}+\lambda h_{n} x_{3}\right)-\beta_{i}^{n}+\ell \varepsilon_{n}\left(\frac{a}{2} \sqrt{1+\lambda^{2}}+\left((-1)^{i+1}+\kappa_{i}\right) c\right) & \text { for } \alpha_{i+}^{n} \leqslant x \cdot \nu_{n} \leqslant \alpha_{(i+1)-}^{n}, \\
\bar{u}\left(x_{1}+\lambda h_{n} x_{3}\right)-\beta_{m}^{n}+\ell \varepsilon_{n}\left(\frac{a}{2} \sqrt{1+\lambda^{2}}+\left((-1)^{m+1}+\kappa_{m}\right) c\right) & \text { for } x \cdot \nu_{n} \geqslant \alpha_{m+}^{n} .\end{cases}
\end{aligned}
$$

Using (6.24)-(6.25) one may check that $u_{n}$ and $\nabla u_{n}$ are continuous across each interface $\left\{x \cdot \nu_{n}=\alpha_{i \pm}^{n}\right\}$, and thus $u_{n} \in H^{2}\left(\Omega ; \mathbb{R}^{3}\right)$. In addition $\partial_{2} u_{n} \equiv 0$, and

$$
\left(\partial_{1} u_{n}, \frac{1}{h_{n}} \partial_{3} u_{n}\right)(x)= \begin{cases}\nabla v\left((-1)^{i+1} \frac{x_{1}-\alpha_{i}}{\ell \varepsilon_{n}},(-1)^{i+1} \frac{h_{n} x_{3}}{\ell \varepsilon_{n}}\right) & \text { for } \alpha_{i-}^{n}<x \cdot \nu_{n}<\alpha_{i+}^{n}, \\ \left(\bar{u}^{\prime}\left(x_{1}+\lambda h_{n} x_{3}\right), \lambda \bar{u}^{\prime}\left(x_{1}+\lambda h_{n} x_{3}\right)\right) & \text { otherwise. }\end{cases}
$$

Then one observes that the maps $x \in \Omega \mapsto \bar{u}\left(x_{1}+\lambda h_{n} x_{3}\right)$ and $x \in \Omega \mapsto$ $\bar{u}^{\prime}\left(x_{1}+\lambda h_{n} x_{3}\right)$ converge to $u$ and $b$ in $W^{1, p}\left(\Omega ; \mathbb{R}^{3}\right)$ and in $L^{p}\left(\Omega ; \mathbb{R}^{3}\right)$ respectively as $n \rightarrow \infty$ (here we also use the fact that $\left.b=\lambda \bar{u}^{\prime}\right)$. On the other hand, $v$ and $\nabla v$ are bounded in $\left\{\left|y \cdot \nu_{\lambda}\right| \leqslant 1 / 2\right\}$ by periodicity in the direction $\nu_{\lambda}^{\perp}$, and $\left|\beta_{i}^{n}\right| \leqslant C \varepsilon_{n}$ for a constant $C$ independent of $i$ and $n$ by the Lipschitz continuity of $\bar{u}$. Hence $u_{n} \rightarrow u$ in $W^{1, p}\left(\Omega ; \mathbb{R}^{3}\right)$ and $\frac{1}{h_{n}} \partial_{3} u_{n} \rightarrow b$ in $L^{p}\left(\Omega ; \mathbb{R}^{3}\right)$.

By (4.31) we have for $n$ large,

$\Omega \cap\left\{\alpha_{i-}^{n}<x \cdot \nu_{n}<\alpha_{i+}^{n}\right\} \subset\left\{x \in \mathbb{R}^{3}: \alpha_{i-}^{n}<x \cdot \nu_{n}<\alpha_{i+}^{n},\left|x_{3}\right|<1 / 2, x_{2} \in J_{i}^{\prime}\right\}=: \Omega_{i}^{n}$,

Using (6.26) we estimate for $n$ large enough,

$$
F_{\varepsilon_{n}}^{h_{n}}\left(u_{n}\right) \leqslant \sum_{i=1}^{m} F_{\varepsilon_{n}}^{h_{n}}\left(\ell \varepsilon_{n} w_{n}^{i}, \Omega_{i}^{n}\right)
$$


and it remains to estimate each term of the sum in the right-hand side of (6.27).

Changing variables, one obtains

$$
F_{\varepsilon_{n}}^{h_{n}}\left(\ell \varepsilon_{n} w_{n}^{i}, \Omega_{i}^{n}\right)=\frac{\ell \varepsilon_{n}}{h_{n}} \mathcal{H}^{1}\left(J_{i}^{\prime}\right) \int_{\Theta_{i}^{n}} \ell \mathcal{W}(\nabla v)+\frac{1}{\ell}\left|\nabla^{2} v\right|^{2} d y,
$$

where $\Theta_{i}^{n}:=\left\{y \in \mathbb{R}^{2}:\left|y \cdot \nu_{\lambda}\right|<1 / 2,\left|y_{2}\right|<h_{n} /\left(2 \ell \varepsilon_{n}\right)\right\}$. Notice that for every $t \in\left(-\frac{1}{2}, \frac{1}{2}\right)$, we have

$$
\begin{aligned}
\Theta_{i}^{n} \cap\left\{y \cdot \nu_{\lambda}=t\right\} & =\left\{y \cdot \nu_{\lambda}=t\right\} \cap\left\{\left|y \cdot \nu_{\lambda}^{\perp}+\lambda t\right|<\frac{h_{n} \sqrt{1+\lambda^{2}}}{2 \ell \varepsilon_{n}}\right\} \\
& \subset\left\{y \cdot \nu_{\lambda}=t\right\} \cap\left\{\left|y \cdot \nu_{\lambda}^{\perp}+\lambda t\right|<\frac{N_{n}}{2}\right\},
\end{aligned}
$$

with $N_{n}:=\left[\frac{h_{n} \sqrt{1+\lambda^{2}}}{\ell \varepsilon_{n}}\right]+1$. Using Fubini's theorem and the periodicity of $v$, we estimate

$$
\begin{aligned}
\int_{\Theta_{i}^{n}} \ell \mathcal{W}(\nabla v)+\frac{1}{\ell}\left|\nabla^{2} v\right|^{2} d y & =\int_{-\frac{1}{2}}^{\frac{1}{2}}\left(\int_{\Theta_{i}^{n} \cap\left\{y \cdot \nu_{\lambda}=t\right\}} \ell \mathcal{W}(\nabla v)+\frac{1}{\ell}\left|\nabla^{2} v\right|^{2} d \mathcal{H}^{1}\right) d t \\
& \leqslant \int_{-\frac{1}{2}}^{\frac{1}{2}}\left(\int_{\left\{y \cdot \nu_{\lambda}=t\right\} \cap\left\{\left|y \cdot \nu_{\lambda}^{\perp}+\lambda t\right|<\frac{N_{n}}{2}\right\}} \ell \mathcal{W}(\nabla v)+\frac{1}{\ell}\left|\nabla^{2} v\right|^{2} d \mathcal{H}^{1}\right) d t \\
& \leqslant N_{n} \int_{Q_{\lambda}^{\prime}} \ell \mathcal{W}(\nabla v)+\frac{1}{\ell}\left|\nabla^{2} v\right|^{2} d y
\end{aligned}
$$

Combining (6.28) with (6.29) yields

$$
F_{\varepsilon_{n}}^{h_{n}}\left(\ell \varepsilon_{n} w_{n}^{i}, \Omega_{i}^{n}\right) \leqslant \frac{\ell \varepsilon_{n} N_{n}}{h_{n} \sqrt{1+\lambda^{2}}}\left(K_{\infty}+2^{-k}\right) \mathcal{H}^{1}\left(J_{i}^{\prime}\right) .
$$

Summing up over $i$ this last inequality, and passing to the limit $n \rightarrow+\infty$ in (6.27) leads to

$$
\limsup _{n \rightarrow \infty} F_{\varepsilon_{n}}^{h_{n}}\left(u_{n}\right) \leqslant K_{\infty} \operatorname{Per}_{\omega}(E)+C_{0} 2^{-k},
$$

for a constant $C_{0}$ independent of $k$. Then the conclusion follows for a suitable diagonal sequence as already pursued in the proof of Theorem 4.11, Step 3.

Remark 6.8. Let us consider an arbitrary sequence $\varepsilon_{n} \rightarrow 0^{+}$and $h>0$ fixed, and assume for simplicity that $W(\xi)=\operatorname{dist}(\xi,\{A, B\})^{p}$. If (1.9) holds, we can apply the results in [16] to infer that the functionals $\left\{\mathbf{E}_{\varepsilon_{n}}\left(\cdot, \Omega_{h}\right)\right\}$ [defined in (1.1)] $\Gamma$-converge for the strong $L^{1}\left(\Omega_{h}\right)$-topology to

$\mathbf{E}_{0}\left(\mathbf{u}, \Omega_{h}\right):= \begin{cases}K_{*} \operatorname{Per}_{\Omega_{h}}(\{\nabla \mathbf{u}=B\}) & \text { if } \mathbf{u} \in W^{1,1}\left(\Omega_{h} ; \mathbb{R}^{3}\right), \text { and } \nabla \mathbf{u} \in B V\left(\Omega_{h} ;\{A, B\}\right), \\ +\infty & \text { otherwise, }\end{cases}$

with $K_{*}=K_{\infty} / \sqrt{1+\lambda^{2}}$. Let us now consider the rescaling $u(x)=$ $\mathbf{u}\left(x_{1}, x_{2}, x_{3} / h\right)$, and define the functional $F_{0}^{h}: L^{1}\left(\Omega ; \mathbb{R}^{3}\right) \rightarrow[0, \infty]$ by

$$
F_{0}^{h}(u):=\frac{1}{h} \mathbf{E}_{0}\left(\mathbf{u}, \Omega_{h}\right) .
$$

By $[6,16]$ (see also Theorem 2.1), if $\mathbf{u}$ has finite energy, then the set $F:=$ $\{\nabla \mathbf{u}=B\}$ is layered perpendicularly to the vector $\nu_{\lambda}$. Setting $E:=F \cap \omega$, we 
easily obtain that $\operatorname{Per}_{\Omega_{h}}(F)=h \sqrt{1+\lambda^{2}} \operatorname{Per}_{\omega}(E)+o(h)$, and thus

$$
F_{0}^{h}(u)=K_{\infty} \operatorname{Per}_{\omega}(E)+o(1) .
$$

It is then straightforward to show that the family $\left\{F_{0}^{h}\right\} \Gamma$-converges for the strong $L^{1}$-topology to $\mathscr{F}_{\infty}$ as $h \rightarrow 0^{+}$.

\subsection{Some rigidity properties}

For $\varepsilon \ll h$ we expect the thin film to behave like a three dimensional sample by separation of scales, so that sequences with uniformly bounded energy should have trivial limits under suitable assumptions on $A$ and $B$. The first situation we consider is when $A^{\prime}=B^{\prime}$ (so $A$ and $B$ are rank-one connected). Indeed, in this case if we first perform the asymptotic $\varepsilon \rightarrow 0$ (see Remark 6.8), the limiting configurations $u$ with finite energy must satisfy $\nabla u=\chi_{K}\left(x_{3}\right) A+(1-$ $\left.\chi_{K}\left(x_{3}\right)\right) B$ for some finite perimeter set $K \subset I$, and the $\Gamma$-limit is proportional to $\frac{1}{h} \operatorname{Card}(\partial K) \mathcal{L}^{2}(\omega)$, see [16]. This latter energy can be bounded with respect to $h$ only if $\operatorname{Card}(\partial K)=0$ for $h$ small, and it formally explain the expected rigidity effect. We have rigorously proved this fact only in the case where $\varepsilon$ is sufficiently small relative to $h$ as stated in the following theorem.

Theorem 6.9. Assume $\left(H_{1}\right)-\left(H_{3}\right)$ and $(1.5)$ hold with $A^{\prime}=B^{\prime}$. Let $h_{n} \rightarrow 0^{+}$ and $\varepsilon_{n} \rightarrow 0^{+}$be arbitrary sequences such that $\sup _{n} \varepsilon_{n} / h_{n}^{p}<\infty$. Then, for any $\left\{u_{n}\right\} \subset H^{2}\left(\Omega ; \mathbb{R}^{3}\right)$ such that $\sup _{n} F_{\varepsilon_{n}}^{h_{n}}\left(u_{n}\right)<\infty$, there exist a subsequence (not relabeled) and $\xi_{0} \in\{A, B\}$ such that $\nabla_{h_{n}} u_{n} \rightarrow \xi_{0}$ in $L^{p}\left(\Omega ; \mathbb{R}^{3 \times 3}\right)$.

Proof. By Theorem 1.1, we can find a subsequence such that $\nabla_{h_{n}} u \rightarrow\left(\nabla^{\prime} u, b\right)$ in $L^{p}\left(\Omega ; \mathbb{R}^{3 \times 3}\right)$ for some $(u, b) \in \mathscr{C}$. Since $A^{\prime}=B^{\prime}, \nabla^{\prime} u$ is constant, and we only have to prove that $b$ is constant. By (1.5) we have $A^{\prime}=B^{\prime}=0$, and thus Lemma 4.8 yields

$$
W(\xi) \geqslant \frac{1}{C_{*}}\left(\left|\xi^{\prime}\right|^{p}+\min \left\{\left|\xi_{3}-A_{3}\right|^{p},\left|\xi_{3}-B_{3}\right|^{p}\right\}\right), \quad \forall \xi \in \mathbb{R}^{3 \times 3},
$$

Setting $v_{n}:=\frac{1}{h_{n}} u_{n}$, we deduce that

$$
\sup _{n \in \mathbb{N}} \int_{\Omega} \frac{h_{n}^{p}}{\varepsilon_{n}}\left|\nabla^{\prime} v_{n}\right|^{p}+\frac{1}{\varepsilon_{n}} \min \left\{\left|\partial_{3} v_{n}-A_{3}\right|^{p},\left|\partial_{3} v_{n}-B_{3}\right|^{p}\right\} d x<\infty .
$$

Hence $\left\{\nabla v_{n}\right\}$ is bounded in $L^{p}\left(\Omega ; \mathbb{R}^{3}\right)$. By Poincaré's inequality, there exists a further subsequence (not relabeled) such that $v_{n}-f_{\Omega} v_{n} \rightarrow v$ weakly in $W^{1, p}\left(\Omega ; \mathbb{R}^{3}\right)$ for some $v \in W^{1, p}\left(\Omega ; \mathbb{R}^{3}\right)$. But since $\partial_{3} v_{n}=\frac{1}{h_{n}} \partial u_{n}$, we have $\partial_{3} v_{n} \rightarrow b$ strongly in $L^{p}\left(\Omega ; \mathbb{R}^{3}\right)$. Hence $\partial_{3}\left(v(x)-b\left(x^{\prime}\right) x_{3}\right)=0$, and we can argue as in Theorem 2.1, Step 2, to prove that $v(x)=b\left(x^{\prime}\right) x_{3}+w\left(x^{\prime}\right)$ for some function $w \in B V\left(\omega ; \mathbb{R}^{3}\right)$. Integrating this equality in $x_{3}$ over the interval $I$ yields $w\left(x^{\prime}\right)=\int_{I} v\left(x^{\prime}, x_{3}\right) d x_{3}$ a.e. in $\omega$. It obviously implies that $w \in W^{1, p}\left(\omega ; \mathbb{R}^{3}\right)$. Since $b\left(x^{\prime}\right) x_{3}=v(x)-w\left(x^{\prime}\right)$, we conclude that $b \in W^{1, p}\left(\omega ;\left\{A_{3}, B_{3}\right\}\right)$, and thus $b$ must be constant.

The other case where one can expect rigidity is when $A$ and $B$ are not rank-one connected, and thus not compatible in the bulk [6]. We will show that rigidity occurs at least for some particular potentials $W$ as a consequence 
of a two-wells rigidity estimate due to Chaudhuri and Müller [14] (see [21] for single well rigidity). The class of double-well potentials we consider is as follows. For simplicity we will assume that

$$
A=I_{d}, \quad \text { and } \quad B=\operatorname{diag}\left(\theta_{1}, 1, \theta_{2}\right),
$$

for some $\theta_{1}, \theta_{2} \in \mathbb{R}$ satisfying

$$
\theta_{i}>0 \quad i=1,2, \quad \text { and } \quad\left(1-\theta_{1}\right)\left(1-\theta_{2}\right)>0 .
$$

Here $I_{d}$ denotes the $3 \times 3$ identity matrix. The second assumption in (6.31) corresponds to the strong incompatibility condition between $A$ and $B$ in the sense of Matos [29] (see also [14,15]). Noticing that $A^{\prime}$ and $B^{\prime}$ are rank-one connected, we shall consider continuous potentials $W: \mathbb{R}^{3 \times 3} \rightarrow[0, \infty)$ such that $\left(H_{1}\right)-\left(H_{3}\right)$ hold with $p=2$.

Using the rigidity estimate of [14] and an argument similar to [15], we have obtained the following result.

Theorem 6.10. Assume $\left(H_{1}\right)-\left(H_{3}\right)$ hold with $p=2$, (6.30), and (6.31). Let $h_{n} \rightarrow 0^{+}$and $\varepsilon_{n} \rightarrow 0^{+}$be arbitrary sequences such that $\varepsilon_{n} / h_{n} \rightarrow \infty$. Then, for any sequence $\left\{u_{n}\right\} \subset H^{2}\left(\Omega ; \mathbb{R}^{3}\right)$ such that $\sup _{n} F_{\varepsilon_{n}}^{h_{n}}\left(u_{n}\right)<\infty$, there exist a subsequence (not relabeled) and $\xi_{0} \in\{A, B\}$ such that $\nabla_{h_{n}} u_{n} \rightarrow \xi_{0}$ in $L^{2}\left(\Omega ; \mathbb{R}^{3 \times 3}\right)$.

Proof. By Theorem 1.1, there is a subsequence such that $u_{n}-f_{\Omega} u_{n} d x \rightarrow u$ in $H^{1}\left(\Omega ; \mathbb{R}^{3}\right)$ and $\frac{1}{h_{n}} \partial_{3} u_{n} \rightarrow b$ in $L^{2}\left(\Omega ; \mathbb{R}^{3}\right)$ for some $(u, b) \in \mathscr{C}$. To prove the announced result, it suffices to prove that for an arbitrary open set $\mathcal{O} \subset$ $\omega,\left(\nabla^{\prime} u, b\right)$ is constant in $\mathcal{O} \times I$. Without loss of generality we may assume that $\mathcal{O}=Q^{\prime}$ the unit cube of $\mathbb{R}^{2}$. We proceed as follows.

Step 1. First we infer from Lemma 4.8 that

$$
\begin{aligned}
& W(\xi) \geqslant \frac{1}{C_{*}} \min \left\{\min _{R \in S O(3)}|\xi-R|^{2}, \min _{R \in S O(3)}|\xi-R B|^{2}\right\} \\
& =\frac{1}{C_{*}} \operatorname{dist}^{2}(\xi, K) \quad \forall \xi \in \mathbb{R}^{3 \times 3},
\end{aligned}
$$

where $K:=S O(3) \cup S O(3) B$. From this estimate we deduce that

$$
\frac{1}{h_{n}} \int_{Q} \operatorname{dist}^{2}\left(\nabla_{h_{n}} u_{n}, K\right) d x \leqslant C \frac{\varepsilon_{n}}{h_{n}} .
$$

Setting $M_{n}:=\left[\frac{2}{h_{n}}\right]$, we now divide $Q^{\prime}$ into $M_{n}^{2}$ squares $S_{a, n}$ of the form

$$
S_{a, n}=a+M_{n}^{-1} Q^{\prime} \quad \text { with } \quad a \in \mathscr{A}^{n}:=M_{n}^{-1} \mathbb{Z}^{2} \cap Q^{\prime},
$$

so that $Q^{\prime}=\cup_{a \in \mathscr{A}^{n}} S_{a, n}$ up to a set of $\mathcal{L}^{2}$-measure zero. Then for each $a \in \mathscr{A}^{n}$, we define the rescaled map $v_{n}^{a}: M_{n}^{-1} Q \rightarrow \mathbb{R}^{3}$ by $v_{n}^{a}(y):=u_{n}\left(a+y^{\prime}, \frac{y_{3}}{h_{n}}\right)$. By [14, Theorem 2], there exists a universal constant $C_{\text {univ }}$ such that for each $a \in \mathscr{A}^{n}$ we can find $R_{n}^{a} \in K$ satisfying

$$
\int_{M_{n}^{-1} Q}\left|\nabla v_{n}^{a}-R_{n}^{a}\right|^{2} d y \leqslant C_{\text {univ }} \int_{M_{n}^{-1} Q} \operatorname{dist}^{2}\left(\nabla v_{n}^{a}, K\right) d y .
$$


Scaling back, we derive that

$$
\int_{S_{a, n} \times \frac{1}{2} I}\left|\nabla_{h_{n}} u_{n}-R_{n}^{a}\right|^{2} d x \leqslant C_{\text {univ }} \int_{S_{a, n} \times I} \operatorname{dist}^{2}\left(\nabla_{h_{n}} u_{n}, K\right) d x \quad \forall a \in \mathscr{A}^{n} .
$$

Defining the piecewise constant map $R_{n}: Q^{\prime} \rightarrow K$ by $R_{n}\left(x^{\prime}\right):=R_{n}^{a}$ for $x^{\prime} \in S_{a, n}$, and adding the previous inequalities in (6.33) leads to

$$
\int_{Q^{\prime} \times \frac{1}{2} I}\left|\nabla_{h_{n}} u_{n}-R_{n}\left(x^{\prime}\right)\right|^{2} d x \leqslant C_{\text {univ }} \int_{Q} \operatorname{dist}^{2}\left(\nabla_{h_{n}} u_{n}, K\right) d x \leqslant C \varepsilon_{n} \underset{n \rightarrow \infty}{\longrightarrow} 0,
$$

thanks to $(6.32)$. Since $\nabla_{h_{n}} u_{n} \rightarrow\left(\nabla^{\prime} u, b\right)$ in $L^{2}\left(\Omega ; \mathbb{R}^{3 \times 3}\right)$, we conclude that $R_{n} \rightarrow\left(\nabla^{\prime} u, b\right)$ in $L^{2}\left(Q^{\prime} ; \mathbb{R}^{3 \times 3}\right)$.

Step 2. Let $\delta>0$ be a small parameter to be chosen. We divide $\mathscr{A}^{n}$ into the following classes,

$$
\mathscr{A}_{0}^{n}:=\left\{a \in \mathscr{A}^{n}: \int_{S_{a, n} \times I} \operatorname{dist}^{2}\left(\nabla_{h_{n}} u_{n}, K\right) d x \geqslant \delta h_{n}^{2}\right\},
$$

$\mathscr{A}_{1}^{n}:=\left\{a \in \mathscr{A}^{n} \backslash \mathscr{A}_{0}^{n}: R_{n}^{a} \in S O(3)\right\}$, and $\mathscr{A}_{2}^{n}:=\left\{a \in \mathscr{A}^{n} \backslash \mathscr{A}_{0}^{n}: R_{n}^{a} \in\right.$ $S O(3) B\}$. We observe that (6.32) yields

$$
\operatorname{Card}\left(\mathscr{A}_{0}^{n}\right) \leqslant \frac{1}{\delta h_{n}^{2}} \int_{Q} \operatorname{dist}^{2}\left(\nabla_{h_{n}} u_{n}, K\right) d x=o\left(1 / h_{n}\right),
$$

where Card denotes the counting measure. Next we consider the sets

$$
G_{0}^{n}:=\bigcup_{a \in \mathscr{A}_{0}^{n}} S_{a, n}, \quad G_{1}^{n}:=\bigcup_{a \in \mathscr{A}_{1}^{n}} S_{a, n}, \quad G_{2}^{n}:=\bigcup_{a \in \mathscr{A}_{2}^{n}} S_{a, n},
$$

so that $Q^{\prime}=G_{0}^{n} \cup G_{1}^{n} \cup G_{2}^{n}$ up to a set of $\mathcal{L}^{2}$-measure zero.

Now, we shall enumerate the edges $\Gamma_{a}^{j}(j=1,2,3,4)$ of a square $S_{a, n}$ according the counterclockwise sense, $\Gamma_{a}^{1}$ being the bottom edge. We observe that each boundary $\partial G_{i}^{n}$ is polyhedral and made by the edges $\Gamma_{a}^{j}$ (of length $M_{n}^{-1}$ ) of some squares $S_{a, n}$ with $a \in \mathscr{A}_{i}^{n}$, that we call boundary squares. For $i=0,1,2$ and $j=1,2,3,4$, we set

$\mathscr{B}_{i}^{n}:=\left\{a \in \mathscr{A}_{i}^{n}: S_{a, n}\right.$ is a boundary square $\} \quad$ and $\mathscr{E}_{i, j}^{n}:=\left\{a \in \mathscr{B}_{i}^{n}: \Gamma_{a}^{j} \subset \partial G_{i}^{n} \cap Q^{\prime}\right\}$.

We claim that if $\delta>0$ is chosen small enough, then for every $n \in \mathbb{N}$ large enough, and for $i=1,2, j=1,2,3,4$,

$$
\Gamma_{a}^{j} \subset \partial G_{0}^{n} \quad \forall a \in \mathscr{E}_{i, j}^{n} .
$$

We shall prove (6.35) in the next step. Assuming that (6.35) is true, we estimate for $i=1,2$,

$$
\mathcal{H}^{1}\left(\partial G_{i}^{n} \cap Q^{\prime}\right)=\sum_{j=1}^{4} \sum_{a \in \mathscr{E}_{i, j}^{n}} \mathcal{H}^{1}\left(\Gamma_{a}^{j}\right) \leqslant 4 h_{n} \operatorname{Card}\left(\mathscr{A}_{0}^{n}\right) \underset{n \rightarrow \infty}{\longrightarrow} 0,
$$

thanks to (6.34). Therefore, we can extract a subsequence such that for $i=1,2$, either $\mathcal{L}^{2}\left(Q^{\prime} \backslash G_{i}^{n}\right) \rightarrow 0$ or $\mathcal{L}^{2}\left(G_{i}^{n}\right) \rightarrow 0$. Since $\mathcal{L}^{2}\left(G_{0}^{n}\right) \rightarrow 0$ by (6.34), and $Q^{\prime}=G_{0}^{n} \cup G_{1}^{n} \cup G_{2}^{n}$, we must have $\mathcal{L}^{2}\left(Q^{\prime} \backslash G_{1}^{n}\right) \rightarrow 0$ or $\mathcal{L}^{2}\left(Q^{\prime} \backslash G_{2}^{n}\right) \rightarrow 0$. 
Without loss of generality, we may assume that $\mathcal{L}^{2}\left(Q^{\prime} \backslash G_{1}^{n}\right) \rightarrow 0$. Then we estimate

$\int_{Q^{\prime}} \operatorname{dist}^{2}\left(\left(\nabla^{\prime} u, b\right), S O(3)\right) d x^{\prime} \leqslant \int_{Q^{\prime}}\left|\left(\nabla^{\prime} u, b\right)-R_{n}\right|^{2} d x+C \mathcal{L}^{2}\left(Q^{\prime} \backslash G_{1}^{n}\right) \underset{n \rightarrow \infty}{\longrightarrow} 0$,

which yields $\left(\nabla^{\prime} u, b\right)\left(x^{\prime}\right) \in S O(3) \cap\left\{I_{d}, B\right\}$ for $\mathcal{L}^{2}$-a.e. $x^{\prime} \in Q^{\prime}$. Since $B \notin$ $S O(3)$, we finally conclude that $\left(\nabla^{\prime} u, b\right) \equiv I_{d}$ in $Q^{\prime}$.

Step 3. It remains to prove (6.35). We argue by contradiction. Without loss of generality, we may assume that there exists $a \in \mathscr{E}_{1,1}$ such that $\Gamma_{a}^{1} \not \subset \partial G_{0}^{n}$. Since $\Gamma_{a}^{1} \subset Q^{\prime}$, we have $\tilde{a}:=a-\left(0, M_{n}^{-1}\right) \in M_{n}^{-1} \mathbb{Z}^{2} \cap Q^{\prime}$, and $\tilde{a} \notin \mathscr{A}_{0}^{n} \cup \mathscr{A}_{1}^{n}$. Thus $\tilde{a} \in \mathscr{A}_{2}^{n}$. As in Step 1, we can apply [14, Theorem 2] to find $\tilde{R}_{n}^{a} \in K$ such that

$$
\begin{aligned}
\frac{1}{h_{n}^{2}} \int_{\left(S_{a, n} \cup S_{\tilde{a}, n}\right) \times \frac{1}{2} I}\left|\nabla_{h_{n}} u_{n}-\tilde{R}_{n}^{a}\right|^{2} d x & \leqslant \frac{\tilde{C}_{\text {univ }}}{h_{n}^{2}} \int_{\left(S_{a, n} \cup S_{\tilde{a}, n}\right) \times I} \operatorname{dist}^{2}\left(\nabla_{h_{n}} u_{n}, K\right) d x \\
& \leqslant 2 \max \left\{C_{\text {univ }}, \tilde{C}_{\text {univ }}\right\} \delta,
\end{aligned}
$$

for some universal constant $\tilde{C}_{\text {univ }}$. Then we have

$\left|R_{n}^{a}-\tilde{R}_{n}^{a}\right|^{2} \leqslant \frac{16}{h_{n}^{2}} \int_{\left(S_{a, n}\right) \times \frac{1}{2} I}\left|\nabla_{h_{n}} u_{n}-R_{n}^{a}\right|^{2}+\left|\nabla_{h_{n}} u_{n}-\tilde{R}_{n}^{a}\right|^{2} d x \leqslant 32 \max \left\{C_{\text {univ }}, \tilde{C}_{\text {univ }}\right\} \delta$.

We proceed similarly to get $\left|R_{n}^{\tilde{a}}-\tilde{R}_{n}^{a}\right|^{2} \leqslant 32 \max \left\{C_{\text {univ }}, \tilde{C}_{\text {univ }}\right\} \delta$, and we obtain a contradiction whenever $\delta<\left[32 \max \left\{C_{\text {univ }}, \tilde{C}_{\text {univ }}\right\}\right]^{-1} \operatorname{dist}^{2}(S O(3), S O(3) B)$.

\section{Acknowledgments}

The authors thank J.-F. Babadjian, I. Fonseca, and G. Leoni for their advices during the preparation of this work. Part of this work was done while B. Galvão-Sousa was postdoctoral fellow at McMaster University, and it was partially supported by Fundação para a Ciência e a Tecnologia under grant PRAXIS XXI SFRH/BD/8582/2002, and by the Center for Nonlinear Analysis (CNA) under the National Science Fundation Grant No. 0405343. The research of $\mathrm{V}$. Millot was partially supported by the Agence Nationale de la Recherche under Grant ANR-10-JCJC 0106.

\section{References}

[1] Ambrosio, L.: Metric space valued functions of bounded variations. Ann. Scuola Norm. Sup. Pisa Cl. Sci. 17(4), 439-478 (1990)

[2] Ambrosio, L., Dal Maso, G.: On the relaxation in $B V\left(\Omega ; \mathbb{R}^{m}\right)$ of quasiconvex integrals. J. Funct. Anal. 109, 76-97 (1992)

[3] Ambrosio, L., Fonseca, I., Marcellini, P., Tartar, L.: On a volume constrained variational problem. Arch. Ration. Mech. Anal. 149, 23-47 (1999) 
[4] Ambrosio, L., Fusco, N., Pallara, D.: Functions of Bounded Variation and Free Discontinuity Problems, Oxford University Press, New York (2000)

[5] Baldo, S.: Minimal interface criterion for phase transitions in mixtures of CahnHilliard fluids. Ann. Inst. H. Poincaré Anal. Non Linéaire 7, 67-90 (1990)

[6] Ball, J., James, R.D.: Fine phase mixtures as minimizers of energy. Arch. Rational Mech. Anal. 100, 13-52 (1997)

[7] Barroso, A.C., Fonseca, I.: Anisotropic singular perturbations - the vectorial case. Proc. R. Soc. Edinb. Sect. A 124, 527-571 (1994)

[8] Bělík, P., Luskin, M.: The $\Gamma$-convergence of a sharp interface thin film model with nonconvex elastic energy. SIAM J. Math. Anal. 38, 414-433 (2006)

[9] Bhattacharya, K., James, R.D.: A theory of thin films of martensitic materials with applications to microactuators. J. Mech. Phys. Solids 47, 531-576 (1999)

[10] Bouchitté, G.: Singular perturbations of variational problems arising from a two-phase transition model. Appl. Math. Optim. 21, 289-314 (1990)

[11] Bouchitté, G., Fonseca, I., Mascarenhas, M.L.: Bending moment in membrane theory. J. Elast. 73, 75-99 (2004)

[12] Braides, A., Defranceschi, A.: Homogeneization of Multiple Integrals. Claredon Press, Oxford (1998)

[13] Braides, A., Fonseca, I., Francfort, G.: 3D-2D asymptotic analysis for inhomogeneous thin films. Indiana Univ. Math. J. 49, 1367-1404 (2000)

[14] Chaudhuri, N., Müller, S.: Rigidity estimate for two incompatible wells. Calc. Var. Partial Differ. Equ. 19, 379-390 (2004)

[15] Chaudhuri, N., Müller, S.: Scaling of the energy for thin martensitic films. SIAM J. Math. Anal. 38, 468-477 (2006)

[16] Conti, S., Fonseca, I., Leoni, G.: A $\Gamma$-convergence result for the two-gradient theory of phase transitions. Comm. Pure Appl. Math. 55, 857-936 (2002)

[17] Conti, S., Schweizer, B.: Rigidity and Gamma convergence for solid-solid phase transitions with $S O(2)$-invariance. Comm. Pure Appl. Math. 59, 830-868 (2006)

[18] Conti, S., Schweizer, B.: A sharp interface limit for a two-well problem in geometrically linear elasticity. Arch. Ration. Mech. Anal. 179, 413-452 (2006)

[19] Dal Maso, G.: An Introduction to Г-Convergence. Birkhaüser, Boston (1993)

[20] Fonseca, I., Francfort, G., Leoni, G.: Thin elastic films: the impact of higher order perturbations, Quart. Appl. Math. 65, 69-98 (2007); Erratum, Quart. Appl. Math. 66, 781-799 (2008)

[21] Friesecke, G., James, R. D., Müller, S.: A theorem on geometric rigidity and the derivation of nonlinear plate theory from three dimensional elasticity. Comm. Pure Appl. Math. 55, 1461-1506 (2002) 
[22] Friesecke, G., James, R.D., Müller, S.: A hierarchy of plate models derived from nonlinear elasticity by Gamma-convergence. Arch. Rational Mech. Anal. 180, 183-236 (2006)

[23] Fonseca, I., Mantegazza, C.: Second order singular perturbation models for phase transitions. SIAM J. Math. Anal. 31, 1121-1143 (2000)

[24] Fonseca, I., Tartar, L.: The gradient theory of phase transitions for systems with two potential wells. Proc. R. Soc. Edinb. Sect. A 111, 89-102 (1989)

[25] Gurtin, M.E.: On a theory of phase transitions with interfacial energy. Arch. Rational Mech. Anal. 87, 187-212 (1984)

[26] Hornung, P.: A Gamma-convergence result for thin martensitic films in linearized elasticity. SIAM J. Math. Anal. 40, 186-214 (2008)

[27] Jin, W., Kohn, R.V.: Singular perturbation and the energy of folds. J. Nonlinear Sci. 10, 355-390 (2000)

[28] Le Dret, H., Raoult, A.: The nonlinear membrane model as variational limit of nonlinear three dimensional elasticity. J. Math. Pures Appl. 74, 549-578 (1995)

[29] Matos, J.: Young measures and the absence of fine microstructures in a class of phase transitions. Eur. J. Appl. Math. 3, 31-54 (1992)

[30] Modica, L.: The gradient theory of phase transitions and the minimal interface criterion. Arch. Ration. Mech. Anal. 98, 123-142 (1987)

[31] Modica, L., Mortola, S. Un esempio di $\Gamma$-convergenza. Boll. Un. Mat. Ital. B 14(5), 285-299 (1977)

[32] Owen, N., Sternberg, P.: Nonconvex variational problems with anisotropic perturbations. Nonlinear Anal. 16, 531-559 (1991)

[33] Pedregal, P.: Parametrized measures and variational principles, Progress in Nonlinear Differential Equations and their Applications 30. Birkhäuser Verlag, Basel (1997)

[34] Shu, Y.C.: Heterogeneous thin films of martensitic materials. Arch. Rat. Mech. Anal. 153, 39-90 (2000)

[35] Sternberg, P.: The effect of a singular perturbation on nonconvex variational problems. Arch. Rational Mech. Anal. 101, 209-260 (1988)

Bernardo Galvão-Sousa

Department of Mathematics

University of Toronto

Toronto

$\mathrm{ON}$

Canada

e-mail: beni@math.toronto.edu 
Vincent Millot

Université Paris Diderot - Paris 7

UMR 7598 Laboratoire J.-L. Lions

Paris

France

e-mail: millot@ljll.univ-paris-diderot.fr

Received: 29 August 2012.

Accepted: 7 February 2013. 